\title{
ON UNITARY UNIPOTENT REPRESENTATIONS OF $p$-ADIC GROUPS AND AFFINE HECKE ALGEBRAS WITH UNEQUAL PARAMETERS
}

\author{
DAN CIUBOTARU
}

\begin{abstract}
We determine the unitary dual of the geometric graded Hecke algebras with unequal parameters which appear in Lusztig's classification of unipotent representations for exceptional $p$-adic groups. The largest such algebra is of type $F_{4}$. Via the Barbasch-Moy correspondence of unitarity applied to this setting, this is equivalent to the identification of the corresponding unitary unipotent representations with real central character of the $p$-adic groups. In order for this correspondence to be applicable here, we show (following Lusztig's geometric classification, and Barbasch and Moy's original argument) that the set of tempered modules with real central character for a geometric graded Hecke algebra is linearly independent when restricted to the Weyl group.
\end{abstract}

\section{INTRODUCTION}

1.1. The category of representations with unipotent cuspidal support for the rational forms inner to a split $p$-adic group of adjoint type is equivalent to a direct product of categories of affine Hecke algebras with unequal parameters (Lu6]). We are interested in the problem of identifying the unitary representations in this category.

Let us briefly recall first the Deligne-Langlands-Lusztig correspondence first. Let $\mathbb{F}$ denote a finite extension of the $p$-adic numbers, with residue field $\mathbb{F}_{q}$. Let $\mathcal{G}$ denote a connected adjoint quasi-simple algebraic group defined over the algebraic completion $\overline{\mathbb{F}}$. Let $\Psi_{s}$ be the inner class of $\mathcal{G}$ which contains the split $\mathbb{F}$-form of $\mathcal{G}$. For every $\mathcal{G}(\mathbb{F}) \in \Psi_{s}$, one defines the category of unipotent representations Uni $(\mathcal{G}(\mathbb{F})$ ) as follows. For every parahoric subgroup $J$ of $\mathcal{G}(\mathbb{F})$, let $\bar{J}$ denote the reductive quotient (a reductive group over $\mathbb{F}_{q}$ ). For every unipotent cuspidal representation $\bar{\rho}$ of $\bar{J}$ (in the sense of Deligne-Lusztig), let $\rho$ be the representation obtained by pulling back to $J$. (The unipotent cuspidal representations appear very rarely.) Then let $\operatorname{Uni}_{(J, \rho)}(\mathcal{G}(\mathbb{F}))$ denote the category of admissible representations $\pi$ of $\mathcal{G}(\mathbb{F})$ such that $\operatorname{Hom}_{J}[\pi: \rho]$ is nonzero and generates $\pi$. Set Uni $(\mathcal{G}(\mathbb{F}))=\bigsqcup_{(J, \rho)} \operatorname{Uni}_{(J, \rho)}(\mathcal{G}(\mathbb{F}))$, and $\operatorname{Uni}(\mathcal{G})=\bigsqcup_{\mathcal{G}(\mathbb{F}) \in \Psi_{s}} \operatorname{Uni}(\mathcal{G}(\mathbb{F}))$. This is the "arithmetic side" of the correspondence.

For every $(J, \rho)$ that appears, set $\mathcal{H}(\mathcal{G}(\mathbb{F}), J, \rho)=\operatorname{End}\left(\operatorname{Ind}_{J}^{\mathcal{G}(\mathbb{F})}(\rho)\right)$. This is an affine Hecke algebra with unequal parameters. The prototype is the subcategory of representations generated by their vectors fixed under an Iwahori subgroup, that is, Uni $(\mathcal{G}(\mathbb{F}), I$, triv $)$, where $I$ is an Iwahori subgroup. The irreducible objects in this

Received by the editors January 31, 2007.

2000 Mathematics Subject Classification. Primary 22E50.

(C)2008 American Mathematical Society 453 
category are precisely the subquotients of the unramified principal series of $\mathcal{G}(\mathbb{F})$. By the fundamental results of Borel and Casselman, there is an equivalence of categories between $\operatorname{Uni}(\mathcal{G}(\mathbb{F}), I$, triv $)$ and $\mathcal{H}(\mathcal{G}(\mathbb{F}), I$, triv $)$-mod given by the functor $\pi \mapsto \pi^{I}:=\operatorname{Hom}_{I}[\pi:$ triv $]$. In general, by the results of Morris and Moy-Prasad, there is an equivalence of categories between $\operatorname{Uni}_{(J, \rho)}(\mathcal{G}(\mathbb{F}))$ and $\mathcal{H}(\mathcal{G}(\mathbb{F}), J, \rho)$-mod given by $\pi \mapsto \operatorname{Hom}_{J}[\pi: \rho]$.

On the "geometric side", one considers the complex simply-connected group $G$ with root datum dual to $\mathcal{G}$. Kazhdan-Lusztig in the case of equal parameters, and Lusztig in general, gave a geometric construction of affine Hecke algebras, respectively affine graded Hecke algebras starting from $G$. Let $L$ be a quasi-Levi subgroup of $G$ (i.e, the centralizer of a semisimple element in $G$ ), with Lie algebra $\mathfrak{l}, \mathcal{C}$ a nilpotent class in $\mathfrak{l}$ which admits a cuspidal local system $\mathcal{L}$. (These are very rare.) Then one attaches an affine Hecke algebra $\mathcal{H}(G, L, \mathcal{C}, \mathcal{L})$ with unequal parameters, whose representation theory is classified in terms of perverse sheaves on certain graded nilpotent orbits of $\mathfrak{g}$. The truly remarkable realization of [Lu6] is that the two collections of affine Hecke algebras $\left\{\mathcal{H}(\mathcal{G}(\mathbb{F}), J, \rho): G(\mathbb{F}) \in \Psi_{s},(J, \rho)\right\}$ and $\{\mathcal{H}(G, L, \mathcal{C}, \mathcal{L}):(L, \mathcal{C}, \mathcal{L})\}$ coincide, and via this identification, one translates the geometric classification to $\operatorname{Uni}(\mathcal{G})$.

Theorem ([L6]). There is a one-to-one correspondence between $\operatorname{Uni}(\mathcal{G})$ and $G$ conjugacy classes in

$$
\{(s, e, \psi): s \in G \text { semisimple, } e \in \mathfrak{g}, \operatorname{Ad}(s) e=q e, \psi \in \widehat{A(s, e)}\} .
$$

Note that $e \in \mathfrak{g}$ is necessarily nilpotent. In (1.1.1), $A(s, e)$ denotes the group of components of the centralizer in $G$ of $s$ and $e$.

Remarks. a) We emphasize that the left-hand (arithmetic) side of the correspondence is a union over all rational forms of $\mathcal{G}$ inner to the split one.

b) An individual piece $\operatorname{Uni}(\mathcal{G}(\mathbb{F}))$, corresponding to a fixed rational form, is distinguished by the action of $\psi \in \widehat{A(s, e)}$ on the center $Z(G)$. For example, the split form $G(\mathbb{F})^{s}$ is parameterized by those $\psi$ such that $\left.\psi\right|_{Z(G)}=$ triv. The Iwahori-spherical subcategory $\operatorname{Iw}\left(\mathcal{G}(\mathbb{F})^{s}\right)$ of the split form is parameterized by the representations $\psi$ of Springer type, i.e., those which appear in the natural action of $A(s, e)$ on the Borel-Moore homology of the variety $\mathcal{B}_{s, e}$ of Borel subgroups of $G$ containing both $s$ and $e$ ([KL $)$. The trivial $A(s, e)$-representation is of Springer type.

c) By Re2 (also BM4 in the Iwahori-spherical case), the representations in Uni $\left(\mathcal{G}(\mathbb{F})^{s}\right)$ which are generic in the sense that they admit Whittaker models, are parameterized in theorem 1.1 by $(s, e, \psi)$, where $e$ is in the unique open orbit of $Z_{G}(s)$ on $\mathfrak{g}_{q}:=\{x \in \mathfrak{g}: A d(s) x=q s\}$, and $\psi$ is trivial. Here $Z_{G}(s)$ denotes the centralizer of $s$ in $G$. In particular, these representations are all Iwahori-spherical.

d) The $\left(K\right.$-)spherical representations of $\mathcal{G}(\mathbb{F})^{s}$ correspond to $(s, e, \psi)$ with $e=0$ and $\psi=$ triv. They are Iwahori-spherical too. The Iwahori-Matsumoto involution interchanges spherical and generic representations and by [BM1, BM2], it preserves unitarity.

e) A first calculation we present (in section 4) as an example concerns unitary representations for $\mathcal{G}=E_{6}$. The dual $G$ is also type $E_{6}$, simply connected and $Z(G)=\mathbb{Z} / 3$. Let $E_{6}^{n q s}$ denote any one of the two nonquasisplit form of type $E_{6}$ (denoted ${ }^{3} E_{6}$ in the tables of [Ti]). On the arithmetic side, $\operatorname{Iw}\left(E_{6}^{n q s}\right)$ is the category of Iwahori-spherical representations of $E_{6}^{n q s}$. On the geometric side, by [Lu5], there 
exist two cuspidal local systems for the Levi $L=2 A_{2}$ in $E_{6}$ (notation as in Ca ), and the geometric Hecke algebras are isomorphic. The two cuspidal local systems (actually representations of the component group) are distinguished by how they act on $Z(G)$. By [u6], the geometric Hecke algebra and the Iwahori-Hecke algebra are identical, of type $G_{2}$ with certain unequal parameters (see the tables in [Ti]). At the level of geometric parameters (1.1.1), the component group representations $\psi$ which appear, act on $Z(G)$ like a fixed character of $\mathbb{Z} / 3$ of order 3 .

f) The hardest calculation in this paper refers to the case of unitary representations for $\mathcal{G}=E_{7}$. The dual $G$ is also of type $E_{7}$, simply connected. Then $Z(G)=\mathbb{Z} / 2$, and the two representations of $Z(G)$ correspond to the two rational forms in the split inner class. Let us denote by $E_{7}^{n s}$ the nonsplit form. On the arithmetic side, $\operatorname{Iw}\left(E_{7}^{n s}\right)$ is the category of Iwahori-spherical representations of $E_{7}^{n s}$. The corresponding Iwahori-Hecke algebra is of type $F_{4}$ with certain unequal parameters On the geometric side, there exists a cuspidal local system for the Levi $L=\left(3 A_{1}\right)^{\prime \prime}$ in $E_{7}$. The affine Hecke algebra constructed from it has the same presentation with generators and relations as the Iwahori-Hecke one for $E_{7}^{n s}$. At the level of geometric parameters (1.1.1), the component group representations $\psi$ which are nontrivial on $Z(G)$ appear. The nilpotent orbits of $E_{7}$ which allow such representations are in Figure 2,

g) By changing short roots with long roots in an affine Hecke algebra which is not simply-laced, and scaling the unequal parameters accordingly, one obtains isomorphic Hecke algebras. Using this isomorphism, the same Hecke algebra as in e) controls two subcategories of unipotent representations for $\mathcal{G}(\mathbb{F})=E_{8}^{s}$ (split) and a parahoric of type $E_{6}$, while the Hecke algebra in f) controls a subcategory for $\mathcal{G}(\mathbb{F})=E_{8}^{s}$ and a parahoric of type $D_{4}$. The weight structure of the discrete series modules for these latter Hecke algebras, and their formal degrees, were calculated in Re1. We verified that the dimensions of the discrete series, which we obtained by finding their $W$-structure, agree with the dimensions listed in Re1. Note also that by the Barbasch-Moy transfer of unitarity (see section 1.2) one can obtain interesting correspondences between unitary representations whenever two categories of unipotent representations are controlled by the same affine Hecke algebra.

1.2. The Iwahori-Hecke algebra is a convolution algebra of complex-valued functions, and therefore has a natural *-operation: $f \mapsto f^{*}, f^{*}(g):=\overline{f\left(g^{-1}\right)}$. One defines hermitian and unitary modules with respect to this $*$-operation. The approach to the determination of Iwahori-spherical unitary representations of a split $p$-adic group via a reduction to Iwahori-Hecke algebra modules with real central ("infinitesimal") character was introduced in BM1, BM2]. It is based on the geometric classification, and the use of the graded Hecke algebra of [Lu1].

Let us recall succinctly the machinery of Barbasch-Moy's preservation of unitarity under the real central character assumption. The central characters of $\mathcal{H}(G, I$, triv $)$ are in one-to-one correspondence with $G$-conjugacy classes of semisimple elements $s$ in $G$. This is the semisimple element $s$ appearing in the correspondence (1.1.1). We say that a central character $s$ is real if the elliptic part of $s$ in the polar decomposition is central in $G$. The element $s$ is sometimes referred to as the infinitesimal character of the representation.

Theorem ([BM1]). In the equivalence of categories $\pi \mapsto \pi^{I}$ between $\operatorname{Iw}\left(\mathcal{G}(\mathbb{F})^{s}\right)$ and $\mathcal{H}(G, I$, triv)-mod, hermitian and unitary modules with real infinitesimal character correspond, respectively. 
One of the basic ingredients for the proof is the signature character of a representation introduced in Vo1. If $\pi$ is a hermitian representation in $\operatorname{lw}\left(\mathcal{G}(\mathbb{F})^{s}\right)$, let $\Sigma(\pi)$ denote its signature character, and $\theta_{K}(\pi)$ its $K$-character (see BM1). Similarly, if $\bar{\pi}$ is a module in $\mathcal{H}(G, I$, triv)-mod, let $\Sigma(\bar{\pi})$ denote its signature character, and $\theta_{W}(\bar{\pi})$ its $W$-character. (Recall that $\mathcal{H}(G, I$, triv) has the subalgebra of functions supported on $K$, which is isomorphic with the Weyl group $W$ of $G$.) The proof that $\pi^{I}$ unitary implies $\pi$ unitary (the other implications are straightforward) in BM1] has the following steps:

(1) There exist tempered representations $\pi_{1}, \ldots, \pi_{\ell}$ in $\operatorname{Iw}\left(\mathcal{G}(\mathbb{F})^{s}\right)$ such that

$$
\Sigma(\pi)=\left(\sum_{i} a_{i} \theta_{K}\left(\pi_{i}\right), \sum_{i} b_{i} \theta_{K}\left(\pi_{i}\right)\right),
$$

for some integers $a_{i}, b_{i}$. (This is proved by induction on the length of the Langlands parameter, and uses the fact that $\operatorname{Iw}\left(\mathcal{G}(\mathbb{F})^{s}\right)$ is closed under taking subquotients.)

(2) A module $\pi^{\prime}$ in $\operatorname{Iw}\left(\mathcal{G}(\mathbb{F})^{s}\right)$ is tempered if and only if $\left(\pi^{\prime}\right)^{I}$ is tempered in $\mathcal{H}(G, I$, triv)-mod. (One defines temperedness via Casselman's criterion, so the notion makes sense in both settings.)

$$
\Sigma_{W}\left(\pi^{I}\right)=\left(\sum_{i} a_{i} \theta_{W}\left(\pi_{i}^{I}\right), \sum_{i} b_{i} \theta_{W}\left(\pi_{i}^{I}\right)\right),
$$

for the same $a_{i}, b_{i}$ as in (1.2.1). (One needs here not only that the irreducibles correspond in the equivalence of categories, but also the fact that the standard modules correspond.)

(4) The set $\left\{\theta_{W}(\bar{\pi}): \bar{\pi}\right.$ is tempered with real central character $\}$ is linearly independent in the Grothendieck group of $W$, in fact, it gives a basis. (This proof is based on the geometric realization of the $W$-structure of tempered modules and the results of Borho-MacPherson.)

(5) Then one sees that if $\pi^{I}$ is unitary, $\Sigma\left(\pi^{I}\right)$ being positive definite, it implies by (4) that $b_{i}=0$, for all $i$, and so $\Sigma(\pi)$ is positive definite as well.

The essential step in this correspondence is (4). As stated, it does not hold true if one drops the "real central character" assumption. Even if one considers the weaker (but sufficient) case of tempered modules whose central character has a fixed elliptic part $s_{e}$, this statement is still false in general. The subject of BM2] is to show that, however, one still has that, for the tempered modules (with a fixed elliptic part $s_{e}$ ) which appear in the equation (1.2.2), $\sum_{i} b_{i} \theta_{W}\left(\pi_{i}^{I}\right)=0$ implies $b_{i}=0$. For this one uses (a variant of) the reduction to the graded Hecke algebra ( $\mathrm{Lu} 1]$.

In this setting, the spherical (equivalently, the generic Iwahori-spherical) unitary dual for all split $p$-adic groups is determined: for classical types in BM3 and Ba, for types $G_{2}, F_{4}$ in [Ci1, and for types $E$ in [BC1. By different methods, the complete unitary dual for types $G L(n)$ and $G_{2}$ had been previously obtained in Ta and $\mathrm{Mu}$, respectively.

For nongeneric modules (of types other than $A$ or $G_{2}$ ) less is known: the unitary dual with real central character for the graded Hecke algebra with equal parameters is known for $F_{4}$ ([Ci1] ) and $E_{6}$ ([Ci2 $)$. It is not known for classical groups, except in some small rank cases. 
When one attempts to extend theorem 1.2 to the categories Uni $(\mathcal{G}(\mathbb{F}), J, \rho)$, the first thing to prove is item (4) above: the linear independence of $W$-characters for tempered modules with real central character in the affine Hecke algebra with unequal parameters appearing on the geometric side. This is discussed in section 3 of this paper. We work, as we may since the central character is assumed real, in the equivalent setting of the graded Hecke algebra.

1.3. In BC1, one proposed a direct method of matching signatures of hermitian forms for irreducible modules of a graded Hecke algebra with equal parameters $\mathbb{H}$ with signatures of hermitian forms on spherical modules of smaller graded Hecke algebras $\mathbb{H}(\mathcal{O})$ constructed from root systems of centralizers of nilpotent complex orbits $\mathcal{O}$. This method proved particularly effective in the determination of the unitary generic modules, but it can be applied for the signatures of nongeneric modules as well.

In this paper, we apply this idea to the setting of geometric Hecke algebras $\mathbb{H}$ with unequal parameters ( $\mathrm{Lu} 2, \mathrm{Lu} 3, \mathrm{Lu} 4])$. As an application, we determine the unitary dual of certain Hecke algebras of types $G_{2}$ and $F_{4}$, with unequal parameters, which appear in Lu6], in the classification of unipotent representations of quasi-simple $p$-adic groups.

We present next the philosophy of matching signatures and unitary duals. The geometric classification of $\mathbb{H}$ is controlled by a complex group $G$, with Lie algebra $\mathfrak{g}$, and Cartan subalgebra $\mathfrak{t}$, a Levi subgroup $L$ with Lie algebra $\mathfrak{l}$, and a cuspidal local system $\mathcal{L}$ supported on some nilpotent orbit $\mathcal{C}$ of $\mathfrak{l}$ (see section 3 ). Note that the rank of $G$ may be larger than that of $\mathbb{H}$, e.g., the most interesting example in this paper is an algebra of type $F_{4}$ parameterized by $G$ simply connected of type $E_{7}$. Let $V$ be a simple $\mathbb{H}$-module. The graded Hecke algebra version of the geometric classification (1.1.1) says that $V$ is parameterized by a triple $\left(s, \mathcal{O}_{e}, \psi\right)$, where $s$ is a (conjugacy class of a) semisimple element in $\mathfrak{t}, \mathcal{O}_{e}$ is a $Z_{G}(s)$-orbit on $\mathfrak{g}_{1}=\{x \in \mathfrak{g}:[s, x]=x\}$, and $\psi$ is a certain representation of the component group $A(s, e)$. The orbit $\mathcal{C}$ is necessarily distinguished in $\mathfrak{l}$ (in the sense of Bala-Carter), and there are restrictions about which $\psi$ can appear depending on the cuspidal local system $\mathcal{L}$. The nilpotent representative $e$ can be completed to a Jacobson-Morozov triple $\{e, h, f\}$ of $\mathcal{O}=G \cdot \mathcal{O}_{e}$, such that $h \in \mathfrak{t}$. Let $Z(\mathcal{O})$ denote the centralizer in $G$ of $\{e, h, f\}$. It is a reductive group, possibly disconnected. Let $A(\mathcal{O})$ denote its group of components, and $\mathfrak{z}(\mathcal{O})$ its Lie algebra. Then $s$ can be written as

$$
s=\frac{1}{2} h+\nu
$$

where $\nu$ is a semisimple element of $\mathfrak{z}(\mathcal{O})$.

Now fix a nilpotent orbit $\mathcal{O}$ in $\mathfrak{g}$, together with a representation $\phi$ of the component group $A(\mathcal{O})$. The requirement is that the pair $(\mathcal{O}, \phi)$ appears in the generalized Springer correspondence ([L5]), and so it has a representation $\mu_{0}$ of the Weyl group $W$ defining $\mathbb{H}$ attached to it. This is not the Weyl group of $G$, but rather $W=N_{G}(L) / L$. (The fact that this is a Coxeter group is due to the very particular form $L$ must have in order to allow a cuspidal local system.) Let us denote by $\mathcal{U}(\mathcal{O}, \phi)$ the set of unitary $\mathbb{H}$-modules with lowest $W$-type $\mu_{0}$, in the sense of sections 3.4 3.6. The triple $\left(s, \mathcal{O}_{e}, \psi\right)$ parameterizing a module $V$ with lowest $W$-type $\mu_{0}$ must satisfy $\mathcal{O}_{e} \subset \mathcal{O}$, and $\operatorname{Hom}_{A(s, e)}[\phi: \psi] \neq 0$. 
We attach a Hecke algebra $\mathbb{H}(\mathfrak{z}(\mathcal{O}), c(\phi))$ with the root system that of $\mathfrak{z}(\mathcal{O})$, and certain parameters $c(\phi)$ depending on $\phi$, and consider $\mathcal{S U}(\mathbb{H}(\mathfrak{z}(\mathcal{O}), c(\phi)))$, the set of unitary spherical $\mathbb{H}(\mathfrak{z}(\mathcal{O}), c(\phi))$-modules. By the equation (1.3.1), $s \mapsto \nu$, to a module $V=V_{s, \mathcal{O}_{e}, \psi}$ with lowest $W$-type $\mu_{0}$, we may attach a spherical module $\rho(V)$ of $\mathbb{H}(\mathfrak{z}(\mathcal{O}), c(\phi))$ parameterized by $\nu$ (i.e., the spherical subquotient of the minimal principal series defined by $\nu$ ).

The hope would be that, in this way, the set $\mathcal{U}(\mathcal{O}, \phi)$ can be put in correspondence with $\mathcal{S U}(\mathbb{H}(\mathfrak{z}(\mathcal{O}), c(\phi)))$. This is motivated by the case of generic unitary modules of Hecke algebras with equal parameters (Ba, BC1, Ci1]), where a particular case of this correspondence worked almost perfectly. More precisely, that was the case of $\phi=t r i v$, so that $\mathbb{H}$ is a Hecke algebra with equal parameters of the same type as $G$ ( $L$ is a maximal torus, and $\mathcal{C}=0$ ). The attached Hecke algebra is $\mathbb{H}(\mathfrak{z}(\mathcal{O}), 1)$, meaning that it has equal parameters too. Let us denote by $\mathcal{G U}(\mathcal{O}$, triv $)$ and $\mathcal{G S U}(\mathbb{H}(\mathfrak{z}(\mathcal{O}), 1)$ the corresponding subsets consisting of (unitary) generic representations, in the sense of containing the sign $W$-type. It was shown in the above references that, except for a handful of cases (none in the classical types, in $G_{2}$ or $E_{6}$, one in $F_{4}$ and in $E_{7}$, and six in $\left.E_{8}\right)$, the sets $\mathcal{G U}(\mathcal{O}$, triv $)$ and $\mathcal{G S U}(\mathbb{H}(\mathfrak{z}(\mathcal{O}), 1)$ are in one-to-one correspondence. In more generality, such a relation is not as perfect, but it still gives serious information about $\mathcal{U}(\mathcal{O}, \phi)$.

There are also two immediate technical problems. One obvious problem is that, in general, an irreducible module may have more than one lowest $W$-type, so it is actually better to match $\mathcal{U}(\mathcal{O}, \phi)$ with a "quasi-spherical" unitary dual of an extension (by outer automorphisms) of the Hecke algebra defined from $\mathfrak{z}(\mathcal{O})$. A second issue is that, unlike the case of representations of real reductive groups, a lowest $W$-type may appear with multiplicity greater than one. But if this is the case, it is a fact (only empirical to our knowledge) that there exists another lowest $W$-type with multiplicity one. This fact is needed in order to be able to define a normalization of the intertwining operators involved in the calculation of signatures of hermitian forms. However, this second difficulty only arises for geometric algebras with equal parameters. We ignore these problems for now and refer to BC1 and Ci2 for details and examples of these phenomena.

In order to exhibit a relation between $\mathcal{U}(\mathcal{O}, \phi)$ and $\mathcal{S U}(\mathbb{H}(\mathfrak{z}(\mathcal{O}), \phi))$, for every $(\mathcal{O}, \phi)$, we find a set of $W$-types $\left\{\mu_{0}, \mu_{1}, \ldots, \mu_{l}\right\}$, and a corresponding set of $W(\mathfrak{z}(\mathcal{O}))$-representations $\left\{\rho\left(\mu_{0}\right), \rho\left(\mu_{1}\right), \ldots, \rho\left(\mu_{l}\right)\right\}$,

$$
\mu_{j} \longleftrightarrow \rho\left(\mu_{j}\right),
$$

such that:

(1) if $V$ is hermitian but $\rho(V)$ is not, then the hermitian form on $V$ is indefinite, i.e., $V$ is not unitary;

(2) if $V$ is hermitian and $\rho(V)$ is hermitian, then the signature of the hermitian form of $V$ restricted to the $W$-type $\mu_{j}$ is identical with the signature of the hermitian form of $\rho(V)$ restricted to the $W(\mathfrak{z}(\mathcal{O}))$-representation $\rho\left(\mu_{j}\right)$, for all $0 \leq j \leq l$.

We explain the relation between $\mu_{j}$ and $\rho\left(\mu_{j}\right)$ (see [BC1]). Let $\mathfrak{m} \supset \mathfrak{l}$ denote a Levi subalgebra of $\mathfrak{g}$, which is Bala-Carter for $\{e, h, f\}$, and let $M \subset G$ be the corresponding Levi subgroup. So $e$ is distinguished in $\mathfrak{m}$. There exists a geometric Hecke subalgebra $\mathbb{H}_{M}$ of $\mathbb{H}$ which is parameterized by $(L, \mathcal{L})$ in $M$. By Lu4, the irreducible $\mathbb{H}_{M}$-modules parameterized by $\left(h, e, \psi^{\prime}\right)$, for any allowable $\psi^{\prime} \in$ 
$\widehat{A_{M}(s, e)}$, are discrete series. We find a $\psi^{\prime}$ such that via the natural map $A_{M}(h, e)=$ $A_{M}(s, e) \rightarrow A(s, e), \operatorname{Hom}_{A_{M}}(h, e)\left[\psi, \psi^{\prime}\right] \neq 0$, and realize $V_{s, e, \psi}$ as a summand of the Langlands subquotient of the standard induced module $X(M, \sigma, \nu)=\mathbb{H} \otimes_{\mathbb{H}_{M}}(\sigma \otimes$ $\left.\mathbb{C}_{\nu}\right)$. Then $\operatorname{Hom}_{W}\left[\mu_{j}: X(M, \sigma, \nu)\right]$ carries a natural action of $W(\mathfrak{z}(\mathcal{O}))$ (independent of $\nu$ ) and we call the resulting representation $\rho\left(\mu_{j}\right)$.

Of course, this procedure is efficient only if we find a sufficiently large number of matching types in this way. This is our main criterion for proving nonunitarity of $\mathbb{H}$-modules. Ideally, if sufficiently many $W$-types are matched, one would be able to conclude that a hermitian module $V$ is unitary only if $\rho(V)$ is unitary. This doesn't always happen, and in fact we find examples here of $\mathcal{U}(\mathcal{O}, \phi)$ 's both smaller or larger than expected. We exemplify this at the end of the introduction.

There is also the case of spherical unitary $\mathbb{H}$-modules, whose lowest $W$-type is trivial. In that case, $\mathcal{O}=G \cdot \mathcal{C}$ (it is the minimal orbit appearing in the parameterization), $\mathfrak{z}(\mathcal{O})$ has the same type as $\mathbb{H}$, and the above correspondence is a tautology. We determine the spherical unitary $\mathbb{H}$-modules by other methods, known to the experts. In particular, we use the Iwahori-Matsumoto involution $I M$ (definition (4.2.1), which preserves unitarity. If $V$ is a spherical $\mathbb{H}$-module, then $I M(V)$ contains the sign $W$-type. If $I M(V) \neq V$, then $I M(V)$ is parameterized by a larger orbit, for which the unitary set has already been determined. But in order to apply this method, we need to determine explicitly which unitary irreducible modules have the sign $W$-type for any given pair $\left(\mathcal{O}^{\prime}, \phi^{\prime}\right)$. This is achieved by computing composition series as part of section [5.3 and the result is in Table 4

So this approach leaves the case $V=I M(V)$ and $V$ spherical, equivalently, $V$ must be an irreducible spherical principal series. The unitarity of such representations is discussed in section 5.5, and the methods are again well-known: continuous deformations of parameters and unitary induction, but also certain explicit calculation of signatures.

1.4. We give an outline of the paper. In sections 2.1 2.7, we present the necessary definitions and background on the representation theory of affine and graded Hecke algebras, assuming the parameters are arbitrary. We will mostly work with the affine graded Hecke algebra. In particular, we recall the Langlands classification (actually, the reduction to tempered modules) as in Ev, the unitarity of tempered modules, as it follows from Op1, and the results about the Hermitian forms and intertwining operators from BM3. In section 2.8, we present a type of reduction to real central ("infinitesimal") character for the unitary dual of the Hecke algebra, which is the complete analogue of the result for real reductive groups, as in $[\mathrm{Kn}$, XVI.4. This is well known to the specialists.

From section 3 on, we restrict to the case of geometric Hecke algebras. We recall the relevant results about the classification of simple $\mathbb{H}$-modules, and the generalized Springer correspondence ([L5]). An important consequence is that the set of irreducible tempered $\mathbb{H}$-modules with real central character are linearly independent in the Grothendieck group of $W$. Therefore, the method of BM1 holds, and the correspondence with unitary (unipotent) representations of $p$-adic groups can be established. So for geometric Hecke algebras, the unitarity of tempered $\mathbb{H}$-modules could also be obtained from the correspondence with the $p$-adic group.

In section 4, we analyze Hermitian forms and intertwining operators for simple modules. We restrict, as we may, to modules with real central character. As an easy application, we present the spherical unitary dual for type $G_{2}$ with arbitrary 
unequal parameters. (In fact, if we factor in the Iwahori-Matsumoto involution, and the tempered modules, one obtains all the unitary modules with real central character of $G_{2}$.)

Section 5 presents a more interesting application. We determine the unitary dual of the Hecke algebra (of type $F_{4}$ ) constructed in Lu2 from a cuspidal local system on the principal nilpotent orbit in the Levi $\left(3 A_{1}\right)^{\prime \prime}$ of the simply connected $E_{7}$. Besides the equal parameter case, this is, essentially, the only Hecke algebra of type $F_{4}$ which appears in the classification of unipotent representations of $p$-adic groups from [Lu6] (the equal parameter $\mathbb{H}\left(F_{4}\right)$ was treated in Ci1]). We also remark that the Hecke algebras of types $B / C$ with unequal parameters which appear in the classification of the unipotent exceptional $p$-adic groups are parabolic subalgebras of this one.

The main results are theorem 5.1] proposition and corollary [5.5. Although the methods employed are mostly uniform, details need to be checked case by case for each nilpotent orbit in $E_{7}$ which appears in the classification (see Figure 21). As mentioned above, we use the fact that $I M$ preserves unitarity of modules. In order to use $I M$, we need to compute the decompositions of standard modules and the $W$-structure of unitary modules.

As a consequence of the calculations with intertwining operators in section 5 , we obtain the $W$-structure of standard modules for the cuspidal local system $\left(3 A_{1}\right)^{\prime \prime}$ in $E_{7}$ (section 5.6). This is also (at least in principle) computable by the generalized Green polynomials algorithms in Lu7]. An implementation of such algorithms for small rank classical groups was realized in [LS].

1.5. Examples. We conclude the introduction with two examples relative to the matching of signatures and unitary duals for this unequal parameter $\mathbb{H}$ of type $F_{4}$. The nilpotent orbits are of type $E_{7}$.

(a) $\mathcal{O}=A_{2}+3 A_{1}, A(\mathcal{O})=\mathbb{Z} / 2 \mathbb{Z}, \mathfrak{z}(\mathcal{O})=G_{2}, \phi=$ sgn. The intertwining operator calculations indicate a matching with the spherical unitary dual of a Hecke algebra $\mathbb{H}\left(G_{2},(2,1)\right)$ : type $G_{2}$ with parameter 2 on the long root and 1 on the short root. $W\left(G_{2}\right)$ has six representations, in the notation of $\mathrm{Ca}$ : $(1,0),(1,3)^{\prime},(1,3)^{\prime \prime},(1,6),(2,1),(2,2)$. There are two minimal subsets of $\widehat{W\left(G_{2}\right)}$ which determine $\mathcal{S U}\left(\mathbb{H}\left(G_{2},(2,1)\right)\right.$, in the sense that a hermitian module is unitary if and only if the hermitian form is positive definite on this set of types: $\{(1,0),(2,1),(2,2)\}$ or $\left\{(1,0),(1,3)^{\prime},(1,3)^{\prime \prime},(2,1)\right\}$. It turns out there are $W\left(F_{4}\right)$ types $\mu_{j}$ such that one realizes as $\rho\left(\mu_{j}\right)$ (see the explanation around equation $(1.3 .2)$ ) the $W\left(G_{2}\right)$-representations $\left\{(1,0),(1,3)^{\prime},(2,1),(1,6)\right\}$. So we cannot conclude that $\mathcal{U}\left(A_{2}+3 A_{1}, \operatorname{sgn}\right)$ is a subset of $\mathcal{S U}\left(\mathbb{H}\left(G_{2},(2,1)\right)\right.$, and in fact, we find that $\mathcal{U}\left(A_{2}+3 A_{1}, \operatorname{sgn}\right) \supsetneq \mathcal{S U}\left(\mathbb{H}\left(G_{2},(2,1)\right)\right.$ (see Table 3 and Figure 3).

(b) $\mathcal{O}=D_{4}\left(a_{1}\right)+A_{1}, A(\mathcal{O})=(\mathbb{Z} / 2 \mathbb{Z})^{2}, \mathfrak{z}(\mathcal{O})=2 A_{1}$. There are two representations $\phi_{1}$ and $\phi_{2}$ of $A(\mathcal{O})$ which enter the generalized Springer correspondence with $\widehat{W\left(F_{4}\right)}([\mathrm{Spa}]): \phi_{1}$ corresponds to a nine-dimensional $W\left(F_{4}\right)$-representation, and $\phi_{2}$ to a two-dimensional one. We consider here $\phi_{1}$. The calculations indicate that the matchings should be with the spherical unitary dual of $\mathbb{H}\left(2 A_{1},(5,5)\right)$ : type $A_{1}+A_{1}$, and the parameter is 5 on both simple roots. The set $\mathcal{S U}\left(\mathbb{H}\left(2 A_{1},(5,5)\right)\right.$ is detected on $\{t r i v \otimes t r i v, t r i v \otimes s g n, s g n \otimes t r i v\} \subset \widehat{W\left(2 A_{1}\right)}$. All three representations are matched by certain $\mu_{j}$ 's, so we can conclude that $\mathcal{U}\left(D_{4}\left(a_{1}\right)+A_{1}, \phi_{1}\right) \subset$ 
$\mathcal{S U}\left(\mathbb{H}\left(2 A_{1},(5,5)\right)\right.$. The problem here is that there is extra reducibility in the standard modules for $\mathbb{H}$ parameterized by $(\mathcal{O}, \phi)$ which is not detected by the hermitian form on the $W\left(F_{4}\right)$-types $\mu_{j}$. What we find is that $\mathcal{U}\left(D_{4}\left(a_{1}\right)+A_{1}, \phi_{1}\right) \subsetneq$ $\mathcal{S U}\left(\mathbb{H}\left(2 A_{1},(5,5)\right)\right.$ (see Table 3$)$.

\section{Preliminaries}

2.1. Let $(X, \check{X}, R, \check{R}, \Pi)$ be a fixed based root datum. Denote by $R^{+}$the positive roots determined by $\Pi$, and by $\check{R}^{+}$the corresponding positive coroots. Define $\mathfrak{h}=\check{X} \otimes_{\mathbb{Z}} \mathbb{C}$ and $\mathfrak{h}^{*}=X \otimes_{\mathbb{Z}} \mathbb{C}$. Let $W$ be the finite Weyl group generated by the set $S$ of simple reflections in the roots of $\Pi$. Denote by $Q=\mathbb{Z} R \subset X$ the root lattice, and set $\Omega=X / Q$.

Let $\widetilde{W}=W \ltimes X$ be the extended Weyl group, and $W_{\text {aff }}=W \ltimes Q$ be the affine Weyl group with the set of simple affine reflections $S_{\text {aff. }}$. Recall that $W_{\text {aff }}$ is normal in $\widetilde{W}$, and $\widetilde{W} / W_{\text {aff }} \cong \Omega$. Let $\ell: \widetilde{W} \rightarrow \mathbb{Z}_{>0}$ denote the length function ([u1): it is the extension of the (Coxeter) length function of $W_{\text {aff }}$ and it is identically 0 on $\Omega$.

2.2. Let $c: R \rightarrow \mathbb{Z}_{>0}$ be a function such that $c_{\alpha}=c_{\beta}$, whenever $\alpha$ and $\beta$ are $W$-conjugate. Let $r$ denote an indeterminate. As a vector space,

$$
\mathbb{H}=\mathbb{C}[W] \otimes \mathbb{C}[r] \otimes \mathbb{A},
$$

where $\mathbb{A}$ is the symmetric algebra over $\mathfrak{h}^{*}$. The generators are $t_{w} \in \mathbb{C}[W], w \in W$ and $\omega \in \mathfrak{h}^{*}$. The relations between the generators are:

$$
\begin{array}{lr}
t_{w} t_{w}^{\prime}=t_{w w^{\prime}}, & \text { for all } w, w^{\prime} \in W ; \\
t_{s}^{2}=1, & \text { for any simple reflection } s \in W ; \\
\omega t_{s}=t_{s} s(\omega)+r c_{\alpha} \omega(\check{\alpha}), & \text { for simple reflections } s=s_{\alpha} \in S, w \in g^{*} .
\end{array}
$$

Assume the root system $R$ is irreducible. If $R$ is simply-laced, the function $c$ must be constant. In the two root lengths case, we will denote the function $c$ by the pair $\left(c_{l}, c_{s}\right) \in \mathbb{Z}_{>0}^{2}$, where $c_{l}, c_{s}$ specify the values of $c$ on the long and short roots, respectively (in this order). Let $k \in \mathbb{Z}_{>0}$ be the ratio $k=\left(\alpha_{l}, \alpha_{l}\right) /\left(\alpha_{s}, \alpha_{s}\right)$ for some long root $\alpha_{l}$ and short root $\alpha_{s}$. Then it is straightforward to see that $\mathbb{H}\left(R,\left(c_{1}, c_{2}\right)\right) \cong \mathbb{H}\left(\check{R},\left(k c_{2}, c_{1}\right)\right)$. For example, $\mathbb{H}\left(F_{4},(1,2)\right) \cong \mathbb{H}\left(F_{4},(4,1)\right)$.

2.3. By [Lu1, the center of $\mathbb{H}$ is $\mathbb{C}[r] \otimes \mathbb{A}^{W}$. On any simple (finite dimensional) $\mathbb{H}-$ module, the center of $\mathbb{H}$ acts by a character, which we will call a central character. The central characters correspond to $W$-conjugacy classes of semisimple elements $\left(r_{0}, s\right) \in \mathbb{C} \oplus \mathfrak{h}$.

We decompose $\mathfrak{h}$ into real and imaginary parts; the complex conjugation ${ }^{-}$of $\mathbb{C}$ induces a conjugation on $\mathfrak{h}$. We set $\mathfrak{h}_{\mathbb{R}}=\{a \in \mathfrak{h}: \bar{a}=a\}$ and $\mathfrak{h}_{i \mathbb{R}}=\{a \in \mathfrak{h}: \bar{a}=$ $-a\}$. For every $s \in \mathfrak{h}$, we then have a unique decomposition $s=\operatorname{Re}(s)+\operatorname{Im}(s)$, where $\operatorname{Re}(s) \in \mathfrak{h}_{\mathbb{R}}$ and $\operatorname{Im}(s) \in \mathfrak{h}_{i \mathbb{R}}$.

Definition. A central character $\left(r_{0}, s\right)$ is called real if $\left(r_{0}, s\right) \in \mathbb{R} \oplus \mathfrak{h}_{\mathbb{R}}$.

2.4. $\mathbb{H}$ has a $*$-operation given on generators as follows (as in BM2], section 5):

$$
\begin{aligned}
& t_{w}^{*}=t_{w^{-1}}, w \in W ; r^{*}=r ; \\
& \omega^{*}=-\bar{\omega}+r \sum_{\alpha \in R^{+}} c_{\alpha} \bar{\omega}(\check{\alpha}) t_{s_{\alpha}}, \omega \in \mathfrak{h}^{*} .
\end{aligned}
$$


The $\mathbb{H}$-module $V$ is called Hermitian if it admits a Hermitian form $\langle$,$\rangle such$ that

$$
\left\langle x \cdot v_{1}, v_{2}\right\rangle=\left\langle v_{1}, x^{*} \cdot v_{2}\right\rangle \text {, for all } v_{1}, v_{2} \in V, x \in \mathbb{H} .
$$

It is called unitary, if in addition the Hermitian form is positive definite.

2.5. We present the Langlands classification for $\mathbb{H}$ as in $\mathrm{Ev}$.

If $V$ is a (finite dimensional) simple $\mathbb{H}$-module, $\mathbb{A}$ induces a generalized weight space decomposition

$$
V=\bigoplus_{\lambda \in \mathfrak{h}} V_{\lambda}
$$

Call $\lambda$ a weight if $V_{\lambda} \neq 0$.

Definition. The irreducible module $\sigma$ is called tempered if $\omega_{i}(\lambda) \leq 0$, for all weights $\lambda \in \mathfrak{h}$ of $\sigma$ and all fundamental weights $\omega_{i} \in \mathfrak{h}^{*}$. If $\sigma$ is tempered and $\omega_{i}(\lambda)<0$, for all $\lambda, \omega_{i}$ as above, $\sigma$ is called a discrete series.

For every $\Pi_{M} \subset \Pi$, define $R_{M} \subset R$ to be the set of roots generated by $\Pi_{M}$, $\check{R}_{M} \subset \check{R}$ the corresponding set of coroots, and $W(M) \subset W$ the corresponding Weyl subgroup. Let $\mathbb{H}_{M}$ be the Hecke algebra attached to $\left(\mathfrak{h}, R_{M}\right)$. It can be regarded naturally as a subalgebra of $\mathbb{H}$.

Define $\mathfrak{t}=\left\{\nu \in \mathfrak{h}:\langle\alpha, \nu\rangle=0\right.$, for all $\left.\alpha \in \Pi_{M}\right\}$ and $\mathfrak{t}^{*}=\left\{\lambda \in \mathfrak{h}^{*}:\langle\lambda, \check{\alpha}\rangle=\right.$ 0 , for all $\left.\alpha \in \Pi_{M}\right\}$. Then $\mathbb{H}_{M}$ decomposes as

$$
\mathbb{H}_{M}=\mathbb{H}_{M_{0}} \otimes S\left(\mathfrak{t}^{*}\right),
$$

where $\mathbb{H}_{M_{0}}$ is the Hecke algebra attached to $\left(\mathbb{C}\left\langle\Pi_{M}\right\rangle, R_{M}\right)$.

We will denote by $I(M, U)$ the induced module $I(M, U)=\mathbb{H} \otimes_{\mathbb{H}_{M}} U$.

Theorem $([\mathrm{Ev}])$.

(1) Every irreducible $\mathbb{H}$-module is a quotient of a standard induced module $X(M, \sigma, \nu)=I\left(M, \sigma \otimes \mathbb{C}_{\nu}\right)$, where $\sigma$ is a tempered module for $\mathbb{H}_{M_{0}}$, and $\nu \in \mathfrak{t}^{+}=\left\{\nu \in \mathfrak{t}: \operatorname{Re}(\alpha(\nu))>0\right.$, for all $\left.\alpha \in \Pi \backslash \Pi_{M}\right\}$.

(2) Assume the notation from (1). Then $X(M, \sigma, \nu)$ has a unique irreducible quotient, denoted by $L(M, \sigma, \nu)$.

(3) If $L(M, \sigma, \nu) \cong L\left(M^{\prime}, \sigma^{\prime}, \nu^{\prime}\right)$, then $M=M^{\prime}, \sigma \cong \sigma^{\prime}$ as $\mathbb{H}_{M_{0}}$-modules, and $\nu=\nu^{\prime}$.

We will call a triple $(M, \sigma, \nu)$ as in theorem 2.5 a Langlands parameter.

A similar classification holds with $\nu \in \mathfrak{t}_{*}^{-}$(antidominant). In that case, $X(M, \sigma, \nu)$ has a unique irreducible submodule $L(M, \sigma, \nu)$

2.6. We need to recall some results about the affine (Iwahori-)Hecke algebra as in Op1, and their implications for $\mathbb{H}$. Fix $q>1$. The affine Hecke algebra $\mathcal{H}$ is the associative $\mathbb{C}$-algebra with basis $\left\{T_{w}: w \in \widetilde{W}\right\}$ and set of parameters $d: \Pi_{\text {aff }} \rightarrow \mathbb{Z}_{>0}$ which satisfies the relations:

(1) $T_{w} T_{w^{\prime}}=T_{w w^{\prime}}$ if $w, w^{\prime} \in \widetilde{W}$, with $\ell\left(w w^{\prime}\right)=\ell(w)+\ell\left(w^{\prime}\right)$;

(2) $\left(T_{s_{\alpha}}+1\right)\left(T_{s_{\alpha}}-q^{d_{\alpha}}\right)=0$, for all simple affine reflections $s_{\alpha} \in S_{\text {aff }}$.

This is the Iwahori-Matsumoto presentation of $\mathcal{H}$. 
The $*$-operation for $\mathcal{H}$ is given by $T_{w}^{*}=T_{w^{-1}}$. The algebra $\mathcal{H}$ has a trace, i.e., a linear functional $\tau: \mathcal{H} \rightarrow \mathbb{C}$, given by

$$
\tau\left(T_{w}\right)=\left\{\begin{array}{ll}
1, & w=1, \\
0, & w \neq 1,
\end{array} \quad w \in \widetilde{W} .\right.
$$

One checks that $\tau$ defines an inner product on $\mathcal{H}$ via

$$
(x, y):=\tau\left(x^{*} y\right), x, y \in \mathcal{H},
$$

and the basis $\left\{T_{w}: w \in \widetilde{W}\right\}$ is orthogonal with respect to $($,$) .$

Definition. Let $\mathfrak{H}$ be the Hilbert space completion of $\mathcal{H}$ with respect to ( , ).

2.7. The algebra $\mathcal{H}$ admits a second presentation due to Bernstein and Lusztig. The generators are $\left\{T_{w}: w \in W\right\}$ and $\left\{\theta_{x}: x \in X\right\}$ with relations:

(1) $T_{w} T_{w^{\prime}}=T_{w w^{\prime}}$ if $w, w^{\prime} \in W$, with $\ell\left(w w^{\prime}\right)=\ell(w)+\ell\left(w^{\prime}\right)$;

(2) $\left(T_{s_{\alpha}}+1\right)\left(T_{s_{\alpha}}-q^{d_{\alpha}}\right)=0$, for all simple reflections $s_{\alpha} \in S$;

(3) $\theta_{x} \theta_{x}^{\prime}=\theta_{x+x^{\prime}}, x, x^{\prime} \in X$, and $\theta_{0}=1$;

(4) $\theta_{x} T_{s_{\alpha}}-T_{s_{\alpha}} \theta_{s_{\alpha}(x)}=\left(q^{d_{\alpha}}-1\right) \frac{\theta_{x}-\theta_{s_{\alpha}(x)}}{1-\theta_{-\alpha}}, x \in X, s_{\alpha} \in S$.

Denote by $\mathcal{H}_{W}$ and $\mathcal{A}$ the subalgebras generated by $\left\{T_{w}\right\}$ and $\left\{\theta_{x}\right\}$, respectively. We refer the reader to Lu1 for the more general version of this definition of $\mathcal{H}$ and for the relation between the two presentations.

Note that the trace $\tau$ is easy to define using the Iwahori-Matsumoto presentation, but not so if one uses instead the Bernstein-Lusztig presentation. (See Op2 for details.) Similarly to section 2.5. one defines tempered and discrete series modules for $\mathcal{H}$ using the weights of the abelian subalgebra $\mathcal{A}$ ("Casselman's criteria", see Lu1 and Op1).

Proposition (Op1, 2.22). A finite dimensional irreducible representation $\sigma$ of $\mathcal{H}$ is a discrete series if and only if it is a subrepresentation of $\mathfrak{H}$. In particular, $\sigma$ is a unitary module of $\mathcal{H}$.

A standard argument then implies the unitarity of irreducible tempered $\mathcal{H}$ modules as well. Moreover, we can transfer this result to the graded Hecke algebra, using the correspondence between tempered modules of $\mathcal{H}$ and tempered modules of $\mathbb{H}$ (theorem 9.3 in [Lu1]) and the preservation of unitarity (theorem 4.3 in [BM2]).

2.8. Given a module $V$, let $V^{h}$ denote the Hermitian dual. Every element $x \in \mathbb{H}$ can be written uniquely as $x=\sum_{w \in W / W(M)} t_{w} x_{w}$, with $x_{w} \in \mathbb{H}_{M}$. Let $\epsilon_{M}: \mathbb{H} \rightarrow \mathbb{H}_{M}$ be the map defined by $\epsilon_{M}(x)=x_{1}$, that is, the component of the identity element $1 \in W$. In the particular case $\Pi_{M}=\emptyset$, we will denote the map by $\epsilon: \mathbb{H} \rightarrow \mathbb{A}$.

Lemma ([BM3, 1.4). If $U$ is a module for $\mathbb{H}_{M}$, and $\langle,\rangle_{M}$ denotes the Hermitian pairing with $U^{h}$, then the Hermitian dual of $I(M, U)$ is $I\left(M, U^{h}\right)$, and the Hermitian pairing is given by

$$
\left\langle t_{x} \otimes v_{x}, t_{y} \otimes v_{y}\right\rangle_{h}=\left\langle\epsilon_{M}\left(t_{y}^{*} t_{x}\right) v_{x}, v_{y}\right\rangle_{M}, x, y \in W / W(M), v_{x}, v_{y} \in U .
$$

Applying this result to a Langlands parameter $(M, \sigma, \nu)$ as in section 2.5 we find that the Hermitian dual of $X(M, \sigma, \nu)$ is $I\left(M, \sigma \otimes \mathbb{C}_{-\bar{\nu}}\right)$.

Let $w_{0}$ denote the longest Weyl group element in $W$, and let $W\left(w_{0} M\right)$ be the subgroup of $W$ generated by the reflections in $w_{0} R_{M}$. Let $w_{m}$ denote a shortest element in the double coset $W\left(w_{0} M\right) w_{0} W(M)$. Then $w_{m} \Pi_{M}$ is a subset of $\Pi$, which we denote by $\Pi_{w_{m} M}$. 
Proposition ([BM3, 1.5). The Hermitian dual of the irreducible Langlands quotient $L(M, \sigma, \nu)$ is $L\left(w_{m} M, w_{m} \sigma,-w_{m} \bar{\nu}\right)$. In particular, $L(M, \sigma, \nu)$ is Hermitian if and only if there exists $w \in W$ such that

$$
w M=M, \quad w \sigma \cong \sigma \quad \text { and } \quad w \nu=-\bar{\nu} .
$$

If this is the case, we will denote by $a_{w}: w \sigma \rightarrow \sigma$ the corresponding isomorphism. Let $w=s_{1} \ldots s_{k}$ be a reduced decomposition of $w$. For each simple root $\alpha$, define

$$
r_{s_{\alpha}}=t_{s_{\alpha}} \alpha-c_{\alpha} ; \quad r_{w}=r_{s_{\alpha_{1}}} \ldots r_{s_{\alpha_{k}}} .
$$

Lemma 1.6 in BM3 (based on proposition 5.2 in Lu1) proves that $r_{w}$ does not depend on the reduced expression of $w$.

Assume $L(M, \sigma, \nu)$ is Hermitian. Define

$$
\mathcal{A}(M, \sigma, \nu): X(M, \sigma, \nu) \rightarrow I\left(M, \sigma \otimes \mathbb{C}_{-\bar{\nu}}\right), x \otimes\left(v \otimes 1_{\nu}\right) \mapsto x r_{w} \otimes\left(a_{w}(v) \otimes 1_{-\bar{\nu}}\right) .
$$

One can verify, as in section 1.6 of [BM3, that this is an intertwining operator. The image of $\mathcal{A}(M, \sigma, \nu)$ is the Langlands quotient $L(M, \sigma, \nu)$.

2.9. Let $(M, \sigma, \nu)$ be a Langlands parameter as in section 2.5. and $\nu=\operatorname{Re} \nu+\operatorname{Im} \nu$ with $\operatorname{Im} \nu \neq 0$. Set

$$
R_{M_{1}}=\{\alpha \in R:\langle\operatorname{Im} \nu, \alpha\rangle=0\}, \quad R_{N_{1}}=\{\alpha \in R:\langle-\sqrt{-1} \operatorname{Im} \nu, \alpha\rangle>0\} .
$$

Clearly, $\Pi_{M} \subset R_{M_{1}}$. Moreover, $R_{M_{1}}$ is a root subsystem of $R$. Set $R_{M_{1}}^{+}=R_{M_{1}} \cap R^{+}$, and let $\Delta_{M_{1}}$ denote the set of corresponding simple roots. Note that $\Pi_{M} \subset \Delta_{M_{1}}$, but $\Delta_{M_{1}}$ need not be a subset of $\Pi$. But $\mathbb{H}_{M}$ is naturally a subalgebra of $\mathbb{H}_{M_{1}}$.

Assume $L(M, \sigma, \nu)$ is Hermitian and let $w \in W$ be as in proposition 2.8, From $w \nu=-\bar{\nu}$, it follows that $w \in W\left(M_{1}\right)$.

The triple $(M, \sigma, R e \nu)$ is a Langlands triple for $\mathbb{H}_{M_{1}}$, so it makes sense to consider $\sigma_{1}=L_{M_{1}}(M, \sigma$, Re $\nu)$. Moreover, $\sigma_{1}$ is Hermitian in $\mathbb{H}_{M_{1}}$.

Proposition. With the notation as above,

$$
I\left(M_{1},\left(\sigma_{1} \otimes \mathbb{C}_{I m \nu}\right)\right) \cong L(M, \sigma, \nu) .
$$

Proof. It is known that there exists $w^{\prime} \in W$ (of shortest length) which maps $\Delta_{M_{1}}$ onto a subset $\Pi_{M^{\prime}}$ of $\Pi$. This defines an isomorphism $a_{w^{\prime}}: \mathbb{H}_{M_{1}} \rightarrow \mathbb{H}_{M^{\prime}}$, and, similarly to section [2.8, an intertwining operator $\mathcal{A}\left(M_{1}: M^{\prime}, \mathcal{V}, \nu^{\prime}\right)$. Setting $\mathcal{V}=$ $X_{M_{1}}(M, \sigma, \operatorname{Re} \nu)$ and $\nu^{\prime}=\operatorname{Im} \nu$, we find that this operator is invertible.

$$
X(M, \sigma, \nu) \cong I\left(M_{1}, X_{M_{1}}(M, \sigma, \operatorname{Re} \nu) \otimes \mathbb{C}_{I m \nu}\right) .
$$

Now, $X(M, \sigma, \nu)$ maps onto $L(M, \sigma, \nu)$ via the operator $\mathcal{A}(M, \sigma, \nu)$ in (2.8.2). On the other hand, $X_{M_{1}}(M, \sigma, R e \nu)$ maps onto $\sigma_{1}$ by the operator $\mathcal{A}_{M_{1}}(M, \sigma, \operatorname{Re} \nu)$, and by induction (which is an exact functor) we find a map from the right-hand side of (2.9.2) onto $I\left(M_{1},\left(\sigma_{1} \otimes \mathbb{C}_{I m \nu}\right)\right)$ whose kernel is $\mathbb{H} \otimes_{\mathbb{H}_{M_{1}}}\left(\operatorname{ker} \mathcal{A}_{M_{1}}(M, \sigma, \operatorname{Re} \nu)\right) \cong$ $\operatorname{ker} \mathcal{A}\left(M^{\prime}, \sigma, \nu\right)$, which is identical to $\operatorname{ker} \mathcal{A}(M, \sigma, \nu)$.

Corollary. Assuming the previous notation, $L(M, \sigma, \nu)$ is unitary if and only if $L_{M_{1}}(M, \sigma$, Re $\nu)$ is unitary.

Proof. This follows immediately from proposition 2.9. and the fact that if $\chi$ is a purely imaginary character and $I\left(M, U \otimes \mathbb{C}_{\chi}\right)$ is irreducible, then $I\left(M, U \otimes \mathbb{C}_{\chi}\right)$ is unitary for $\mathbb{H}$ if and only if $U$ is unitary for $\mathbb{H}_{M}$. 


\section{Geometric Hecke algebras}

We will restrict to the case of geometric Hecke algebras, as in [Lu2] Lu4]. We review some facts about these algebras and their geometric classification.

3.1. For an algebraic group $\mathbf{G}$ and a $\mathbf{G}$-variety $X$, let $H_{\mathbf{G}}^{\bullet}(X)=H_{\mathbf{G}}^{\bullet}(X, \mathbb{C})$, respectively $H_{\bullet}^{\mathbf{G}}(X)=H_{\bullet}^{\mathbf{G}}(X, \mathbb{C})$ denote the equivariant cohomology, respectively homology (as in section 1 of [Lu2]). The component group of $\mathbf{G}, A(\mathbf{G})=\mathbf{G} / \mathbf{G}^{0}$ acts naturally on $H_{\mathbf{G}^{0}}^{\bullet}(X)$ and $H_{\mathbf{G}^{0}}(X)$. The cup product defines a structure of graded $H_{\mathbf{G}}^{\bullet}(X)$-module on $H_{\bullet}^{\mathbf{G}}(X)$. If $p t$ is a point of $X$, one uses the notation $H_{\mathbf{G}}^{\bullet}=H_{\mathbf{G}}^{\bullet}(\{p t\})$, respectively $H_{\bullet}^{\mathbf{G}}=H_{\bullet}^{\mathbf{G}}(\{p t\})$. There is a $\mathbb{C}$-algebra homomorphism $H_{\mathbf{G}}^{\bullet} \rightarrow H_{\mathbf{G}}^{\bullet}(X)$ induced by the map $X \rightarrow\{p t\}$, and therefore $H_{\mathbf{G}}^{\bullet}$ and $H_{\bullet}^{\mathbf{G}}$ can both be considered as $H_{\mathbf{G}^{\bullet}}$-modules.

For a subset $\mathcal{S}$ of $\mathbf{G}$, or $\mathfrak{g}$, let $Z_{\mathbf{G}}(\mathcal{S}), N_{\mathbf{G}}(\mathcal{S})$ denote the centralizer, respectively the normalizer of $\mathcal{S}$ in $\mathbf{G}$.

Let $G$ be a reductive connected complex algebraic group, with Lie algebra $\mathfrak{g}$. Let $P=L N$ denote a parabolic subgroup, with $\mathfrak{p}=\mathfrak{l}+\mathfrak{n}$ the corresponding Lie algebras, such that $\mathfrak{l}$ admits an irreducible $L$-equivariant cuspidal local system (as in [Lu2, Lu5] $\mathcal{L}$ on a nilpotent $L$-orbit $\mathcal{C} \subset \mathfrak{l}$. The classification of cuspidal local systems can be found in Lu5. In particular, $W=N(L) / L$ is a Coxeter group.

Let $H$ be the center of $L$ with Lie algebra $\mathfrak{h}$, and let $R$ be the set of nonzero weights $\alpha$ for the $a d$-action of $\mathfrak{h}$ on $\mathfrak{g}$, and $R^{+} \subset R$ the set of weights for which the corresponding weight space $\mathfrak{g}_{\alpha} \subset \mathfrak{n}$. For each parabolic $P_{j}=L_{j} N_{j}, j=1, n$, such that $P \subset P_{j}$ maximally and $L \subset L_{j}$, let $R_{j}^{+}=\left\{\alpha \in R^{+}: \alpha\left(\mathfrak{z}\left(\mathfrak{l}_{j}\right)\right)=0\right\}$, where $\mathfrak{z}\left(\mathfrak{l}_{j}\right)$ denotes the center of $\mathfrak{l}_{j}$. It is shown in Lu2 that each $R_{j}^{+}$contains a unique $\alpha_{j}$ such that $\alpha_{j} \notin 2 R$.

Let $Z_{G}(\mathcal{C})$ denote the centralizer in $G$ of a Lie triple for $\mathcal{C}$, and $\mathfrak{z}(\mathcal{C})$ its Lie algebra.

Proposition ([Lu2]). (a) $R$ is a (possibly nonreduced) root system in $\mathfrak{h}^{*}$, with simple roots $\Pi=\left\{\alpha_{1}, \ldots, \alpha_{n}\right\}$. Moreover, $W$ is the corresponding Weyl group.

(b) $H$ is a maximal torus in $Z^{0}=Z_{G}^{0}(\mathcal{C})$.

(c) $W$ is isomorphic to $W\left(Z_{G}^{0}(\mathcal{C})\right)=N_{Z^{0}}(H) / H$.

(d) The set of roots in $\mathfrak{z}(\mathcal{C})$ with respect to $\mathfrak{h}$ is exactly the set of reduced roots in $R$.

For each $j=1, n$, let $c_{j} \geq 2$ be such that

$$
\operatorname{ad}(e)^{c_{j}-2}: \mathfrak{l}_{j} \cap \mathfrak{n} \rightarrow \mathfrak{l}_{j} \cap \mathfrak{n} \neq 0, \text { and } \operatorname{ad}(e)^{c_{j}-1}: \mathfrak{l}_{j} \cap \mathfrak{n} \rightarrow \mathfrak{l}_{j} \cap \mathfrak{n}=0 .
$$

By proposition 2.12 in $\mathrm{Lu2}, c_{i}=c_{j}$ whenever $\alpha_{i}$ and $\alpha_{j}$ are $W$-conjugate. Therefore, one can define a Hecke algebra $\mathbb{H}$ as in (2.2.1), (2.2.2). In view of the proposition above, one can think of this algebra as essentially a graded Hecke algebra with unequal parameters for the centralizer $\mathfrak{z}(\mathcal{C})$. The explicit algebras which may appear are listed in 2.13 of [Lu2]. The more familiar case of Hecke algebras with equal parameters arise when one takes $P$ to be a Borel subgroup, and $\mathcal{C}$ and $\mathcal{L}$ to be trivial.

The geometric realization of $\mathbb{H}$ is obtained as follows. Consider the varieties

$$
\begin{aligned}
& \dot{\mathfrak{g}}_{N}=\left\{(x, g P) \in \mathfrak{g} \times G / P \mid A d\left(g^{-1}\right) x \in \mathfrak{n}\right\}, \\
& \ddot{\mathfrak{g}}_{N}=\left\{\left(x, g P, g^{\prime} P\right) \in \mathfrak{g} \times G / P \times G / P \mid(x, g P),\left(x, g^{\prime} P\right) \in \dot{\mathfrak{g}}_{N}\right\} .
\end{aligned}
$$


Clearly, $\ddot{\mathfrak{g}}_{N} \subset \dot{\mathfrak{g}}_{N} \times \dot{\mathfrak{g}}_{N}$. The local system $\mathcal{L}$ gives a local systems $\dot{\mathcal{L}}$ on $\dot{\mathfrak{g}}_{N}$ and $\ddot{\mathcal{L}}$ on $\ddot{\mathfrak{g}}_{N}$. When $P$ is a Borel subgroup and $\mathcal{L}$ is trivial, one recovers the classical objects: $\dot{\mathfrak{g}}_{N}$ is the cotangent bundle of the flag variety of $G$, and $\ddot{\mathfrak{g}}_{N}$ is the Steinberg variety.

The group $G \times \mathbb{C}^{*}$ acts on $\mathfrak{g}$ by $\left(g_{1}, \lambda\right) \cdot x=\lambda^{-2} A d\left(g_{1}\right) x$, for every $x \in \mathfrak{g}, g_{1} \in$ $G, \lambda \in \mathbb{C}^{*}$. In Lu2, the vector space $H_{\bullet}^{G \times \mathbb{C}^{*}}\left(\ddot{\mathfrak{g}}_{N}, \ddot{\mathcal{L}}\right)$ is endowed with left and right actions of $W$ and $S\left(\mathfrak{h}^{*} \oplus \mathbb{C}\right) \cong H_{G \times \mathbb{C}^{*}}^{\bullet}\left(\dot{\mathfrak{g}}_{N}\right)$, and it is proved (theorem 6.3 and corollary 6.4) that

$$
H_{\bullet}^{G \times \mathbb{C}^{*}}\left(\ddot{\mathfrak{g}}_{N}, \ddot{\mathcal{L}}\right) \cong \mathbb{H}, \text { as } \mathbb{H} \text {-bimodules. }
$$

3.2. Now we recall the construction of standard modules for $\mathbb{H}$. Fix a nilpotent element $e$ in $\mathfrak{g}$, and let $\mathcal{P}_{e}$ be the variety

$$
\mathcal{P}_{e}=\left\{g P \in G / P: A d\left(g^{-1}\right) e \in \mathcal{C}+\mathfrak{n}\right\} .
$$

The centralizer $Z_{G \times \mathbb{C}^{*}}(e)$ acts on $\mathcal{P}_{e}$ by $\left(g_{1}, \lambda\right) . g P=\left(g_{1} g\right) P$.

Lu2 constructs actions of $W$ and $S\left(\mathfrak{h}^{*} \oplus \mathbb{C}\right)$ on $H_{\bullet}^{Z_{G \times \mathbb{C}^{*}}^{0}(e)}\left(\mathcal{P}_{e}, \dot{\mathcal{L}}\right)$, and proves that these are compatible with the relations between the generators of $\mathbb{H}$, therefore obtaining a module of $\mathbb{H}$ (theorem 8.13). The component group $A_{G \times \mathbb{C}^{*}}(e)$ acts on $H_{\bullet}^{Z_{G \times \mathbb{C}^{*}}^{0}(e)}\left(\mathcal{P}_{e}, \dot{\mathcal{L}}\right)$, and commutes with the $\mathbb{H}$-action $(8.5)$.

Consider the variety $\mathcal{V}$ of semisimple $Z_{G \times \mathbb{C}^{*}}^{0}(e)$-orbits on the Lie algebra $\mathfrak{z} G \times \mathbb{C}^{*}(e)$ $=\left\{\left(x, r_{0}\right) \in \mathfrak{g} \oplus \mathbb{C}:[x, e]=2 r_{0} e\right\}$ of $Z_{G \times \mathbb{C}^{*}}(e)$. The affine variety $\mathcal{V}$ has $H_{Z_{G \times \mathbb{C}^{*}}^{\bullet}(e)}^{\bullet}$ as the coordinate ring. Define the $\mathbb{H}$-modules

$$
X\left(s, r_{0}, e\right)=\mathbb{C}_{\left(s, r_{0}\right)} \otimes_{H_{Z_{G \times \mathbb{C}^{*}}^{0}(e)}^{\bullet}} H_{\bullet}^{Z_{G \times \mathbb{C}^{*}}^{0}(e)}\left(\mathcal{P}_{e}, \dot{\mathcal{L}}\right),
$$

where $\mathbb{C}_{\left(s, r_{0}\right)}$ denotes the $H_{Z_{G \times \mathbb{C}^{*}}}^{\bullet}(e)$-module given by the evaluation at $\left(s, r_{0}\right) \in \mathcal{V}$, $H_{Z_{G \times \mathbb{C}^{*}}^{\bullet}(e)}^{\bullet} \rightarrow \mathbb{C}$.

Let $A_{G \times \mathbb{C}^{*}}\left(e, s, r_{0}\right)$ denote the stabilizer of $\left(s, r_{0}\right)$ in $A_{G \times \mathbb{C}^{*}}(e)$. For each $\psi \in$ $\left.A_{G \times \mathbb{C}^{*}(e, s}, r_{0}\right)$, define

$$
X\left(s, r_{0}, e, \psi\right)=\operatorname{Hom}_{A_{G \times \mathbb{C}^{*}}\left(e, s, r_{0}\right)}\left[\psi: X\left(s, r_{0}, e\right)\right] .
$$

In particular, when $\left(s, r_{0}\right)=\mathbf{0}$, we have $(7.2,8.9$ in Lu2 and $10.12(\mathrm{~d})$ in [Lu3]

$$
X(\mathbf{0}, e) \cong H_{\bullet}^{\{1\}}\left(\mathcal{P}_{e}, \dot{\mathcal{L}}\right) \text { as } W \times A_{G}(e) \text {-modules. }
$$

Let $\widehat{A_{G}(e)^{0}}$ and $\widehat{A_{G}(e, s)^{0}}$ denote the set of representations $\psi$ which appear in the $A_{G}(e)$-module $H_{\bullet}^{\{1\}}\left(\mathcal{P}_{e}, \dot{\mathcal{L}}\right)$, respectively in the restriction of this module to $A_{G}(e, s)$.

Theorem ([Lu2,, $\mathrm{Lu} 3])$. Assume $r_{0} \neq 0$.

(a) $\left([\mathrm{Lu} 2,8.10) X\left(s, r_{0}, e, \psi\right) \neq 0 \text { if and only if } \psi \in A_{G} \widehat{\left(e, s, r_{0}\right.}\right)^{0}$.

(b) $(\underline{\mathrm{Lu} 2}, \mathrm{8} .15)$ Any simple $\mathbb{H}$-module on which $r$ acts by $r_{0}$ is a quotient $\bar{X}\left(s, r_{0}\right.$, $e, \psi)$ of an $X\left(s, r_{0}, e, \psi\right)$, where $\left.\psi \in \widehat{A_{G}(e, s}\right)^{0}$.

(c) ([Lu3], 8.18) The set of isomorphism classes of simple $\mathbb{H}$-modules with central character $\left(s, r_{0}\right)$ is in one-to-one correspondence with the set

$$
\left.\mathcal{M}_{s, r_{0}}=Z_{G}(s) \text {-conjugacy classes on }\left\{(e, \psi):[s, e]=2 r_{0} e, \psi \in \widehat{A_{G}(e, s}\right)^{0}\right\} \text {. }
$$


3.3. Fix a semisimple class $\left(s, r_{0}\right) \in \mathcal{V}$. The following theorem is immediately implied by the results in [Lu3], 10.5-10.7.

Theorem ([u3]). If a composition factor $Y$ of the standard module $X\left(s, r_{0}, e, \psi\right)$ is parameterized by the class $\left(e^{\prime}, \psi^{\prime}\right) \in \mathcal{M}_{s, r_{0}}$, then necessarily $\mathcal{O} \subset \overline{\mathcal{O}^{\prime}}$, where $\mathcal{O}$, $\mathcal{O}^{\prime}$ are the $Z_{G}(s)$-orbits of e, respectively $e^{\prime}$ in $\mathcal{M}_{s, r_{0}}$. Moreover, $\mathcal{O}=\mathcal{O}^{\prime}$ if and only if $Y=\bar{X}\left(s, r_{0}, e, \psi\right)$.

This is the geometric equivalent of the classical results for real and $p$-adic groups concerning the minimality of the Langlands parameter in a standard module (see BW, IV.4.13 and XI.2.13).

Finally, an immediate corollary of theorem 3.3 (see 8.17 in Lu2, equivalently 10.9 in [Lu3]) is that, if $\mathcal{O}$ is the unique open $Z_{G}(s)$-orbit in $\mathcal{M}_{s, r_{0}}$, then $X\left(s, r_{0}, e, \psi\right)$, $\psi \in \widehat{A_{G}(e, s)^{0}}$ is simple.

3.4. An important role in the determination of the unitary dual will be played by the $W$-structure of standard and simple modules.

The continuation argument in 10.13 in Lu3 in conjunction with (3.2.4) shows that

$$
\left.X\left(s, r_{0}, e\right)\right|_{W} \cong H_{\bullet}^{\{1\}}(\mathcal{P}, \dot{\mathcal{L}}), \text { as } W \times A_{G}(s, e) \text {-representations. }
$$

(By definition, $H_{j}^{\{1\}}(X, \mathcal{L})=H_{c}^{2 \operatorname{dim}(X)-j}\left(X, \mathcal{L}^{*}\right)^{*}$, where $H_{c}^{\bullet}$ denotes the cohomology with compact support, while ${ }^{*}$ denotes the dual vector space or local system.)

Denote

(3.4.2) $\mathcal{N}_{G}=G$-conjugacy classes of pairs $\left\{(e, \phi): e \in \mathfrak{g}\right.$ nilpotent, $\left.\phi \in \widehat{A_{G}(e)}\right\}$.

The generalized Springer correspondence ([Lu5]) gives, in particular, an injection

$$
\Phi_{\mathcal{C}, \mathcal{L}}: \widehat{W} \hookrightarrow \mathcal{N}_{G} .
$$

(Recall that $W=N_{G}(L) / L$.) The case of the classical Springer correspondence appears when $P$ is a Borel subgroup, $\mathcal{C}$ and $\mathcal{L}$ are trivial, and $W$ is the Weyl group of $G$.

More precisely, let $(e, \phi)$ be a $G$-conjugacy class in $\mathcal{N}_{G}$. Set $\mathcal{O}_{e}=G \cdot e$, and let $\varepsilon_{\phi}$ be the local system on $\mathcal{O}_{e}$ corresponding to $\phi$. Then [Lu5] $(6.2,6.3)$ attaches to $(e, \phi)$ a unique $G$-conjugacy class $\left(L^{\prime}, \mathcal{C}^{\prime}, \mathcal{L}^{\prime}\right)$, where $L^{\prime} \subset P^{\prime}$ is a Levi subgroup, $\mathcal{C}^{\prime}$ a nilpotent $L^{\prime}$-orbit in $\mathfrak{l}^{\prime}$, and $\mathcal{L}^{\prime}$ is a local system on $\mathcal{C}^{\prime}$, such that:

$$
\begin{aligned}
& \text { (a) } H_{c}^{\operatorname{dim}\left(\mathcal{O}_{e}\right)-\operatorname{dim}\left(\mathcal{C}^{\prime}\right)}\left(\mathcal{O}_{e} \cap\left(\mathcal{C}^{\prime}+\mathfrak{n}^{\prime}\right), \varepsilon_{\phi}\right) \neq 0 \text {; } \\
& \text { (b) } P^{\prime} \text { is minimal with respect to }(a) .
\end{aligned}
$$

The local system $\mathcal{L}^{\prime}$ on $\mathcal{C}^{\prime}$ is constructed from $\varepsilon_{\phi}$ (see [Lu5], 6.2, for the precise definition). (Note that in fact, (3.4.4) gives a definition of cuspidal: one could define $\mathcal{L}^{\prime}$ to be a cuspidal local system for $G$ if in (3.4.4), $P^{\prime}=G$.) It is shown in [Lu5] that all $\mathcal{L}^{\prime}$ appearing in this way must be cuspidal for the corresponding $L^{\prime}$.

If we denote by $\mathcal{M}_{\mathcal{C}, \mathcal{L}}$ the subset of $\mathcal{N}_{G}$ attached to $(L, \mathcal{C}, \mathcal{L})$ by (3.4.4), the generalized Springer correspondence (3.4.3) can be reformulated as a bijection $\mathcal{M}_{\mathcal{C}, \mathcal{L}} \leftrightarrow \widehat{W}$. For $(e, \phi) \in \mathcal{M}_{\mathcal{C}, \mathcal{L}}$, there corresponds an irreducible $W$-representation, which we will denote by $\mu\left(\mathcal{O}_{e}, \phi\right)$, constructed in $\operatorname{Hom}_{A(e)}\left[\phi: H_{c}^{\bullet}\left(\mathcal{P}_{e}, \dot{\mathcal{L}}\right)\right]$.

With respect to the closure ordering of nilpotent orbits, the smallest orbit, $\mathcal{O}_{\text {min }}$, appearing in $\operatorname{Im} \Phi_{\mathcal{C}, \mathcal{L}}$ is $G \cdot \mathcal{C}$, and the largest orbit, $\mathcal{O}_{\max }$, is the Lusztig-Spaltenstein induced orbit $\operatorname{Ind}_{L}^{G}(\mathcal{C})$. Moreover, each one of these two orbits supports exactly one 
local system (denoted $\phi_{\max }$ and $\phi_{\min }$, respectively) which enters the parameterization of $\mathbb{H}$.

The correspondence is normalized such that

$$
\mu\left(\mathcal{O}_{\text {min }}, \phi_{\text {min }}\right)=\text { triv and } \mu\left(\mathcal{O}_{\max }, \phi_{\max }\right)=\text { sgn } .
$$

3.5. The previous discussion gives a classification of simple $\mathbb{H}$-modules via lowest $W$-types. This is the analogue of Vogan's classification by lowest $K$-types for real reductive groups ( $\left.\mathrm{V}_{02}\right)$, and it appeared in the setting of equal parameter affine Hecke algebras in BM1.

Fix $\psi \in \widehat{A_{G}(s, e)}$. If $\phi \in \widehat{A_{G}(e)^{0}}$ and

$$
\operatorname{Hom}_{W}\left[\left(\mu\left(\mathcal{O}_{e}, \phi\right): X\left(s, r_{0}, e, \psi\right)\right] \neq 0,\right.
$$

then we will call $\mu(\mathcal{O}, \phi)$ a lowest $W$-type for $X\left(s, r_{0}, e, \psi\right)$.

Proposition. The simple module $\bar{X}\left(s, r_{0}, e, \psi\right)$ is the unique composition factor of $X\left(s, r_{0}, e, \psi\right)$ which contains the lowest $W$-types $\mu\left(\mathcal{O}_{e}, \phi\right)$ with multiplicity $\left[\left.\phi\right|_{A_{G}(s, e)}: \psi\right]$. Moreover, if $\mu^{\prime}$ is a $W$-type appearing in $\bar{X}\left(s, r_{0}, e, \psi\right)$, different than the lowest $W$-types, then $\Phi_{\mathcal{C}, \mathcal{L}}\left(\mu^{\prime}\right)=\left(\mathcal{O}^{\prime}, \phi^{\prime}\right)$, for some $\mathcal{O}^{\prime}$ such that $\mathcal{O} \subset \overline{\mathcal{O}^{\prime}} \backslash \mathcal{O}^{\prime}$

Proof. It follows directly from theorem 3.3 and the discussion in section 3.4, particularly, equation (3.4.1).

3.6. If $s \in \mathfrak{g}$ is semisimple, let $s=s_{\text {hyp }}+s_{\text {ell }}$ denote its decomposition into hyperbolic and elliptic parts.

Theorem ([Lu4). A simple $\mathbb{H}$-module $\bar{X}\left(s, r_{0}, e, \psi\right)$ is tempered if and only if $\left\{s_{\text {hyp }}, e\right\}$ can be embedded into a Lie triple of $\mathcal{O}_{e}$. In this case $\bar{X}\left(s, r_{0}, e, \psi\right)=$ $X\left(s, r_{0}, e, \psi\right)$.

If, in addition, $\mathcal{O}_{e}$ is a distinguished nilpotent orbit, then $X\left(s, r_{0}, e, \psi\right)$ is a discrete series.

Note that, if $(s, e, \phi)$ is the geometric parameter of a real $\left(s_{e l l}=0\right)$ tempered simple $\mathbb{H}$-module, $A_{G}(s, e)=A_{G}(e)$, and $X\left(s, r_{0}, e, \phi\right)$ has a unique lowest $W$-type, namely $\mu\left(\mathcal{O}_{e}, \phi\right)$. In addition, $\mu\left(\mathcal{O}_{e}, \phi\right)$ has multiplicity one. We need one more fact, in the notation before (3.4.4):

$H_{c}^{\operatorname{dim}\left(\mathcal{O}_{e}\right)-\operatorname{dim}\left(\mathcal{C}^{\prime}\right)}\left(\mathcal{O}_{e} \cap\left(\mathcal{C}^{\prime}+\mathfrak{n}^{\prime}\right), \varepsilon_{\phi}\right) \neq 0$ if and only if $H_{c}^{\bullet}\left(\mathcal{O}_{e} \cap\left(\mathcal{C}^{\prime}+\mathfrak{n}^{\prime}\right), \varepsilon_{\phi}\right) \neq 0$.

For Borel-Moore homology and the classical Springer correspondence, this fact was proved first in [Sh1] (theorem 2.5) for classical groups and [Sh2] for $F_{4}$, [BS] (section 2 ) for $E_{6}, E_{7}, E_{8}$, and for $G_{2}$ it follows from Spr2 (7.16). In the generality needed here, it is proved in [Lu7, theorem 24.4(d). The proof is a consequence of the algorithm for computing Green functions.

Therefore, we have a bijection between $\widehat{W}$ and simple tempered $\mathbb{H}$-modules with real central character, when $\mathbb{H}$ is of geometric type. Combining this with proposition 3.5. we obtain the following result.

Corollary. Assume $\mathbb{H}$ is of geometric type. The set of simple tempered $\mathbb{H}$-modules with real central character is linearly independent in the Grothendieck group of $W$. 
Remark. In the Grothendieck group of $W$, one can consider two sets: one set given by the $W$-characters of tempered $\mathbb{H}$-modules with real central character, and the other set, the natural basis of irreducible $W$-representations. The statements above say that the first set is also a basis, and that, in some ordering (coming from the lowest $W$-type map), the change of bases matrix is unitriangular. It is natural to expect that this should hold for arbitrary parameters Hecke algebras $\mathbb{H}$. For $\mathbb{H}$ of type $G_{2}$ with arbitrary parameters, this correspondence is part of $\mathrm{KR}$. When $\mathbb{H}$ is of type $B / C$, the cases not covered here are all accounted for by the recent paper $\mathrm{Ka}$ and the geometry of the "exotic nilpotent cone". Since a geometric framework exists, this result will most likely follow, but the "exotic" lowest $W$ type correspondence may not be compatible with the one constructed for Lusztig's parameters. For multi-laced Hecke algebras with unequal parameters (including $F_{4}$ ), the results of $\mathrm{OS}$ may also imply this statement.

\section{Hermitian FORMS}

We retain the notation from the previous sections. The graded Hecke algebra $\mathbb{H}$ will be assumed geometric and all parameters and central characters are assumed to be real. We will fix $r_{0}=1 / 2$ and drop it from the notation. (It is sufficient to determine the unitary dual of $\mathbb{H}$ for one particular value $r_{0} \neq 0$.)

4.1. Let $(M, \sigma, \nu), \nu$ real, be a Hermitian Langlands parameter as in section 2.8 , We constructed the intertwining operator

$$
\mathcal{A}(M, \sigma, \nu): X(M, \sigma, \nu) \rightarrow I\left(M, \sigma \otimes \mathbb{C}_{-\nu}\right), x \otimes\left(v \otimes 1_{\nu}\right) \mapsto x r_{w} \otimes\left(a_{w}(v) \otimes 1_{-\nu}\right),
$$

where $w$ is such that $w M=M, a_{w}: w \sigma \stackrel{\cong}{\longrightarrow} \sigma$, and $w \nu=-\nu$.

By the results in the previous section, $X(M, \sigma, \nu)$ contains a special set of $W$ representations, the lowest $W$-types. It is an empirical fact that every $L(M, \sigma, \nu)$ contains one lowest $W$-type $\mu_{0}$ with multiplicity 1 . (This is verified case by case for the exceptional Hecke algebra; for those of classical types this is automatic from the fact that the component groups $A_{G}(e)$ of nilpotent elements $e$ are always abelian.)

As a $\mathbb{C}[W]$-module,

$$
\left.I\left(M, \sigma \otimes \mathbb{C}_{\nu}\right)\right|_{W}=\mathbb{C}[W] \otimes_{\mathbb{C}[W(M)]}\left(\left.\sigma\right|_{W(M)}\right) .
$$

For any $W$-type $\left(\mu, V_{\mu}\right), \mathcal{A}(M, \sigma, \nu)$ induces an operator

$$
r_{\mu}(M, \sigma, \nu): \operatorname{Hom}_{W}\left(\mu, \mathbb{C}[W] \otimes_{\mathbb{C}[W(M)]} \sigma\right) \rightarrow \operatorname{Hom}_{W}\left(\mu, \mathbb{C}[W] \otimes_{\mathbb{C}[W(M)]} \sigma\right) .
$$

By Frobenius reciprocity,

$$
\operatorname{Hom}_{W}\left(\mu, \mathbb{C}[W] \otimes_{\mathbb{C}[W(M)]} \sigma\right) \cong \operatorname{Hom}_{W(M)}(\mu, \sigma) .
$$

In conclusion, $\mathcal{A}(M, \sigma, \nu)$ gives rise to operators

$$
r_{\mu}(M, \sigma, \nu): \operatorname{Hom}_{\mathbb{C}[W(M)]}(\mu, \sigma) \rightarrow \operatorname{Hom}_{\mathbb{C}[W(M)]}(\mu, \sigma) .
$$

The operator $r_{\mu_{0}}(M, \sigma, \nu)$ is a scalar, and we normalize the intertwining operator $\mathcal{A}(M, \sigma, \nu)$ so that this scalar is 1 .

Recall the map $\epsilon: \mathbb{H} \rightarrow \mathbb{A}$ (section 2.8). We denote by $\epsilon(x)(\nu)$, the evaluation of an element $\epsilon(x) \in \mathbb{A}=S\left(\mathfrak{h}^{*}\right)$ at $\nu \in \mathfrak{h}$.

Theorem ([BM3]). Let $(M, \sigma, \nu)$ be a Hermitian Langlands parameter.

(1) The map $\mathcal{A}(M, \sigma, \nu)$ is an intertwining operator. 
(2) The image of the operator $\mathcal{A}(M, \sigma, \nu)$ is $L(M, \sigma, \nu)^{h}$ and the Hermitian form on $L(M, \sigma, \nu)$ is given by:

$$
\begin{aligned}
\left\langle t_{x} \otimes\left(v_{x} \otimes 1_{\nu}\right), t_{y} \otimes\left(v_{y} \otimes 1_{\nu}\right)\right\rangle & =\left\langle t_{x} \otimes\left(v_{x} \otimes 1_{\nu}\right), t_{y} r_{w_{m}} \otimes\left(a_{m}\left(v_{y}\right) \otimes 1_{-\nu}\right)\right\rangle_{h}, \\
& =\left\langle\epsilon\left(t_{y}^{*} t_{x} r_{w_{m}}\right)(\nu) a_{m}\left(v_{x}\right), v_{y}\right\rangle_{M} .
\end{aligned}
$$

The discussion in this section can be summarized in the following corollary.

Corollary. A Langlands parameter $(M, \sigma, \nu), \nu$ real, is unitary if and only if the following two conditions are satisfied:

(1) $w_{m} M=M, w_{m} V \cong V, w_{m} \nu=-\nu$;

(2) the normalized operators $r_{\mu}(M, \sigma, \nu)$ are positive semidefinite for all $\mu \in \widehat{W}$, such that $\operatorname{Hom}_{W(M)}[\mu: V] \neq 0$.

One of the main tools for showing $\mathbb{H}$-modules are not unitary is to compute the signature of certain $r_{\mu}(M, \sigma, \nu)$.

4.2. We consider now the particular case of spherical modules.

Definition. An $\mathbb{H}$-module $V$ is called spherical if $\operatorname{Hom}_{W}[V$,triv $] \neq 0$. It is called generic if $\operatorname{Hom}_{W}[V, s g n] \neq 0$.

In the case of Hecke algebra with equal parameters, these definitions are motivated by the correspondence with the representations of split $p$-adic groups with Iwahori fixed vectors ( BM4, Re2]). In that case, generic $\mathbb{H}$-modules correspond to representations of the $p$-adic group admitting Whittaker models, and spherical $\mathbb{H}$-modules to representations having fixed vectors under a hyperspecial maximal compact open subgroup. We emphasize that for a split adjoint $p$-adic group, the unipotent representations admitting Whittaker models have necessarily Iwahori fixed vectors ( $\mathrm{Re} 2$ ).

The Iwahori-Matsumoto involution, $I M$, defined by

$$
\begin{aligned}
& I M\left(t_{w}\right):=(-1)^{l(w)} t_{w}, \\
& I M(\omega):=-\omega, \quad \omega \in \mathfrak{h}^{*},
\end{aligned}
$$

interchanges spherical and generic modules. From (2.4.1), it follows that $I M$ commutes with the *-operation on $\mathbb{H}$. From (2.4.2), it follows immediately then that $I M$ preserves Hermitian and unitary modules.

Let $X(\nu)=\mathbb{H} \otimes_{\mathbb{A}} \mathbb{C}_{\nu}, \nu \in \mathfrak{h}_{\mathbb{R}}$, be a principal series module. Clearly, $X(\nu) \cong$ $\mathbb{C}[W]$, as $W$-modules, and in particular, $X(\nu)$ has a unique irreducible subquotient containing the triv representation of $W$. Denote this by $L(\nu)$. Let $\mathfrak{h}_{\mathbb{R}}^{+}$denote the dominant parameters in $\mathfrak{h}_{\mathbb{R}}$. The Langlands classification and the considerations about intertwining operators presented in the previous sections are summarized as follows.

Proposition. Assume $\nu \in \mathfrak{h}_{\mathbb{R}}^{+}$.

(1) The principal series $X(\nu)$ has a unique irreducible quotient, $L(\nu)$, which is spherical. Moreover, every spherical $\mathbb{H}$-module appears in this way.

(2) A spherical module $L(\nu)$ is Hermitian if and only if $w_{0} \nu=-\nu$.

(3) If $w_{0} \nu=-\nu$, the image of the intertwining operator

$$
A(\nu): X(\nu) \rightarrow X(-\nu), A(\nu)\left(x \otimes 1_{\nu}\right)=x r_{w_{0}} \otimes 1_{-\nu},
$$

normalized so that it is +1 on the spherical vector, is $L(-\nu)$. 
In particular, $X(\nu)$ is reducible if and only if $\langle\alpha, \nu\rangle=c_{\alpha}$, for some $\alpha \in R^{+}$. For every $\mu \in \widehat{W}$, let

$$
r_{\mu}(\nu): \mu^{*} \rightarrow \mu^{*}
$$

be the operator defined by (4.1.5). Let $w_{0}=s_{1} \cdots s_{k}$ be a reduced decomposition of $w_{0}\left(k=\left|R^{+}\right|\right)$, where $s_{j}=s_{\alpha_{j}}, \alpha_{j} \in \Pi$. The operator $r_{\mu}(\nu)$ has a decomposition

$$
r_{\mu}(\nu)=r_{\mu, s_{1}}\left(s_{2} \cdots s_{k} \nu\right) \cdot r_{\mu, s_{2}}\left(s_{3} \cdots s_{k} \nu\right) \cdots r_{\mu, s_{k}}(\nu),
$$

where each $r_{\mu, s_{j}}\left(\nu^{\prime}\right)$ is determined by the equation

$$
r_{\mu, s_{j}}\left(\nu^{\prime}\right)=\left\{\begin{array}{cl}
1, & \text { on the }(+1) \text {-eigenspace of } s_{j} \text { on } \mu^{*} \\
\frac{c_{\alpha_{j}}-\nu^{\prime}}{c_{\alpha_{j}}+\nu^{\prime}}, & \text { on the }(-1) \text {-eigenspace of } s_{j} \text { on } \mu^{*}
\end{array}\right.
$$

4.3. As in corollary 4.1 a spherical Hermitian module $L(\nu)$ is unitary if and only if $r_{\mu}(\nu)$ are positive semidefinite for all $\mu \in \widehat{W}$.

Definition. Define the 0-complementary series to be the set $\left\{\nu \in \mathfrak{h}_{\mathbb{R}}^{+}: X(\nu)=\right.$ $L(\nu)$ unitary .

The 0 -complementary series when $\mathbb{H}$ is of type $B_{n} / C_{n}$, with arbitrary unequal parameters, were determined in $\left[\mathrm{BC} 2\right.$. For type $G_{2}$, using the machinery presented in the previous section, in particular the signatures of $r_{\mu}(\nu)$, it is an easy calculation. We record the result, without proof, next. We use the simple roots

$$
\alpha_{1}=\left(\frac{2}{3},-\frac{1}{3},-\frac{1}{3}\right) \text { and } \alpha_{2}=(-1,1,0) .
$$

The Hecke algebra $\mathbb{H}\left(G_{2}\right)$ has $c_{\alpha_{1}}=1$ and $c_{\alpha_{2}}=c>0$.

Proposition. Let $\nu=\left(\nu_{1}, \nu_{1}+\nu_{2},-2 \nu_{1}-\nu_{2}\right), \nu_{1} \geq 0, \nu_{2} \geq 0$, be a dominant (spherical) parameter for $\mathbb{H}\left(G_{2}\right)$. There are nine cases depending on the parameter c. The 0-complementary series are as follows:

(1) $0<c<1:\left\{3 \nu_{1}+2 \nu_{2}<c\right\} \cup\left\{3 \nu_{1}+\nu_{2}>c, \nu_{2}<c, 2 \nu_{1}+\nu_{2}<1\right\}$.

(2) $c=1:\left\{3 \nu_{1}+2 \nu_{2}<1\right\} \cup\left\{3 \nu_{1}+\nu_{2}>1,2 \nu_{1}+\nu_{2}<1\right\}$.

(3) $1<c<\frac{3}{2}:\left\{3 \nu_{1}+2 \nu_{2}<c\right\} \cup\left\{3 \nu_{1}+\nu_{2}>c, 2 \nu_{1}+\nu_{2}<1\right\}$.

(4) $c=\frac{3}{2}:\left\{3 \nu_{1}+2 \nu_{2}<\frac{3}{2}\right\}$.

(5) $\frac{3}{2}<c<2:\left\{3 \nu_{1}+2 \nu_{2}<c, 2 \nu_{1}+\nu_{2}<1\right\}$.

(6) $c=2:\left\{2 \nu_{1}+\nu_{2}<1\right\}$.

(7) $2<c<3:\left\{2 \nu_{1}+\nu_{2}<1\right\} \cup\left\{\nu_{1}+\nu_{2}>1,3 \nu_{1}+2 \nu_{2}<c\right\}$.

(8) $c=3:\left\{2 \nu_{1}+\nu_{2}<1\right\} \cup\left\{\nu_{1}+\nu_{2}>1,3 \nu_{1}+2 \nu_{2}<3\right\}$.

(9) $c>3:\left\{2 \nu_{1}+\nu_{2}<1\right\} \cup\left\{\nu_{1}+\nu_{2}>1, \nu_{1}<1,3 \nu_{1}+2 \nu_{2}<c\right\}$.

In addition, the only other unitary spherical representations are the endpoints of the 0-complementary series, and the trivial representation, for which $\left(\nu_{1}, \nu_{2}\right)=$ $(1, c)$.

In this notation, the Hecke algebras of type $G_{2}$ which appear geometrically in Lu6 are the cases $c=1$ (equal parameters) and $c=9$. The latter corresponds to a cuspidal local system on the nilpotent $2 A_{2}$ in $E_{6}$. 
4.4. We present the unitary dual with real central character for the geometric Hecke algebra of type $G_{2}$ in more detail. In the notation as above, this is $\mathbb{H}\left(G_{2},(1,9)\right)$, but it is better for our parameterization to think of it as the isomorphic $\mathbb{H}\left(G_{2},(3,1)\right)$. The geometry is attached to the cuspidal local system on $2 A_{2}$ in $E_{6}$. The nilpotent orbits $\mathcal{O}$ below and their closure relations are as in $\mathrm{Ca}$, and the notation for $W\left(G_{2}\right)$-representations is as in $\mathrm{Al}$.

Proposition. The unitary irreducible modules with real central character for $\mathbb{H}\left(G_{2},(3,1)\right)$ are:

TABLE 1: Unitary dual for $\mathbb{H}\left(G_{2},(3,1)\right)$

\begin{tabular}{|c|c|c|c|}
\hline $\mathcal{O}$ & Central character & LWT & Unitary parameters \\
\hline $2 A_{2}$ & $\left(\nu_{1}, \nu_{1}+\nu_{2},-2 \nu_{1}-\nu_{2}\right)$ & $1_{1}$ & $\left\{0 \leq \nu_{1}, 0 \leq \nu_{2}, 3 \nu_{1}+2 \nu_{2} \leq 1\right\} \cup$ \\
& & & $\left\{0 \leq \nu_{1}, 3 \nu_{1}+\nu_{2} \geq 1, \nu_{2} \geq 1,2 \nu_{1}+\nu_{2} \leq 3\right\}$ \\
\hline $2 A_{2}+A_{1}$ & $\left(-\frac{1}{2}+\nu, \frac{1}{2}+\nu,-2 \nu\right)$ & $1_{3}$ & $\left\{0 \leq \nu \leq \frac{3}{2}\right\}$ \\
\hline$A_{5}$ & $\left(3,-\frac{3}{2}+\nu,-\frac{3}{2}-\nu\right)$ & $2_{2}$ & $D . S$. \\
\hline$E_{6}\left(a_{3}\right)$ & $(1,2,-3)$ & $2_{1}$ & $D . S$. \\
\hline$E_{6}\left(a_{1}\right)$ & $(2,3,-5)$ & $1_{4}$ & $S t$ \\
\hline$E_{6}$ & $(3,4,-7)$ & $1_{2}$ & I \\
\hline \hline
\end{tabular}

Here D.S. stands for discrete series, and $S t$ for the Steinberg module (which is a discrete series). The proof is by direct computations, similar, but much easier than those in the next section. We do not include the explicit calculations. We remark that the unitary pieces attached to the nilpotent orbits $2 A_{2}+A_{1}$ and $A_{5}$ match precisely the spherical unitary dual for the Hecke algebra of type $A_{1}$ with parameter 3, respectively 1 . The centralizers of these two orbits are both of type $A_{1}$, so this is consistent with our general philosophy.

To help visualize the answer, we include a picture of the spherical unitary dual in this case; see Figure 1 .

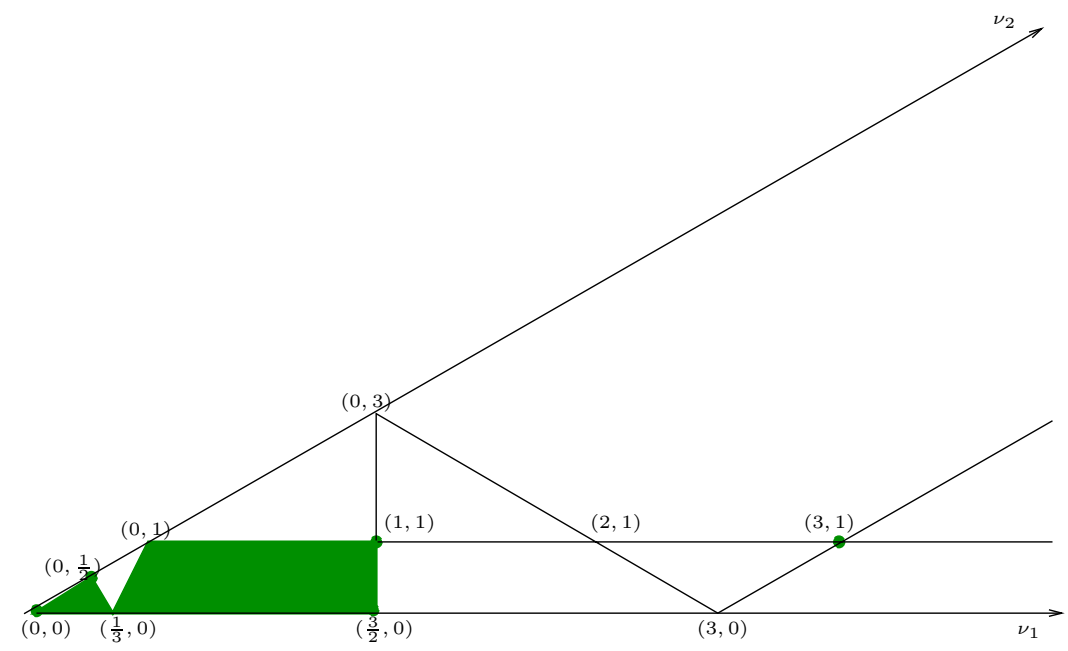

Figure 1. Spherical unitary dual for $\mathbb{H}\left(G_{2},(3,1)\right)$ 


\section{An example: Geometric Hecke algebra of type $F_{4}$}

We present the classification of the unitary dual for the Hecke algebra $\mathbb{H}$ with unequal parameters of type $F_{4}$, which appears geometrically in the classification of [Lu6]. It has labels 1 on the long roots, and 2 on the short roots. We use the following choice for the roots:

$$
\alpha_{1}=\epsilon_{1}-\epsilon_{2}-\epsilon_{3}-\epsilon_{4}, \quad \alpha_{2}=2 \epsilon_{4}, \quad \alpha_{3}=\epsilon_{3}-\epsilon_{4}, \quad \alpha_{4}=\epsilon_{2}-\epsilon_{3} .
$$

This algebra is attached to the cuspidal local system on the nilpotent orbit $\left(3 A_{1}\right)^{\prime \prime}$ in $E_{7}$. The simple modules are parameterized therefore by a subset of the nilpotent orbits in $E_{7}$, larger in the closure ordering than $\left(3 A_{1}\right)^{\prime \prime}$. We list these nilpotent orbits $\mathcal{O}$, each with the corresponding infinitesimal character in $\mathbb{H}$, the $W$-types coming from the generalized Springer correspondence (LWT), and the centralizer $\mathfrak{z}(\mathcal{O})$ in $E_{7}$. The generalized Springer correspondence for this case was computed in Spa1. The closure ordering for complex nilpotent orbits in $E_{7}$ can be found in Ca. (We mention that there is a typographical error in [Ca], the nilpotent orbits $D_{6}\left(a_{2}\right)$ and $D_{5}\left(a_{1}\right)+A_{1}$ are comparable; see Figure 2 and [Spa2.)

TABLE 2: Nilpotent orbits for $\left(3 A_{1}\right)^{\prime \prime}$ in $E_{7}$

\begin{tabular}{|c|c|c|c|}
\hline $\mathcal{O}$ & Central character & $\mathfrak{z}(\mathcal{O})$ & LWT \\
\hline$\left(3 A_{1}\right)^{\prime \prime}$ & $\left(\nu_{1}, \nu_{2}, \nu_{3}, \nu_{4}\right)$ & $F_{4}$ & $1_{1}$ \\
\hline $4 A_{1}$ & $\left(\nu_{1}, \nu_{2}, \nu_{3}, \frac{1}{2}\right)$ & $C_{3}$ & $2_{3}$ \\
\hline$A_{2}+3 A_{1}$ & $\left(\frac{1}{2},-\frac{1}{2},-\frac{1}{2}, \frac{1}{2}\right)+\nu_{1}(2,1,1,0)+\nu_{2}(1,1,0,0)$ & $G_{2}$ & $1_{3}$ \\
\hline$\left(A_{3}+A_{1}\right)^{\prime \prime}$ & $(0,0,1,-1)+\nu_{1}\left(\frac{1}{2}, \frac{1}{2}, 0,0\right)+\nu_{2}\left(\frac{1}{2},-\frac{1}{2}, 0,0\right)$ & $B_{3}$ & $4_{2}$ \\
& $+\nu_{3}\left(0,0, \frac{1}{2}, \frac{1}{2}\right)$ & & \\
\hline$A_{3}+2 A_{1}$ & $\left(\nu_{1}, 1+\nu_{2},-1+\nu_{2}, \frac{1}{2}\right)$ & $2 A_{1}$ & $8_{3}$ \\
\hline$D_{4}\left(a_{1}\right)+A_{1}$ & $\left(\nu_{1}, \nu_{2}, \frac{3}{2}, \frac{1}{2}\right)$ & $2 A_{1}$ & $9_{1}, 2_{1}$ \\
\hline$A_{3}+A_{2}+A_{1}$ & $\left(\frac{1}{2}, \frac{1}{2},-\frac{3}{2}, \frac{1}{2}\right)+\nu(2,1,1,0)$ & $A_{1}$ & $4_{4}$ \\
\hline$D_{4}+A_{1}$ & $\left(\nu_{1}, \nu_{2}, \frac{5}{2}, \frac{1}{2}\right)$ & $B_{2}$ & $9_{3}$ \\
\hline$\left(A_{5}\right)^{\prime \prime}$ & $\left(\nu_{2}+\frac{3 \nu_{1}}{2}, 2+\frac{\nu_{1}}{2}, \frac{\nu_{1}}{2},-2+\frac{\nu_{1}}{2}\right)$ & $G_{2}$ & $8_{1}$ \\
\hline$D_{5}\left(a_{1}\right)+A_{1}$ & $\left(\frac{3}{2}+\nu,-\frac{3}{2}+\nu, \frac{3}{2}, \frac{1}{2}\right)$ & $A_{1}$ & $4_{1}$ \\
\hline$A_{5}+A_{1}$ & $\left(\frac{1}{4}, \frac{7}{4},-\frac{1}{4},-\frac{9}{4}\right)+\nu\left(\frac{3}{2}, \frac{1}{2}, \frac{1}{2}, \frac{1}{2}\right)$ & $A_{1}$ & $6_{1}$ \\
\hline$D_{6}\left(a_{2}\right)$ & $\left(\nu, \frac{5}{2}, \frac{3}{2}, \frac{1}{2}\right)$ & $A_{1}$ & $16_{1}$ \\
\hline$E_{7}\left(a_{5}\right)$ & $\left(\frac{5}{2}, \frac{3}{2}, \frac{1}{2}, \frac{1}{2}\right)$ & 1 & $12_{1}, 6_{2}$ \\
\hline$D_{5}+A_{1}$ & $\left(2+\nu,-2+\nu, \frac{5}{2}, \frac{1}{2}\right)$ & $A_{1}$ & $2_{2}$ \\
\hline$D_{6}\left(a_{1}\right)$ & $\left(\nu, \frac{7}{2}, \frac{3}{2}, \frac{1}{2}\right)$ & $A_{1}$ & $9_{2}$ \\
\hline$E_{7}\left(a_{4}\right)$ & $\left(\frac{7}{2}, \frac{3}{2}, \frac{1}{2}, \frac{1}{2}\right)$ & 1 & $4_{3}, 8_{2}$ \\
\hline$D_{6}$ & $\left(\nu, \frac{9}{2}, \frac{5}{2}, \frac{1}{2}\right)$ & $A_{1}$ & $9_{4}$ \\
\hline$E_{7}\left(a_{3}\right)$ & $\left(\frac{9}{2}, \frac{5}{2}, \frac{1}{2}, \frac{1}{2}\right)$ & 1 & $8_{4}, 1_{2}$ \\
\hline$E_{7}\left(a_{2}\right)$ & $\left(\frac{11}{2}, \frac{5}{2}, \frac{3}{2}, \frac{1}{2}\right)$ & $4_{5}$ \\
\hline$E_{7}\left(a_{1}\right)$ & $\left(\frac{13}{2}, \frac{7}{2}, \frac{3}{2}, \frac{1}{2}\right)$ & 1 & 2 \\
\hline$E_{7}$ & $\left(\frac{17}{2}, \frac{9}{2}, \frac{5}{2}, \frac{1}{2}\right)$ & $1_{4}$ \\
\hline \hline
\end{tabular}




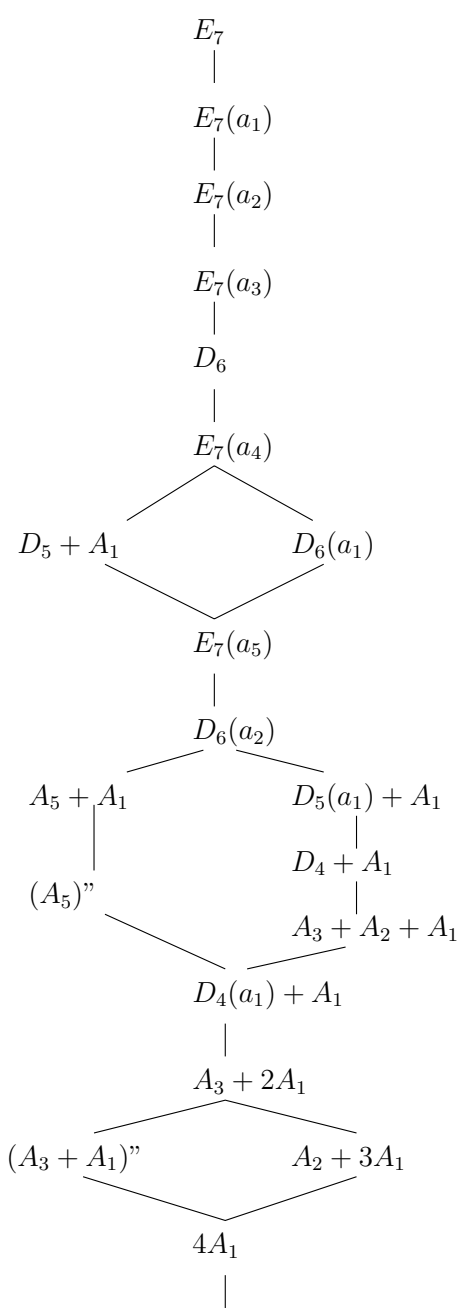

$\left(3 A_{1}\right) "$

FiguRE 2. Nilpotent orbits parameterizing the $\mathbb{H}\left(F_{4},(1,2)\right)$-modules

5.1. Results. The spherical unitary dual is listed in Table 4. These are the unitary modules parameterized by $\mathcal{O}_{\min }=\left(3 A_{1}\right)^{\prime \prime}$. For the rest of the nilpotents, the unitary sets are presented next. For every nilpotent $\mathcal{O}$ in Table 2 with $\{e, h, f\} \subset \mathcal{O}$ a Lie triple, and $\phi \in \widehat{A_{G}(e)^{0}}$, let

$$
\begin{array}{r}
\mathcal{U}(\mathcal{O}, \phi)=\left\{(\nu, \psi): s=\frac{h}{2}+\nu, \phi \in \widehat{\left.A_{G(s, e}\right)^{0}}, \operatorname{Hom}_{A(s, e)}[\psi: \phi] \neq 0,\right. \\
\bar{X}(s, e, \psi) \text { is unitary }\}
\end{array}
$$


denote the set of unitary parameters associated to $(\mathcal{O}, \phi)$. The goal is to relate $\mathcal{U}(\mathcal{O}, \phi)$ with the spherical unitary dual $\mathcal{S U}$ of a Hecke algebra of different type.

Theorem. With the notation as before (particularly Table 2 and (5.1.1)), the unitary irreducible modules with real central character for $\mathbb{H}\left(F_{4},(1,2)\right)$ are:

TABLE 3: Unitary dual of $\mathbb{H}\left(F_{4},(1,2)\right)$

\begin{tabular}{|c|c|c|}
\hline $\mathcal{O}$ & $\mu(\mathcal{O}, \phi)$ & $\mathcal{U}(\mathcal{O}, \phi)$ \\
\hline$E_{7}$ & $\overline{1} 1_{4}$ & St \\
\hline$E_{7}\left(a_{1}\right)$ & $22_{4}$ & D.S. \\
\hline$E_{7}\left(a_{2}\right)$ & $44_{5}$ & D.S. \\
\hline$E_{7}\left(a_{3}\right)$ & 84 & D.S. \\
\hline$E_{7}\left(a_{3}\right)$ & $1_{2}$ & D.S. \\
\hline$D_{6}$ & $\overline{9_{4}}$ & $\mathcal{S U}\left(A_{1},(1)\right)$ \\
\hline$E_{7}\left(a_{4}\right)$ & $\begin{array}{l}44_{3} \\
2_{2}\end{array}$ & $\begin{array}{l}\text { D.S. } \\
\text { D.S. }\end{array}$ \\
\hline$D_{6}\left(a_{1}\right)$ & $9_{2}$ & $\mathcal{S U}\left(A_{1},(1)\right)$ \\
\hline$D_{5}+A_{1}$ & $8_{2}$ & $\mathcal{S U}\left(A_{1},(1)\right)$ \\
\hline$E_{7}\left(a_{5}\right)$ & $\begin{array}{c}12_{1} \\
6_{2}\end{array}$ & $\begin{array}{l}\text { D.S. } \\
\text { D.S. }\end{array}$ \\
\hline$D_{6}\left(a_{2}\right)$ & $16_{1}$ & $\mathcal{S U}\left(A_{1},(1)\right)$ \\
\hline$A_{5}+A_{1}$ & $66_{1}$ & $\mathcal{S U}\left(A_{1},(1)\right)$ \\
\hline$D_{5}\left(a_{1}\right)+A_{1}$ & $44_{1}$ & $0 \leq \nu \leq 1$ \\
\hline$\left(A_{5}\right)^{\prime \prime}$ & 81 & $\mathcal{S U}\left(G_{2},(1,1)\right)$ \\
\hline$D_{4}+A_{1}$ & $9_{3}$ & $\mathcal{S U}\left(B_{2},\left(1, \frac{3}{2}\right)\right)$ \\
\hline$\overline{A_{3} A_{2} A_{1}}$ & $44_{4}$ & $\mathcal{S U}\left(A_{1},(2)\right)$ \\
\hline$D_{4}\left(a_{1}\right)+A_{1}$ & $\begin{array}{l}9_{1} \\
2\end{array}$ & $\begin{array}{l}\left\{0 \leq \nu_{2}<\nu_{1} \leq 3-\nu_{2}, \nu_{1} \leq \frac{5}{2}\right\} \cup\left\{0 \leq \nu_{2}=\nu_{1} \leq \frac{3}{2}\right\} \\
\left\{0 \leq \nu_{2}<\nu_{1} \leq 3-\nu_{2}, \nu_{1} \leq \frac{5}{2}\right\} \cup\left\{0 \leq \nu_{2}=\nu_{1} \leq \frac{5}{2}\right\}\end{array}$ \\
\hline$A_{3}+2 A_{1}$ & 83 & $\begin{array}{c}\left\{0 \leq \nu_{1} \leq \frac{1}{2}, \nu_{1}+2 \nu_{2} \leq \frac{3}{2}\right\} \cup \\
\left\{0 \leq \nu_{1} \leq \frac{1}{2}, 0 \leq \nu_{2} \leq \frac{3}{2}, 2 \nu_{2}-\nu_{1} \geq \frac{3}{2}\right\}\end{array}$ \\
\hline$\left(A_{3}+A_{1}\right)^{\prime \prime}$ & $44_{2}$ & $\mathcal{S U}\left(B_{3},(1,1)\right) \cup\{(1+\nu, \nu,-1+\nu): 1<\nu<2\}$ \\
\hline$A_{2}+3 A_{1}$ & $1_{3}$ & $\begin{array}{c}\mathcal{S U}\left(G_{2},(2,1)\right) \cup\left\{\nu_{1}+\nu_{2}=1,0<\nu_{2}<1\right\} \cup \\
\left\{\nu_{1}=1,0 \leq \nu_{2} \leq 1\right\} \cup\left\{3 \nu_{1}+\nu_{2}=2, \frac{1}{2}<\nu_{2} \leq 2\right\}\end{array}$ \\
\hline $4 A_{1}$ & $22_{3}$ & $\begin{array}{c}\left\{\nu_{1}+\nu_{2}+\nu_{3} \leq \frac{3}{2}\right\} \cup\left\{\nu_{1}+\nu_{2}-\nu_{3} \geq \frac{3}{2}, \nu_{1}<\frac{3}{2}, \nu_{1}+\nu_{2}<2\right\} \\
\cup\left\{\left(\nu_{1}, \nu_{2}, \frac{3}{2}\right): \nu_{1}+\nu_{2} \leq 3, \nu_{1} \leq \frac{5}{2}\right\} \\
\cup\left\{\left(\nu_{1}, 1+\nu_{2},-1+\nu_{2}\right): 0 \leq \nu_{1} \leq \frac{1}{2}, 0 \leq \nu_{2} \leq \frac{1}{2}\right\} \\
\cup\left\{\left(\nu_{1}, 1+\nu_{2},-1+\nu_{2}\right): \nu_{1}+2 \nu_{2}=\frac{3}{2}, \frac{1}{2} \leq \nu_{1} \leq \frac{3}{2}\right\} \\
\cup\left\{\left(\nu, \frac{3}{2}, \frac{1}{2}\right): 0 \leq \nu \leq \frac{5}{2}\right\} \cup\left\{\left(1+\nu,-1+\nu, \frac{3}{2}\right): 0 \leq \nu<\frac{3}{2}\right\} \\
\cup\left\{(2+\nu, \nu,-2+\nu): 0 \leq \nu<\frac{1}{2}\right\} \cup\left\{\left(\nu, \frac{7}{2}, \frac{3}{2}\right): 0 \leq \nu<\frac{1}{2}\right\} \\
\cup\left\{\left(\frac{5}{2}, \frac{3}{2}, \frac{1}{2}\right)\right\} \cup\left\{\left(\frac{7}{2}, \frac{3}{2}, \frac{1}{2}\right)\right\} \cup\left\{\left(\frac{13}{2}, \frac{7}{2}, \frac{3}{2}\right)\right\}\end{array}$ \\
\hline$\left(3 A_{1}\right)^{\prime \prime}$ & $1_{1}$ & $\mathcal{S U}\left(F_{4},(1,2)\right)$, see Table 4 \\
\hline
\end{tabular}

In the next two sections we will present, in detail, the calculations giving the unitary dual of $\mathbb{H}\left(F_{4},(1,2)\right)$. The discussion is organized by nilpotent orbits.

For the distinguished orbits, there is nothing to do. They parameterize discrete series, and the lowest $W$-types and infinitesimal characters can be read from Tables 2 and 3 . 
5.2. Maximal parabolic cases. First we treat the cases of those nilpotent orbits which, in the Bala-Carter classification, correspond to Levi subalgebras of maximal parabolic subalgebras. In terms of the (classical) Langlands classification, they correspond to the induced modules from discrete series on Levi components of maximal parabolic subalgebras. For any Hecke algebra with arbitrary parameters, the Steinberg module $S t$ is a discrete series. In this case, our calculation is more general, and we record it for general parameter $c$. The classification of discrete series depends in general of the particular value of $c$; for the non-Steinberg discrete series, we restrict to the case $c=2$.

Proposition. The Langlands quotient $\bar{X}(M, S t, \nu)$ is unitary if and only if:

\begin{tabular}{|c|c|c|c|}
\hline Levi $M$ & Nilpotent $\mathcal{O}^{1}$ & Central character $\chi$ & Unitary \\
\hline$C_{3}$ & $D_{6}$ & $\left(\nu, 2 c+\frac{1}{2}, c+\frac{1}{2}, \frac{1}{2}\right)$ & $0 \leq \nu \leq\left|c-\frac{3}{2}\right|$ \\
\hline$B_{3}$ & $D_{5}+A_{1}$ & $\left(\frac{c}{2}+1+\nu,-\frac{c}{2}-1+\nu, c+\frac{1}{2}, \frac{1}{2}\right)$ & $0 \leq \nu \leq\left|\frac{c-3}{2}\right|$ \\
\hline$A_{2}+A_{1}$ & $A_{5}+A_{1}$ & $\left(\frac{1}{4}, c-\frac{1}{4},-\frac{1}{4},-\frac{1}{4}-c\right)+\nu\left(\frac{3}{2}, \frac{1}{2}, \frac{1}{2}, \frac{1}{2}\right)$ & $0 \leq \nu \leq \min \left\{\frac{1}{2},\left|2 c-\frac{3}{2}\right|\right\}$ \\
\hline$A_{2}+\widetilde{A}_{1}$ & $A_{3} A_{2} A_{1}$ & $\left(\frac{1}{2}, \frac{c-1}{2},-\frac{c+1}{2}, \frac{1}{2}\right)+\nu(2,1,1,0)$ & $0 \leq \nu \leq \min \left\{\frac{c}{2},\left|\frac{3 c-2}{2}\right|\right\}$ \\
\hline
\end{tabular}

In addition, we have the following complementary series for $\bar{X}(M, \sigma, \nu)$, where $\sigma \neq S t$ is a discrete series:

\begin{tabular}{|c|c|c|c|}
\hline Levi $M$ & Nilpotent $\mathcal{O}$ & Central character $\chi$ & Unitary \\
\hline$C_{3}$ & $D_{6}\left(a_{1}\right)$ & $\left(\nu, \frac{7}{2}, \frac{3}{2}, \frac{1}{2}\right)$ & $0 \leq \nu \leq \frac{1}{2}$ \\
& $D_{6}\left(a_{2}\right)$ & $\left(\nu, \frac{5}{2}, \frac{3}{2}, \frac{1}{2}\right)$ & $0 \leq \nu \leq \frac{1}{2}$ \\
\hline$B_{3}$ & $D_{5}\left(a_{1}\right)+A_{1}$ & $\left(\frac{3}{2}+\nu,-\frac{3}{2}+\nu, \frac{3}{2}, \frac{1}{2}\right)$ & $0 \leq \nu \leq 1$ \\
\hline
\end{tabular}

Proof. The proof is case by case. We compute the intertwining operators for each standard module restricted to every $W$-type. For the necessary calculations, we constructed matrix realizations (with rational entries) for all irreducible representations of the Weyl group of $F_{4}$, and used the software "Mathematica" to multiply matrices in the intertwining operators.

Note that in order to determine the unitarity of the Langlands quotient, we would not need all these intertwining operators, but the byproduct of this calculation is that we obtain the $W$-structure of various irreducible subquotients which appear in the standard modules at reducibility points, in particular (but most importantly), the $W$-structure of the discrete series. (This is the Jantzen-type filtration from Vo1.)

$\mathbf{D}_{6}$ : The infinitesimal character is $\left(\nu, \frac{9}{2}, \frac{5}{2}, \frac{1}{2}\right)$, the lowest $W$-type is $9_{4}$, and $\mathfrak{z}=A_{1}$. The standard module is induced from the Steinberg representation on the Hecke subalgebra of type $C_{3}$. The $W$-structure is

$$
\left.X\left(D_{6}\right)\right|_{W}=9_{4}+8_{4}+4_{5}+2_{4}+1_{4} .
$$

We calculate the intertwining operators for arbitrary parameter $c$. In that case, the infinitesimal character is $\left(\nu, 2 c+\frac{1}{2}, c+\frac{1}{2}, \frac{1}{2}\right)$.

$$
\begin{aligned}
& 9_{4}: 1 ; 8_{4}: \frac{c-\frac{3}{2}-\nu}{c-\frac{3}{2}+\nu} ; 4_{5}: \frac{c+\frac{3}{2}-\nu}{c+\frac{3}{2}+\nu} ; 2_{4}: \frac{\left(c-\frac{3}{2}-\nu\right)\left(3 c-\frac{1}{2}-\nu\right)}{\left(c-\frac{3}{2}+\nu\right)\left(3 c-\frac{1}{2}+\nu\right)} \\
& 1_{4}: \frac{\left(c+\frac{3}{2}-\nu\right)\left(3 c+\frac{5}{2}-\nu\right)}{\left(c+\frac{3}{2}+\nu\right)\left(3 c+\frac{5}{2}+\nu\right)} .
\end{aligned}
$$

\footnotetext{
${ }^{1}$ When $c=2$.
} 
In particular, when $c=2$, the calculation implies that $\bar{X}\left(D_{6}, \nu\right)$ is unitary if and only if $0 \leq \nu \leq \frac{1}{2}$.

$\mathbf{D}_{\mathbf{6}}\left(\mathbf{a}_{\mathbf{1}}\right)$ : The infinitesimal character is $\left(\nu, \frac{7}{2}, \frac{3}{2}, \frac{1}{2}\right)$, the lowest $W$-type is $9_{2}$, and $\mathfrak{z}=A_{1}$. In terms of Langlands classification, the standard module is induced from a one-dimensional discrete series in $C_{3}$. The $W$-structure is

$$
\left.X\left(D_{6}\left(a_{1}\right)\right)\right|_{W}=\operatorname{Ind}_{W\left(C_{3}\right)}^{W\left(F_{4}\right)}(111 \times 0)=9_{2}+4_{3}+8_{4}+1_{2}+2_{4} .
$$

The intertwining operators in this case give:

$$
\begin{aligned}
& 9_{2}: 1 ; 4_{3}: \frac{\frac{1}{2}-\nu}{\frac{1}{2}+\nu} ; 8_{4}: \frac{\frac{7}{2}-\nu}{\frac{7}{2}+\nu} ; 1_{2}: \frac{\left(\frac{1}{2}-\nu\right)\left(\frac{7}{2}-\nu\right)}{\left(\frac{1}{2}+\nu\right)\left(\frac{7}{2}+\nu\right)} ; \\
& 2_{4}: \frac{\left(\frac{7}{2}-\nu\right)\left(\frac{13}{2}-\nu\right)}{\left(\frac{7}{2}+\nu\right)\left(\frac{13}{2}+\nu\right)} .
\end{aligned}
$$

This indicates that $\bar{X}\left(D_{6}\left(a_{1}\right), \nu\right)$ is unitary if and only if $0 \leq \nu \leq \frac{1}{2}$. At $\nu=\frac{1}{2}$, the standard module decomposes as

$$
\begin{aligned}
& X\left(D_{6}\left(a_{1}\right), \frac{1}{2}\right)=\bar{X}\left(D_{6}\left(a_{1}\right), \frac{1}{2}\right)+X\left(E_{7}\left(a_{4}\right), \phi_{1}\right), \\
& \bar{X}\left(D_{6}\left(a_{1}\right), \frac{1}{2}\right)=9_{2}+8_{4}+2_{4} .
\end{aligned}
$$

$\mathbf{D}_{\mathbf{5}}+\mathbf{A}_{\mathbf{1}}$ : The infinitesimal character is $\left(2+\nu,-2+\nu, \frac{5}{2}, \frac{1}{2}\right)$, the lowest $W$-type is $8_{2}$, and $\mathfrak{z}=A_{1}$. The standard module is induced from the Steinberg representation on the Hecke subalgebra of type $B_{3}$. The $W$-structure is

$$
\left.X\left(D_{5}+A_{1}\right)\right|_{W}=8_{2}+2_{2}+9_{4}+4_{5}+1_{4} .
$$

We calculate the intertwining operators for arbitrary parameter $c$. In that case, the infinitesimal character is $\left(\frac{c}{2}+1+\nu,-\frac{c}{2}-1+\nu, c+\frac{1}{2}, \frac{1}{2}\right)$.

$$
\begin{aligned}
& 8_{2}: 1 ; 2_{2}: \frac{\frac{c-3}{2}+\nu}{\frac{c-3}{2}-\nu} ; 9_{4}: \frac{\frac{3 c-1}{2}-\nu}{\frac{2 c-1}{2}+\nu} ; 4_{5}: \frac{\left(\frac{3 c-1}{2}-\nu\right)\left(\frac{3 c+1}{2}-\nu\right)}{\left(\frac{3 c-1}{2}+\nu\right)\left(\frac{3 c+1}{2}+\nu\right)} ; \\
& 1_{4}: \frac{\left.\left(\frac{3 c-1}{2}-\nu\right)\left(\frac{3 c+1}{2}-\nu\right)\left(\frac{5 c+3}{2}-\nu\right)\right)}{\left(\frac{3 c-1}{2}+\nu\right)\left(\frac{3 c+1}{2}+\nu\right)\left(\frac{5 c+3}{2}+\nu\right)} .
\end{aligned}
$$

In particular, if $c=2, \bar{X}\left(D_{5}+A_{1}, \nu\right)$ is unitary if and only if $0 \leq \nu \leq \frac{1}{2}$. At $\nu=\frac{1}{2}$, the standard module decomposes as

$$
\begin{aligned}
& X\left(D_{5}+A_{1}, \frac{1}{2}\right)=\bar{X}\left(D_{5}+A_{1}, \frac{1}{2}\right)+X\left(E_{7}\left(a_{4}\right), \phi_{2}\right), \\
& \left.\bar{X}\left(D_{5}+A_{1}, \frac{1}{2}\right)\right|_{W}=8_{2}+9_{4}+4_{5}+1_{4} .
\end{aligned}
$$

$\mathbf{D}_{\mathbf{6}}\left(\mathbf{a}_{\mathbf{2}}\right)$ : The infinitesimal character is $\left(\nu, \frac{5}{2}, \frac{3}{2}, \frac{1}{2}\right)$, the lowest $W$-type is $16_{1}$, and $\mathfrak{z}=A_{1}$. In terms of Langlands classification, the standard module is induced from a four-dimensional discrete series on $C_{3}$. The $W$-structure is

$$
\begin{aligned}
\left.X\left(D_{6}\left(a_{2}\right)\right)\right|_{W} & =\operatorname{Ind}_{W\left(C_{3}\right)}^{W\left(F_{4}\right)}(1 \times 11+0 \times 111) \\
& =16_{1}+2_{4}+9_{2}+2 \cdot 9_{4}+2 \cdot 8_{4}+12_{1}+8_{2}+2 \cdot 4_{5}+6_{2}+1_{4} .
\end{aligned}
$$


The intertwining operators are (for the $W$-types with multiplicity greater than 1 , we only give the determinant):

$$
\begin{aligned}
& 16_{1}: 1 ; 12_{1}: \frac{\frac{1}{2}-\nu}{\frac{1}{2}+\nu} ; 6_{2}: \frac{\frac{1}{2}-\nu}{\frac{1}{2}+\nu} ; 2_{4}: \frac{\left(\frac{5}{2}-\nu\right)\left(\frac{9}{2}-\nu\right)}{\left(\frac{5}{2}+\nu\right)\left(\frac{9}{2}+\nu\right)} \\
& 8_{2}: \frac{\left(\frac{1}{2}-\nu\right)\left(\frac{5}{2}-\nu\right)}{\left(\frac{1}{2}+\nu\right)\left(\frac{5}{2}+\nu\right)} ; \\
& 1_{4}: \frac{\left(\frac{1}{2}-\nu\right)\left(\frac{5}{2}-\nu\right)\left(\frac{9}{2}-\nu\right)\left(\frac{11}{2}-\nu\right)}{\left(\frac{1}{2}+\nu\right)\left(\frac{5}{2}+\nu\right)\left(\frac{9}{2}+\nu\right)\left(\frac{11}{2}+\nu\right)} ; 92: \frac{\frac{5}{2}-\nu}{\frac{5}{2}+\nu} \\
& 8_{4}: \operatorname{Det}=\frac{\left(\frac{1}{2}-\nu\right)\left(\frac{5}{2}-\nu\right)\left(\frac{9}{2}-\nu\right)}{\left(\frac{1}{2}+\nu\right)\left(\frac{5}{2}+\nu\right)\left(\frac{9}{2}+\nu\right)} \\
& 4_{5}: \text { Det }=\frac{\left(\frac{1}{2}-\nu\right)^{2}\left(\frac{5}{2}-\nu\right)\left(\frac{9}{2}-\nu\right)\left(\frac{11}{2}-\nu\right)}{\left(\frac{1}{2}+\nu\right)^{2}\left(\frac{5}{2}+\nu\right)\left(\frac{9}{2}+\nu\right)\left(\frac{11}{2}+\nu\right)}
\end{aligned}
$$

It follows that $\bar{X}\left(D_{6}\left(a_{2}\right), \nu\right)$ is unitary if and only if $0 \leq \nu \leq \frac{1}{2}$. At $\nu=\frac{1}{2}$, the standard module decomposes as

$$
\begin{aligned}
& X\left(D_{6}\left(a_{2}\right), \frac{1}{2}\right)=\bar{X}\left(D_{6}\left(a_{2}\right), \frac{1}{2}\right)+X\left(E_{7}\left(a_{5}\right), \phi_{1}\right)+X\left(E_{7}\left(a_{5}\right), \phi_{2}\right), \\
& \left.\bar{X}\left(D_{6}\left(a_{2}\right), \frac{1}{2}\right)\right|_{W}=16_{1}+2_{4}+9_{2}+8_{4}+9_{4} .
\end{aligned}
$$

$\mathbf{A}_{5}+\mathbf{A}_{\mathbf{1}}$ : The infinitesimal character is $\left(\frac{1}{4}, \frac{7}{4},-\frac{1}{4},-\frac{9}{4}\right)+\nu\left(\frac{3}{2}, \frac{1}{2}, \frac{1}{2}, \frac{1}{2}\right)$, the lowest $W$ type is $6_{1}$, and $\mathfrak{z}=A_{1}$. In terms of Langlands classification, the standard module is induced from the Steinberg representation on $\widetilde{A}_{2}+A_{1}$.

$$
\begin{aligned}
& \left.X\left(A_{5}+A_{1}\right)\right|_{W}=\operatorname{Ind}_{W\left(\widetilde{A}_{2}+A_{1}\right)}^{W\left(F_{4}\right)}((111) \otimes(11)) \\
& =6_{1}+16_{1}+2 \cdot 8_{4}+8_{2}+4_{3}+4_{5}+12_{1}+9_{2}+2 \cdot 9_{4}+2_{4}+1_{4} .
\end{aligned}
$$

We compute the intertwining operator for arbitrary parameter $c$. In that case, the infinitesimal character is $\left(\frac{1}{4}, c-\frac{1}{4},-\frac{1}{4},-\frac{1}{4}-c\right)+\nu\left(\frac{3}{2}, \frac{1}{2}, \frac{1}{2}, \frac{1}{2}\right)$.

$$
\begin{aligned}
6_{1}: & 1 ; 16_{1}: \frac{\frac{1}{2}-\nu}{\frac{1}{2}+\nu} ; 8_{2}: \frac{\left(\frac{1}{2}-\nu\right)^{2}\left(\frac{3}{2}-\nu\right)}{\left(\frac{1}{2}+\nu\right)^{2}\left(\frac{3}{2}+\nu\right)} ; 12_{1}: \frac{\left(\frac{1}{2}-\nu\right)^{2}}{\left(\frac{1}{2}+\nu\right)^{2}} ; \\
9_{2}: & \frac{\left(\frac{1}{2}-\nu\right)\left(2 c-\frac{3}{2}-\nu\right)}{\left(\frac{1}{2}+\nu\right)\left(2 c-\frac{3}{2}+\nu\right)} ; \\
4_{3}: & \frac{2 c-\frac{3}{2}-\nu}{2 c-\frac{3}{2}+\nu} ; 2_{4}: \frac{\left(\frac{1}{2}-\nu\right)\left(c+\frac{1}{2}-\nu\right)\left(2 c-\frac{3}{2}-\nu\right)\left(2 c+\frac{1}{2}-\nu\right)}{\left(\frac{1}{2}+\nu\right)\left(c+\frac{1}{2}+\nu\right)\left(2 c-\frac{3}{2}+\nu\right)\left(2 c+\frac{1}{2}+\nu\right)} ; \\
4_{5}: & \frac{\left(\frac{1}{2}-\nu\right)^{2}\left(\frac{3}{2}-\nu\right)\left(c+\frac{1}{2}-\nu\right)\left(2 c+\frac{1}{2}-\nu\right)}{\left(\frac{1}{2}+\nu\right)^{2}\left(\frac{3}{2}+\nu\right)\left(c+\frac{1}{2}+\nu\right)\left(2 c+\frac{1}{2}+\nu\right)} \\
1_{4}: & \frac{\left(\frac{1}{2}-\nu\right)^{2}\left(\frac{3}{2}-\nu\right)\left(c+\frac{1}{2}-\nu\right)\left(2 c+\frac{1}{2}-\nu\right)\left(2 c+\frac{3}{2}-\nu\right)}{\left(\frac{1}{2}+\nu\right)^{2}\left(\frac{3}{2}-\nu\right)\left(c+\frac{1}{2}+\nu\right)\left(2 c+\frac{1}{2}+\nu\right)\left(2 c+\frac{3}{2}+\nu\right)} \\
8_{4}: & \text { Det }=\frac{\left(\frac{1}{2}-\nu\right)^{3}\left(c+\frac{1}{2}-\nu\right)\left(2 c-\frac{3}{2}-\nu\right)\left(2 c+\frac{1}{2}-\nu\right)}{\left(\frac{1}{2}+\nu\right)^{3}\left(c+\frac{1}{2}+\nu\right)\left(2 c-\frac{3}{2}+\nu\right)\left(2 c+\frac{1}{2}+\nu\right)} \\
9_{4}: & \text { Det }=\frac{\left(\frac{1}{2}-\nu\right)^{3}\left(\frac{3}{2}-\nu\right)\left(c+\frac{1}{2}-\nu\right)\left(2 c+\frac{1}{2}-\nu\right)}{\left(\frac{1}{2}+\nu\right)^{3}\left(\frac{3}{2}+\nu\right)\left(c+\frac{1}{2}+\nu\right)\left(2 c+\frac{1}{2}+\nu\right)} .
\end{aligned}
$$


It follows that in the case $c=2, \bar{X}\left(A_{5}+A_{1}, \nu\right)$ is unitary if and only if $0 \leq \nu \leq \frac{1}{2}$. At $\nu=\frac{1}{2}$, the standard module decomposes as

$$
\begin{aligned}
& X\left(A_{5} A_{1}, \frac{1}{2}\right)=\bar{X}\left(A_{5} A_{1}, \frac{1}{2}\right)+\bar{X}\left(D_{6}\left(a_{2}\right), \frac{1}{2}\right)+X\left(E_{7}\left(a_{5}\right), \phi_{1}\right), \\
& \bar{X}\left(A_{5} A_{1}, \frac{1}{2}\right)=6_{1}+4_{3} .
\end{aligned}
$$

$\mathbf{D}_{\mathbf{5}}\left(\mathbf{a}_{\mathbf{1}}\right)+\mathbf{A}_{\mathbf{1}}$ : The infinitesimal character is $\left(\frac{3}{2}+\nu,-\frac{3}{2}+\nu, \frac{3}{2}, \frac{1}{2}\right)$, the lowest $W$-type is $4_{1}$, and $\mathfrak{z}=A_{1}$. In terms of Langlands classification, the standard module is induced from a two-dimensional discrete series on $B_{3}$. The $W$-structure is

$$
\left.X\left(D_{5}\left(a_{1}\right)+A_{1}\right)\right)\left.\right|_{W}=\operatorname{Ind}_{W\left(B_{3}\right)}^{W\left(F_{4}\right)}(0 \times 12)=4_{1}+16_{1}+2_{4}+9_{4}+9_{2}+8_{4} .
$$

The intertwining operators are:

$$
\begin{aligned}
& 4_{1}: 1 ; 16_{1}: \frac{1-\nu}{1+\nu} ; 9_{2}: \frac{(1-\nu)(2-\nu)}{(1+\nu)(2+\nu)} ; 9_{4}: \frac{(1-\nu)(4-\nu)}{(1+\nu)(4+\nu)} \\
& 8_{4}: \frac{(1-\nu)(2-\nu)(4-\nu)}{(1+\nu)(2+\nu)(4+\nu)} ; 2_{4}: \frac{(1-\nu)(2-\nu)(4-\nu)(5-\nu)}{(1+\nu)(2+\nu)(4+\nu)(5+\nu)} .
\end{aligned}
$$

It follows that $\bar{X}\left(D_{5}\left(a_{1}\right)+A_{1}\right)$ is unitary if and only if $0 \leq \nu \leq 1$. At $\nu=1$, the standard module decomposes as

$$
\begin{aligned}
& X\left(D_{5}\left(a_{1}\right)+A_{1}, 1\right)=\bar{X}\left(D_{5}\left(a_{1}\right)+A_{1}, 1\right)+\bar{X}\left(D_{6}\left(a_{2}\right), \frac{1}{2}\right), \\
& \left.\bar{X}\left(D_{5}\left(a_{1}\right)+A_{1}, 1\right)\right|_{W}=4_{1} .
\end{aligned}
$$

$\mathbf{A}_{3}+\mathbf{A}_{2}+\mathbf{A}_{1}:$ The infinitesimal character is $\left(\frac{1}{2}, \frac{1}{2},-\frac{3}{2}, \frac{1}{2}\right)+\nu(2,1,1,0)$, the lowest $W$-type is $4_{4}$, and $\mathfrak{z}=A_{1}$. In terms of Langlands classification, the standard module is induced from the Steinberg representation on $A_{2}+\widetilde{A}_{1}$. The $W$-structure is:

$$
\begin{aligned}
\left.X\left(A_{3}+A_{2}+A_{1}\right)\right|_{W} & =\operatorname{Ind}_{W\left(A_{2}+\widetilde{A}_{1}\right)}^{W((111) \otimes(11))} \\
& =4_{4}+16_{1}+8_{4}+2 \cdot 8_{2}+4_{5}+12_{1}+6_{1}+9_{3}+2 \cdot 9_{4}+2_{2}+1_{4} .
\end{aligned}
$$

We compute the intertwining operators for general parameter $c$. In that case the infinitesimal character is $\left(\frac{1}{2}, \frac{c-1}{2},-\frac{c+1}{2}, \frac{1}{2}\right)+\nu(2,1,1,0)$,

$$
\begin{aligned}
& 4_{4}: 1 ; 9_{3}: \frac{\frac{c}{2}-\nu}{\frac{c}{2}+\nu} ; 6_{1}: \frac{\frac{3 c-2}{2}-\nu}{\frac{3 c-2}{2}+\nu} ; 161: \frac{\left(\frac{c}{2}-\nu\right)\left(\frac{3 c-2}{2}-\nu\right)}{\left(\frac{c}{2}+\nu\right)\left(\frac{3 c-2}{2}+\nu\right)} ; \\
& 1_{1}: \frac{\left(\frac{c}{2}-\nu\right)^{2}\left(\frac{3 c-2}{2}-\nu\right)}{\left(\frac{c}{2}+\nu\right)^{2}\left(\frac{3 c-2}{2}+\nu\right)} ; 8_{4}: \frac{\left(\frac{c}{2}-\nu\right)^{2}\left(\frac{3 c-2}{2}-\nu\right)\left(\frac{3 c}{2}-\nu\right)}{\left(\frac{c}{2}+\nu\right)^{2}\left(\frac{3 c-2}{2}+\nu\right)\left(\frac{3 c}{2}+\nu\right)} ; \\
& 2_{2}: \frac{\left(\frac{c}{2}-\nu\right)\left(\frac{c+1}{2}-\nu\right)\left(\frac{c+2}{2}-\nu\right)}{\left(\frac{c}{2}+\nu\right)\left(\frac{c+1}{2}+\nu\right)\left(\frac{c+2}{2}+\nu\right)} ; \\
& 4_{5}: \frac{\left(\frac{c}{2}-\nu\right)^{2}\left(\frac{c+1}{2}-\nu\right)\left(\frac{c+2}{2}-\nu\right)\left(\frac{3 c-2}{2}-\nu\right)\left(\frac{3 c}{2}-\nu\right)}{\left(\frac{c}{2}+\nu\right)^{2}\left(\frac{c+1}{2}+\nu\right)\left(\frac{c+2}{2}+\nu\right)\left(\frac{3 c-2}{2}+\nu\right)\left(\frac{3 c}{2}+\nu\right)} \\
& 1_{4}: \frac{\left(\frac{c}{2}-\nu\right)^{2}\left(\frac{c+1}{2}-\nu\right)\left(\frac{c+2}{2}-\nu\right)\left(\frac{3 c-2}{2}-\nu\right)\left(\frac{3 c}{2}-\nu\right)\left(\frac{3 c+2}{2}-\nu\right)}{\left(\frac{c}{2}+\nu\right)^{2}\left(\frac{c+1}{2}+\nu\right)\left(\frac{c+2}{2}+\nu\right)\left(\frac{3 c-2}{2}+\nu\right)\left(\frac{3 c}{2}+\nu\right)\left(\frac{3 c+2}{2}+\nu\right)} \\
& 8_{2}: \text { Det }=\frac{\left(\frac{c}{2}-\nu\right)^{3}\left(\frac{c+1}{2}-\nu\right)\left(\frac{c+2}{2}-\nu\right)\left(\frac{3 c-2}{2}-\nu\right)}{\left(\frac{c}{2}+\nu\right)^{3}\left(\frac{c+1}{2}+\nu\right)\left(\frac{c+2}{2}+\nu\right)\left(\frac{3 c-2}{2}+\nu\right)} ; \\
& 9_{4}: \text { Det }=\frac{\left(\frac{c}{2}-\nu\right)^{3}\left(\frac{c+1}{2}-\nu\right)\left(\frac{c+2}{2}-\nu\right)\left(\frac{3 c-2}{2}-\nu\right)^{2}\left(\frac{3 c}{2}-\nu\right)}{\left(\frac{c}{2}+\nu\right)^{3}\left(\frac{c+1}{2}+\nu\right)\left(\frac{c+2}{2}+\nu\right)\left(\frac{3 c-2}{2}+\nu\right)^{2}\left(\frac{3 c}{2}+\nu\right)} .
\end{aligned}
$$


In the case $c=2$, it follows that $\bar{X}\left(A_{3}+A_{2}+A_{1}, \nu\right)$ is unitary if and only if $0 \leq \nu \leq 1$. At $\nu=1$, the standard module decomposes as

$$
\begin{aligned}
& X\left(A_{3} A_{2} A_{1}, 1\right)=\bar{X}\left(A_{3} A_{2} A_{1}, 1\right)+\bar{X}\left(D_{4} A_{1},\left(\frac{3}{2}, \frac{1}{2}\right)\right)+X\left(E_{7}\left(a_{5}\right), \phi_{1}\right) ; \\
& \left.\bar{X}\left(A_{3} A_{2} A_{1}, 1\right)\right|_{W}=4_{4}+61 .
\end{aligned}
$$

Note that $\bar{X}\left(A_{3} A_{2} A_{1}, 1\right)$ is the $I M$-dual of $\bar{X}\left(A_{5} A_{1}, \frac{1}{2}\right)$.

5.3. Matching of intertwining operators. Let $(M, \sigma, \nu)$ be a (Hermitian) Langlands parameter, where $\sigma$ is a discrete series module for $\mathbb{H}_{M}$ (or rather $\mathbb{H}_{M_{0}}$, notation as in section 2.5). We construct a Hecke algebra $\mathbb{H}_{\sigma}$ with possibly unequal parameters, with a Weyl group $W_{\sigma}$. Let $\{e, h, f\}$ be a Lie triple parameterizing the tempered module $\sigma$. Then in all cases which we need to consider except for the case $\{e, h, f\} \subset D_{4}\left(a_{1}\right)+A_{1}$, the root system for $\mathbb{H}_{\sigma}$ and $W_{\sigma}$ is the root system of the reductive Lie algebra $\mathfrak{z}(e, h, f)$ of the centralizer in $\mathfrak{g}$ of $\{e, h, f\}$. In the case $D_{4}\left(a_{1}\right)+A_{1}$, the centralizer is of type $2 A_{1}$, but the root system we consider is of type $B_{2}$. The reason is the presence of a component group $\mathbb{Z} / 2 \mathbb{Z}$ in that case.

First, let us collect the relevant results from the operators calculations in the maximal parabolic case, in the previous section. For the case of $\mathbb{H}\left(F_{4},(1,2)\right)$, each generalized discrete series induced from maximal parabolics has a unique lowest $W$-type, $\mu_{0}$.

Lemma. For every maximal parabolic case $(M, \sigma)$, with lowest $W$-type $\mu_{0}$, there is a $W$-type $\mu_{1}$ such that

(i) the operator $r_{\mu_{1}}(M, \sigma, \nu)$ is identical to the operator $r_{s g n}(\nu)$ for a Hecke algebra of type $A_{1}$, and

(ii) the Langlands quotient $\bar{X}(M, \sigma, \nu)$ is unitary if and only if $r_{\mu_{1}}(M, \sigma, \nu)$ is positive semidefinite.

Proof. The explicit operators and cases are:

\begin{tabular}{|c|c|c|c|c|}
\hline Levi $M$ & $\mathcal{O}$ & $\mu_{0}$ & $\mu_{1}$ & $r_{\mu_{1}}(M, \sigma, \nu)$ \\
\hline$C_{3}$ & $D_{6}$ & $9_{4}$ & $8_{4}$ & $\frac{1 / 2-\nu}{1 / 2+\nu}$ \\
& $D_{6}\left(a_{1}\right)$ & $9_{2}$ & $4_{3}$ & $\frac{1 / 2-\nu}{1 / 2+\nu}$ \\
& $D_{6}\left(a_{2}\right)$ & $16_{1}$ & $12_{1}$ or $6_{2}$ & $\frac{1 / 2-\nu}{1 / 2+\nu}$ \\
\hline$B_{3}$ & $D_{5}+A_{1}$ & $8_{2}$ & $2_{2}$ & $\frac{1 / 2-\nu}{1 / 2+\nu}$ \\
& $D_{5}\left(a_{1}\right)+A_{1}$ & $4_{1}$ & $16_{1}$ & $\frac{1-\nu}{1+\nu}$ \\
\hline$\widetilde{A}_{2}+A_{1}$ & $A_{5}+A_{1}$ & $6_{1}$ & $16_{1}$ & $\frac{1 / 2-\nu}{1 / 2+\nu}$ \\
\hline$A_{2}+\widetilde{A}_{1}$ & $A_{3}+A_{2}+A_{1}$ & $4_{4}$ & $9_{3}$ & $\frac{1-\nu}{1+\nu}$ \\
\hline
\end{tabular}

Definition. Let $\mathbb{H}$ be an arbitrary graded Hecke algebra. For every $(M, \sigma)$ maximal parabolic case, we call $\sigma$-petite types, the following $W$-types which appear in $X(M, \sigma, \nu)$ : the lowest $W$-types, and any $\mu_{1}$ which satisfy the properties from lemma 5.3. Now assume $(M, \sigma)$ is not maximal parabolic. A $W$-type $\mu$ is called $\sigma$-petite, if for every Levi $M^{\prime}$ such that $M \subset M^{\prime}$ is maximal, the restriction of $\mu$ to $W\left(M^{\prime}\right)$ contains only $\sigma$-petite $W\left(M^{\prime}\right)$-types. 
The main idea is summarized in the next statement, which is proposition 5.6 in BC1 (see also section 2.10 in Ci2]). Recall from (1.3.2) that to every $W$-type $\mu$ which appears in $X(M, \sigma, \nu)$ we attach a $W_{\sigma}$-type $\rho(\mu)$.

Proposition. With the notation above, the set of $\sigma$-petite $W$-types $\left\{\mu_{0}, \mu_{1}, \ldots, \mu_{l}\right\}$ in $X(M, \sigma, \nu)$ with the corresponding $W_{\sigma}$-types $\left\{\rho\left(\mu_{0}\right), \rho\left(\mu_{1}\right), \ldots, \rho\left(\mu_{l}\right)\right\}$, has the property that

$$
r_{\mu}(M, \sigma, \nu)=r_{\rho(\mu)}(\nu), \text { for all } \mu \in\left\{\mu_{0}, \ldots, \mu_{l}\right\},
$$

where $r_{\mu}(M, \sigma, \nu)$ is the operator in $\mathbb{H}$ (defined as in (4.1.5)), and $r_{\rho(\mu)}$ is the spherical operator in $\mathbb{H}_{\sigma}$.

The explicit calculations and details will occupy the rest of this section. The matching of intertwining operators from proposition 5.3 is used to rule out nonunitary modules. Ideally, one would like that the remaining modules are all unitary. This is not the case in general, and more (ad-hoc) arguments are needed to prove nonunitarity.

To prove unitarity, we use deformations of unitary irreducible modules (i.e., complementary series methods), calculations of composition factors and the IwahoriMatsumoto involution.

$\left(\mathbf{A}_{\mathbf{5}}\right)^{\prime \prime}$ : The infinitesimal character is $\left(\nu_{2}+\frac{3 \nu_{1}}{2}, 2+\frac{\nu_{2}}{2}, \frac{\nu_{1}}{2},-2+\frac{\nu_{1}}{2}\right)$, the lowest $W$-type is $8_{1}$, and $\mathfrak{z}=G_{2}$. In the Langlands classification, the standard module is induced from the Steinberg representation on $\widetilde{A}_{2}$. The $W$-structure is

$$
\left.X\left(\left(A_{5}\right)^{\prime \prime}\right)\right|_{W}=\operatorname{Ind}_{W\left(\widetilde{A}_{2}\right)}^{W\left(F_{4}\right)}((111)) .
$$

The restrictions of the nearby $W$-types are as follows:

$\begin{array}{llllll}\text { Nilpotent } & \left(A_{5}\right)^{\prime \prime} & A_{5} A_{1} & D_{6}\left(a_{2}\right) & E_{7}\left(a_{5}\right) & E_{7}\left(a_{5}\right) \\ W \text {-type } & 8_{1} & 6_{1} & 16_{1} & 12_{1} & 6_{2} \\ \text { Multiplicity } & 1 & 1 & 2 & 2 & 1 \\ \widetilde{A}_{2} \subset \widetilde{A}_{2}+A_{1} & (2) & (11) & (2),(11) & (2),(11) & (2) \\ \widetilde{A}_{2} \subset C_{3} & 11 \times 1 & 11 \times 1 & 11 \times 1,1 \times 11 & 11 \times 1,1 \times 11 & 1 \times 11 \\ W(\mathfrak{z})=W\left(G_{2}\right) & (1,0) & (1,3)^{\prime \prime} & (2,2) & (2,1) & (1,3)^{\prime}\end{array}$

We need the intertwining operators for the induced module from the Steinberg representation on $A_{2}$ in the Hecke algebra of type $C_{3}$, with parameters $2-2 \Leftarrow 1$. The operators are

$$
11 \times 1: 1, \quad 1 \times 11: \frac{\frac{1}{2}-\nu}{\frac{1}{2}+\nu}, \quad 111 \times 0: \frac{\frac{3}{2}-\nu}{\frac{3}{2}+\nu} .
$$

Therefore, the matching of operators is with the Hecke algebra of type $G_{2}$ with equal parameters, $\mathbb{H}\left(G_{2}\right)$. The nearby $W$-types match all the relevant $W\left(G_{2}\right)$-types, so the unitary set $\mathcal{U}\left(A_{5}^{\prime \prime}\right)$ is included in the spherical unitary dual of $\mathbb{H}\left(G_{2}\right)$. The modules of $\mathbb{H}\left(G_{2}\right)$ are parameterized by nilpotent orbits in the Lie algebra of type $G_{2}$. We analyze the composition series and unitarity of $X\left(A_{5}^{\prime \prime}\right)$ by cases corresponding to these nilpotent orbits.

(1) In $\mathbb{H}\left(G_{2}\right)$, this is the spherical complementary series

$$
\left\{3 \nu_{1}+2 \nu_{2}<1\right\} \cup\left\{3 \nu_{1}+\nu_{2}>1>2 \nu_{1}+\nu_{2}\right\} .
$$

The standard module $X\left(A_{5}^{\prime \prime}\right)$ is irreducible in these regions, and therefore unitary (being unitarily induced and unitary when $\nu_{2}=0$ ). 
$A_{1}$ : The parameters are of the form $\left(\nu_{1}, \nu_{2}\right)=\left(-\frac{1}{2}+\nu, 1\right)$. They are unitary for $0 \leq \nu<\frac{1}{2}$, being endpoints of the complementary series (5.3.1). The decomposition of the standard module is

$$
\begin{aligned}
X\left(A_{5}^{\prime \prime},\left(1,-\frac{1}{2}+\nu\right)\right) & =\bar{X}\left(A_{5}^{\prime \prime},\left(1,-\frac{1}{2}+\nu\right)\right)+X\left(A_{5}+A_{1}, \nu\right), \\
\left.\bar{X}\left(A_{5}^{\prime \prime},\left(1,-\frac{1}{2}+\nu\right)\right)\right|_{W} & =8_{1}+16_{1}+12_{1}+6_{2}+2 \cdot 8_{4}+4_{3}+4_{5}+2 \cdot 9_{2} \\
& +9_{4}+2_{4}+1_{2} .
\end{aligned}
$$

$\widetilde{A}_{1}$ : The parameters are of the form $\left(\nu_{1}, \nu_{2}\right)=\left(1,-\frac{3}{2}+\nu, 1\right)$. They are unitary for $0 \leq \nu<\frac{1}{2}$, being endpoints of the complementary series (5.3.1). The decomposition of the standard module is

$$
\begin{aligned}
X\left(A_{5}^{\prime \prime},\left(-\frac{3}{2}+\nu, 1\right)\right) & =\bar{X}\left(A_{5}^{\prime \prime},\left(-\frac{3}{2}+\nu, 1\right)\right)+X\left(D_{6}\left(a_{2}\right), \nu\right), \\
\left.\bar{X}\left(A_{5}^{\prime \prime},\left(-\frac{3}{2}+\nu, 1\right)\right)\right|_{W} & =8_{1}+6_{1}+16_{1}+12_{1}+2 \cdot 8_{4}+2 \cdot 4_{3}+2 \cdot 9_{2} \\
& +9_{4}+2_{4}+1_{2} .
\end{aligned}
$$

$G_{2}\left(a_{1}\right)$ : This is the parameter $\left(\nu_{1}, \nu_{2}\right)=(0,1)$. It is unitary, being an endpoint of the complementary series (5.3.1). The decomposition of the standard module is

$$
\begin{aligned}
X\left(A_{5}^{\prime \prime},(0,1)\right) & =\bar{X}\left(A_{5}^{\prime \prime},(0,1)\right)+\bar{X}\left(A_{5} A_{1}, \frac{1}{2}\right)+\bar{X}\left(D_{6}\left(a_{2}\right), \frac{1}{2}\right) \\
& +X\left(E_{7}\left(a_{5}\right), \phi_{1}\right)+X\left(E_{7}\left(a_{5}\right), \phi_{2}\right), \\
\left.\bar{X}\left(A_{5}^{\prime \prime},(0,1)\right)\right|_{W} & =8_{1}+12_{1}+8_{4}+9_{2}+1_{2} .
\end{aligned}
$$

$G_{2}$ : This is the parameter $\left(\nu_{1}, \nu_{2}\right)=(1,1)$. In $\mathbb{H}\left(G_{2}\right)$, it is isolated. We compute the $W$-structure of the standard module. In addition to the operators given by the nearby $W$-types, we need to check the operator on $9_{2}$ :

$$
\begin{aligned}
X\left(A_{5}^{\prime \prime},(1,1)\right) & =\bar{X}\left(A_{5}^{\prime \prime},(1,1)\right)+\bar{X}\left(A_{5} A_{1}, \frac{3}{2}\right)+\bar{X}\left(D_{6}\left(a_{2}\right), \frac{5}{2}\right) \\
& +\bar{X}\left(D_{5} A_{1}, \frac{1}{2}\right)+\bar{X}\left(D_{6}\left(a_{1}\right), \frac{1}{2}\right)+X\left(E_{7}\left(a_{4}\right), \phi_{2}\right), \\
\left.\bar{X}\left(A_{5}^{\prime \prime},(1,1)\right)\right|_{W} & =8_{1}+9_{2} .
\end{aligned}
$$

Moreover, the operator on $9_{2}$ is positive at $(1,1)$, so this point is unitary.

In conclusion, the unitary parameter set $\mathcal{U}\left(A_{5}^{\prime \prime}\right)$ is identical with the spherical unitary dual $\mathcal{S U}\left(\mathbb{H}\left(G_{2}\right)\right)$.

$\mathbf{D}_{4}+\mathbf{A}_{1}$ : The infinitesimal character is $\left(\nu_{1}, \nu_{2}, \frac{5}{2}, \frac{1}{2}\right)$, the lowest $W$-type is $9_{3}$, and $\mathfrak{z}=B_{2}$. In Langlands classification, the standard module is induced from the Steinberg representation on $C_{2}$. The $W$-structure is

$$
\left.X\left(D_{4}+A_{1}\right)\right|_{W}=\operatorname{Ind}_{W\left(C_{2}\right)}^{W\left(F_{4}\right)}(0 \times 11) .
$$

The restrictions of the nearby $W$-types are as follows:

$\begin{array}{llllll}\text { Nilpotent } & D_{4}+A_{1} & D_{5}\left(a_{1}\right) A_{1} & D_{6}\left(a_{2}\right) & E_{7}\left(a_{5}\right) & D_{6}\left(a_{1}\right) \\ W \text {-type } & 9_{3} & 4_{1} & 16_{1} & 12_{1}, 6_{2} & 9_{2} \\ \text { Multiplicity } & 1 & 1 & 2 & 1,1 & 1 \\ B_{2} \subset B_{3} & 1 \times 11 & 0 \times 12 & 0 \times 12,1 \times 11 & 1 \times 11 & 0 \times 12 \\ C_{2} \subset C_{3} & 0 \times 12 & 0 \times 12 & 0 \times 12,1 \times 11 & 1 \times 11 & 1 \times 11 \\ W(\mathfrak{z})=W\left(B_{2}\right) & 2 \times 0 & 11 \times 0 & 1 \times 1 & 0 \times 2 & 0 \times 11\end{array}$


We need the intertwining operators for the induced modules from the Steinberg representation of $C_{2}=B_{2}$ in the Hecke algebras of types $B_{3}$, and $C_{3}$, with parameters $1-1 \Rightarrow 2$, respectively $2-2 \Leftarrow 1$.

In $B_{3}(1-1 \Rightarrow 2)$, the infinitesimal character is $(\nu, 3,2)$, and the standard module is the induced from the Steinberg representation on $B_{2}$. The operators are

$$
1 \times 11: 1, \quad 0 \times 12: \frac{1-\nu}{1+\nu}, \quad 0 \times 111: \frac{4-\nu}{4+\nu} .
$$

In $C_{3}(2-2 \Leftarrow 1)$, the infinitesimal character is $\left(\nu, \frac{5}{2}, \frac{1}{2}\right)$, and the standard module is the induced from the Steinberg representation on $C_{2}$. The operators are

$$
0 \times 12: 1, \quad 1 \times 11: \frac{\frac{3}{2}-\nu}{\frac{3}{2}+\nu}, \quad 0 \times 111: \frac{\left(\frac{3}{2}-\nu\right)\left(\frac{9}{2}-\nu\right)}{\left(\frac{3}{2}+\nu\right)\left(\frac{9}{2}+\nu\right)} .
$$

Therefore, the matching of operators is with the Hecke algebra of type $B_{2}$ with parameters $1 \Rightarrow 3 / 2$. The nearby $W$-types match all the $W\left(B_{2}\right)$-representations of this $B_{2}$, therefore, the unitary parameter set $\mathcal{U}\left(D_{4}+A_{1}\right)$ is included in the spherical unitary dual of $\mathbb{H}\left(B_{2}, 1,3 / 2\right)$. The modules of $\mathbb{H}\left(B_{2}, 1,3 / 2\right)$ are parameterized by a cuspidal local system on $\mathfrak{s} p(2) \oplus \mathbb{C}^{2} \subset \mathfrak{s} p(6)$. Specifically, the nilpotent orbits of $\mathfrak{s} p(6)$ which enter in the parameterization are $(6),(42),(411),(222),\left(21^{4}\right)$. We analyze the composition series and unitarity of $X\left(D_{4}+A_{1}\right)$ by cases corresponding to these nilpotent orbits.

$\left(21^{4}\right)$ : In $H\left(B_{2}, 1,3 / 2\right)$, this is the spherical complementary series

$$
\left\{\nu_{1}+\nu_{2}<1\right\} \cup\left\{\nu_{1}-\nu_{2}>1, \nu_{1}<\frac{3}{2}\right\} .
$$

The standard module $X\left(D_{4}+A_{1}\right)$ is irreducible in these regions, and therefore unitary (being unitarily induced and unitary when $\nu_{2}=0$ ).

(222): The parameters are of the form $\left(\nu_{1}, \nu_{2}\right)=\left(\frac{1}{2}+\nu,-\frac{1}{2}+\nu\right)$. They are unitary for $0 \leq \nu<\frac{3}{2}$, being endpoints of the complementary series (5.3.2). The standard module decomposes as follows:

$$
\begin{aligned}
X\left(D_{4} A_{1},\left(\frac{1}{2}+\nu,-\frac{1}{2}+\nu\right)\right) & =\bar{X}\left(D_{4} A_{1},\left(\frac{1}{2}+\nu,-\frac{1}{2}+\nu\right)+X\left(D_{5}\left(a_{1}\right) A_{1}, \nu\right),\right. \\
\bar{X}\left(D_{4} A_{1},\left.\left(\frac{1}{2}+\nu,-\frac{1}{2}+\nu\right)\right|_{W}\right. & =9_{3}+16_{1}+12_{1}+2 \cdot 9_{4}+8_{4}+2 \cdot 8_{2}+6_{2} \\
& +2 \cdot 4_{5}+2_{2}+1_{4} .
\end{aligned}
$$

(411): The parameters are $\left(\nu_{1}, \nu_{2}\right)=\left(\nu, \frac{3}{2}\right)$. They are unitary for $0 \leq \nu \leq$ $\frac{1}{2}$, being endpoints of the complementary series (5.3.2). The standard module decomposes as follows:

$$
\begin{aligned}
X\left(D_{4} A_{1},\left(\nu, \frac{3}{2}\right)\right) & =\bar{X}\left(D_{4} A_{1},\left(\nu, \frac{3}{2}\right)\right)+X\left(D_{6}\left(a_{2}\right), \nu\right), \\
\left.\bar{X}\left(D_{4} A_{1},\left(\nu, \frac{3}{2}\right)\right)\right|_{W} & =9_{3}+16_{1}+8_{2}+4_{1}+2_{2}+9_{4} .
\end{aligned}
$$

(42): The parameters are $\left(\nu_{1}, \nu_{2}\right)=\left(\frac{3}{2}, \frac{1}{2}\right)$. This is an endpoint of the complementary series (5.3.2), therefore unitary. The decomposition of the standard 
module is

$$
\begin{aligned}
X\left(D_{4} A_{1},\left(\frac{3}{2}, \frac{1}{2}\right)\right) & =\bar{X}\left(D_{4} A_{1},\left(\frac{3}{2}, \frac{1}{2}\right)\right)+\bar{X}\left(D_{5}\left(a_{1}\right) A_{1}, 1\right)+\bar{X}\left(D_{6}\left(a_{2}\right), \frac{1}{2}\right) \\
& +X\left(E_{7}\left(a_{5}\right), \phi_{1}\right)+X\left(E_{7}\left(a_{5}\right), \phi_{2}\right), \\
\left.\bar{X}\left(D_{4} A_{1},\left(\frac{3}{2}, \frac{1}{2}\right)\right)\right|_{W} & =9_{3}+16_{1}+8_{2}+9_{4}+2_{2} .
\end{aligned}
$$

(6): The parameters are $\left(\nu_{1}, \nu_{2}\right)=\left(\frac{5}{2}, \frac{3}{2}\right)$. This point is isolated. From the $W$ structure of $\bar{X}\left(D_{4} A_{1},\left(\nu, \frac{3}{2}\right)\right)$, we see that the only $W$-types which can be present in $\bar{X}\left(D_{4} A_{1},\left(\frac{5}{2}, \frac{3}{2}\right)\right)$ are $9_{3}, 16_{1}, 8_{2}, 4_{1}, 2_{2}, 9_{4}$. By virtue of the matching with operators in $\mathbb{H}\left(B_{2}, 1,3 / 2\right)$, we know $16_{1}$ and $4_{1}$ cannot be present. We compute the operators on the remaining ones and find that the $W$-structure is

$$
\bar{X}\left(D_{4} A_{1},\left(\frac{5}{2}, \frac{3}{2}\right)\right)=9_{3}+8_{2} .
$$

Moreover, the operator on $8_{2}$ is positive, so $\bar{X}\left(D_{4} A_{1},\left(\frac{5}{2}, \frac{3}{2}\right)\right)$ is unitary. Note also that $\bar{X}\left(D_{4} A_{1},\left(\frac{5}{2}, \frac{3}{2}\right)\right)$ is the $I M$-dual of $\bar{X}\left(A_{5}^{\prime \prime},(1,1)\right)$.

In conclusion, the unitary set $\mathcal{U}\left(D_{4}+A_{1}\right)$ is identical with $\mathcal{S U}\left(\mathbb{H}\left(B_{2}, 1,3 / 2\right)\right.$.

$\mathbf{D}_{\mathbf{4}}\left(\mathbf{a}_{\mathbf{1}}\right)+\mathbf{A}_{\mathbf{1}}$ : The infinitesimal character is $\left(\nu_{1}, \nu_{2}, \frac{3}{2}, \frac{1}{2}\right)$, the lowest $W$-types are $9_{1}$ and $2_{1}$, and $\mathfrak{z}=2 A_{1}$. The two lowest $W$-types are separate when $\nu_{1}=\nu_{2}$. If $\nu_{1} \neq \nu_{2}$, then the (Langlands classification) standard module is induced from a one-dimensional discrete series on $B_{2}(1 \Rightarrow 2)$. As a $W$-module:

$$
\left.X\left(D_{4}\left(a_{1}\right) A_{1},\left(\nu_{1}, \nu_{2}\right)\right)\right|_{W}=\operatorname{Ind}_{W\left(B_{2}\right)}^{W\left(F_{4}\right)}(0 \times 2), \quad \text { if } \nu_{1} \neq \nu_{2} .
$$

When $\nu_{1}=\nu_{2}=\nu$, there are two standard modules, corresponding to the two lowest $W$-types, induced from two tempered representations of $B_{3}(1-1 \Rightarrow 2)$. As $W$-modules,

$$
\begin{aligned}
\left.X\left(D_{4}\left(a_{1}\right) A_{1},(\nu, \nu), \phi_{1}\right)\right|_{W} & =\operatorname{Ind}_{W\left(B_{3}\right)}^{W\left(F_{4}\right)}(1 \times 2+0 \times 12)=9_{1}+8_{1}+4_{3}+6_{1} \\
& +2 \cdot 9_{2}+2 \cdot 16_{1}+2 \cdot 8_{4}+9_{4}+12_{1}+4_{1}+2_{4}, \\
\left.X\left(D_{4}\left(a_{1}\right) A_{1},(\nu, \nu), \phi_{2}\right)\right|_{W} & =\operatorname{Ind}_{W\left(B_{3}\right)}^{W\left(F_{4}\right)}(0 \times 3)=2_{1}+8_{1}+4_{3}+9_{2}+1_{2} .
\end{aligned}
$$

The restrictions of the nearby $W$-types are:

$\begin{array}{llllll}\text { Nilpotent } & D_{4}\left(a_{1}\right) A_{1} & D_{4}\left(a_{1}\right) A_{1} & A_{5}^{\prime \prime} & A_{3} A_{2} A_{1} & D_{5}\left(a_{1}\right) A_{1} \\ W \text {-type } & 9_{1} & 2_{1} & 8_{1} & 4_{3} & 4_{1} \\ \text { Multiplicity } & 1+0 & 0+1 & 1+1 & 1+1 & 1+0 \\ B_{2} \subset B_{3} & 1 \times 2 & 0 \times 3 & 1 \times 2,0 \times 3 & 1 \times 2,0 \times 3 & 0 \times 12 \\ C_{2} \subset C_{3} & 12 \times 0 & 12 \times 0 & 12 \times 0,11 \times 1 & 11 \times 1,111 \times 0 & 0 \times 12 \\ W\left(B_{2}\right)= & 2 \times 0 & 11 \times 0 & 1 \times 1 & 1 \times 1 & 11 \times 0 \\ W(\mathfrak{z}) \rtimes \mathbb{Z} / 2 & & & & & \end{array}$

The matching of the intertwining operators is with the Hecke algebra $\mathbb{H}\left(B_{2}, 0,5 / 2\right)$. More specifically,

$$
\begin{array}{ll}
9_{1}: & 1 ; \\
2_{1}: & 1 ; \\
8_{1}: & \left(\begin{array}{cc}
\frac{\frac{5}{5}-\nu_{1}}{2}-\nu_{2} & 0 \\
0 & \frac{\frac{5}{2}-\nu_{2}}{\frac{5}{2}+\nu_{2}}
\end{array}\right) .
\end{array}
$$


For the calculations, we need some intertwining operators on modules in the Hecke algebras of type $B_{3}$ and $C_{3}$.

In $\mathbb{H}\left(B_{3}, 1,2\right)$, the infinitesimal character is $(\nu, 2,1)$. There are two lowest $W$ types $1 \times 2$ and $0 \times 3$, which are separate at $\nu=0$ only. When $\nu>0$, the operators are

$$
1 \times 2: 1, \quad 0 \times 3: 1, \quad 0 \times 12: \frac{3-\nu}{3+\nu} .
$$

In $\mathbb{H}\left(C_{3}, 2,1\right)$, the infinitesimal character is $\left(\nu, \frac{3}{2}, \frac{1}{2}\right)$. The lowest $W$-type is $12 \times 0$. The operators are

$$
12 \times 0: 1, \quad 11 \times 1: \frac{\frac{5}{2}-\nu}{\frac{5}{2}+\nu}, \quad 111 \times 0: \frac{\frac{7}{2}-\nu}{\frac{7}{2}+\nu} .
$$

From this calculation, it follows that the reducibility lines for $X\left(D_{4}\left(a_{1}\right)\right.$, $\left.A_{1},\left(\nu_{1}, \nu_{2}\right)\right)$, when $\nu_{1} \neq \nu_{2}$, are $\nu_{1} \pm \nu_{2}=3, \nu_{i}=\frac{5}{2}, \frac{7}{2}, i=1,2$. The operator on $8_{1}$ shows that the unitary set $\mathcal{U}\left(D_{4}\left(a_{1}\right) A_{1}\right)$ is included in $0 \leq \nu_{2} \leq \nu \leq \frac{5}{2}$. However, the line $\nu_{1}+\nu_{2}=3$ cuts this region. The restrictions in the table above imply that the operator on $4_{1}$ is

$$
4_{1}: \frac{\left(3-\left(\nu_{1}+\nu_{2}\right)\right)\left(3-\left(\nu_{1}-\nu_{2}\right)\right)}{\left(3+\left(\nu_{1}+\nu_{2}\right)\right)\left(3+\left(\nu_{1}-\nu_{2}\right)\right)} .
$$

So for $\nu_{1} \neq \nu_{2}, X\left(D_{4}\left(a_{1}\right) A_{1},\left(\nu_{1}, \nu_{2}\right)\right)$ is unitary if and only if

$$
\left\{\nu_{1}+\nu_{2}<3, \nu_{1}<\frac{5}{2}\right\} .
$$

We also note that $\bar{X}\left(D_{4}\left(a_{1}\right) A_{1},\left(\nu, \frac{5}{2}\right)\right), 0 \leq \nu<\frac{1}{2}$ is the $I M$-dual of $\bar{X}\left(D_{4} A_{1},\left(\nu, \frac{3}{2}\right)\right)$, and $\bar{X}\left(D_{4}\left(a_{1}\right) A_{1},\left(\frac{5}{2}, \frac{1}{2}\right)\right)$ is the $I M$-dual of $\bar{X}\left(D_{4} A_{1},\left(\frac{3}{2}, \frac{1}{2}\right)\right)$. On $\nu_{1}+\nu_{2}=3$, we write the parameters as $\left(\frac{3}{2}+\nu,-\frac{3}{2}+\nu\right)$ (which are unitary for $\left.0 \leq \nu \leq 1\right)$. The $W$-structure here is

$$
\begin{aligned}
X\left(D_{4}\left(a_{1}\right) A_{1},\left(\frac{3}{2}+\nu,-\frac{3}{2}+\nu\right)\right) & =\bar{X}\left(D_{4}\left(a_{1}\right) A_{1},\left(\frac{3}{2}+\nu,-\frac{3}{2}+\nu\right)\right) \\
& +X\left(D_{5}\left(a_{1}\right) A_{1}, \nu\right), \\
\left.\bar{X}\left(D_{4}\left(a_{1}\right) A_{1},\left(\frac{3}{2}+\nu,-\frac{3}{2}+\nu\right)\right)\right|_{W} & =9_{1}+2_{1}+2 \cdot 9_{4}+16_{1}+8_{4}+2 \cdot 8_{1} \\
& +2 \cdot 4_{3}+12_{1}+6_{1}+1_{2} .
\end{aligned}
$$

It remains to analyze the case $\nu_{1}=\nu_{2}=\nu$. The calculation on $8_{1}$ can be used to conclude that both Langlands quotients $\bar{X}\left(D_{4}\left(a_{1}\right) A_{1},(\nu, \nu)\right)$ fail to be unitary for $\nu>\frac{5}{2}$. Moreover, the calculation for $4_{1}$ implies that $\bar{X}\left(D_{4}\left(a_{1}\right) A_{1},(\nu, \nu), \phi_{1}\right)$ is not unitary for $\nu>\frac{3}{2}$.

For $\phi_{1}$, the first possible reducibility point is $\nu=\frac{3}{2}$ (this is seen by looking at the $W$-types in $X\left(D_{4}\left(a_{1}\right) A_{1},(\nu, \nu), \phi_{1}\right)$ and their corresponding nilpotent orbits). So it is unitary for $0 \leq \nu \leq \frac{3}{2}$. The decomposition at $\nu=\frac{3}{2}$ is

$$
\begin{aligned}
X\left(D_{4}\left(a_{1}\right) A_{1},\left(\frac{3}{2}, \frac{3}{2}\right), \phi_{1}\right) & =\bar{X}\left(D_{4}\left(a_{1}\right) A_{1},\left(\frac{3}{2}, \frac{3}{2}\right), \phi_{1}\right)+X\left(D_{5}\left(a_{1}\right) A_{1}, 0\right), \\
\left.X\left(D_{4}\left(a_{1}\right) A_{1},\left(\frac{3}{2}, \frac{3}{2}\right), \phi_{1}\right)\right|_{W} & =9_{1}+9_{2}+16_{1}+8_{4}+8_{1}+12_{1}+6_{1}+4_{3} .
\end{aligned}
$$

For $\phi_{2}$, the first possible reducibility point is $\nu=\frac{5}{2}$, so this is unitary if and only if $0 \leq \nu \leq \frac{5}{2}$. Note that $\bar{X}\left(D_{4}\left(a_{1}\right) A_{1},\left(\frac{5}{2}, \frac{5}{2}\right), \phi_{2}\right)$ is the $I M$-dual of $X\left(E_{7}\left(a_{4}\right), \phi_{1}\right)$. As a $\mathrm{W}$-representation, it is just $2_{1}$. 
In conclusion, the unitary set $\mathcal{U}\left(D_{4}\left(a_{1}\right) A_{1}\right)$ is given by:

(1) $\left\{\nu_{1}+\nu_{2} \leq 3, \nu_{1} \leq \frac{5}{2}\right\}$, if $\nu_{1} \neq \nu_{2}$.

(2) $0 \leq \nu \leq \frac{3}{2}$, if $\nu_{1}=\nu_{2}=\nu$ for the $\phi_{1}$-factor.

(3) $0 \leq \nu \leq \frac{5}{2}$, if $\nu_{1}=\nu_{2}=\nu$ for the $\phi_{2}$-factor.

$\mathbf{A}_{\mathbf{3}}+\mathbf{2} \mathbf{A}_{\mathbf{1}}$ : The infinitesimal character is $\left(\nu_{1}, 1+\nu_{2},-1+\nu_{2}, \frac{1}{2}\right)$, the lowest $W$ type $8_{3}$, and $\mathfrak{z}=2 A_{1}$. In terms of Langlands classification, the standard module is induced from the Steinberg representation on the Hecke algebra of type $A_{1}+\widetilde{A}_{1}$. As a $\mathrm{W}$-module,

$$
\left.X\left(A_{3}+2 A_{1}\right)\right|_{W}=\operatorname{Ind}_{W\left(A_{1}+\widetilde{A}_{1}\right)}^{W\left(F_{4}\right)}((11) \otimes(11)) .
$$

The restrictions of nearby $W$-types are:

$\begin{array}{lllllll}\text { Nilpotent } & A_{3}+2 A_{1} & D_{4}\left(a_{1}\right) A_{1} & A_{3} A_{2} A_{1} & A_{5}^{\prime \prime} & D_{4} A_{1} & D_{5}\left(a_{1}\right) A_{1} \\ W \text {-type } & 8_{3} & 9_{1} & 4_{4} & 8_{1} & 9_{3} & 4_{1} \\ \text { Multiplicity } & 1 & 1 & 1 & 1 & 2 & 1 \\ C_{1} \times A_{1} \subset C_{3} & 1 \times 2 & 1 \times 2 & 1 \times 2 & 11 \times 1 & 1 \times 2,0 \times 12 & 0 \times 12 \\ B_{1} \times A_{1} \subset B_{3} & 11 \times 1 & 1 \times 2 & 11 \times 1 & 1 \times 2 & 1 \times 2,2 \times 1 & 0 \times 12 \\ A_{1} \subset A_{2} & (21) & (21) & \left(1^{3}\right) & (21) & (21),\left(1^{3}\right) & (21) \\ \widetilde{A}_{1} \subset \widetilde{A}_{2} & (21) & (21) & \left(1^{3}\right) & (21) & (21),(21) & (21)\end{array}$

We need the following calculations from the Hecke algebras of type $B_{3}$ and $C_{3}$.

In $\mathbb{H}\left(C_{3}, 2,1\right)$, the infinitesimal character is $\left(1+\nu,-1+\nu, \frac{1}{2}\right)$. The standard module is induced from the Steinberg module on $C_{1} \times A_{1}$, and it is reducible at $\nu=\frac{3}{2}, \frac{5}{2}, \frac{7}{2}$. The relevant operators are:

$$
1 \times 2: 1, \quad 11 \times 1: \frac{\frac{5}{2}-\nu}{\frac{5}{2}+\nu}, \quad 0 \times 12: \frac{\frac{3}{2}-\nu}{\frac{3}{2}+\nu} .
$$

In $\mathbb{H}\left(B_{3}, 1,2\right)$, the infinitesimal character is $\left(\frac{1}{2}+\nu,-\frac{1}{2}+\nu, 2\right)$. The standard module is induced from the Steinberg module on $B_{1} \times A_{1}$, and it is reducible at $\nu=\frac{1}{2}, \frac{5}{2}, \frac{7}{2}$. The relevant operators are:

$$
11 \times 1: 1, \quad 1 \times 2: \frac{\frac{1}{2}-\nu}{\frac{1}{2}+\nu}, \quad 1 \times 11: \frac{\frac{5}{2}-\nu}{\frac{5}{2}+\nu} .
$$

The reducibility lines for $X\left(A_{3}+2 A_{1},\left(\nu_{1}, \nu_{2}\right)\right)$ are $\nu_{1}=\frac{1}{2}, \frac{5}{2}, \frac{7}{2}, \nu_{2}=\frac{3}{2}, \frac{5}{2}, \frac{7}{2}$, and $\pm \nu_{1} \pm 2 \nu_{2}=\frac{3}{2}$ and $\pm \nu_{1} \pm \nu_{2}=3$. (The last two types come from the restrictions of the operators to $A_{1} \subset A_{2}$ and $\widetilde{A}_{1} \subset \widetilde{A}_{2}$.)

From the restrictions we that the operators are as follows:

$$
\begin{aligned}
& 8_{3}: \quad 1 \text {; } \\
& 9_{1}: \frac{\frac{1}{2}-\nu_{1}}{\frac{1}{2}+\nu_{1}} \\
& 4_{1}: \frac{\left(\frac{1}{2}-\nu_{1}\right)\left(\frac{5}{2}-\nu_{1}\right)\left(\frac{3}{2}-\nu_{2}\right)}{\left(\frac{1}{2}+\nu_{1}\right)\left(\frac{5}{2}+\nu_{1}\right)\left(\frac{3}{2}+\nu_{2}\right)} ; \\
& 8_{1}: \quad \frac{\left(\frac{1}{2}-\nu_{1}\right)\left(\frac{5}{2}-\nu_{2}\right)}{\left(\frac{1}{2}+\nu_{1}\right)\left(\frac{5}{2}+\nu_{2}\right)} \text {; } \\
& 4_{4}: \quad \frac{\left(\frac{3}{2}-\left(\nu_{1}+2 \nu_{2}\right)\right)\left(\frac{3}{2}-\left(\nu_{1}-2 \nu_{2}\right)\right.}{\left(\frac{3}{2}+\left(\nu_{1}+2 \nu_{2}\right)\right)\left(\frac{3}{2}+\left(\nu_{1}-2 \nu_{2}\right)\right.} ; \\
& 9_{3}: \quad \text { Det }=\frac{\left(\frac{3}{2}-\nu_{2}\right)\left(\frac{5}{2}-\nu_{1}\right)\left(\frac{3}{2}-\left(\nu_{1}+2 \nu_{2}\right)\right)\left(\frac{3}{2}-\left(\nu_{1}-2 \nu_{2}\right)\right.}{\left(\frac{3}{2}+\nu_{2}\right)\left(\frac{5}{2}+\nu_{1}\right)\left(\frac{3}{2}+\left(\nu_{1}+2 \nu_{2}\right)\right)\left(\frac{3}{2}+\left(\nu_{1}-2 \nu_{2}\right)\right.} \text {. }
\end{aligned}
$$

In this case, it is natural to try to match the unitary set $\mathcal{U}\left(A_{3}+2 A_{1}\right)$ with the spherical unitary dual of $A_{1}+A_{1}$ with parameters 1 and 3 . This is just the set 
$\left\{0 \leq \nu_{1} \leq \frac{1}{2}, 0 \leq \nu_{2} \leq \frac{3}{2}\right\}$. The operators above imply that actually the unitary set is

$\mathcal{U}\left(A_{3}+2 A_{1}\right)=\left\{0 \leq \nu_{1} \leq \frac{1}{2}, \nu_{1}+2 \nu_{2} \leq \frac{3}{2}\right\} \cup\left\{0 \leq \nu_{1} \leq \frac{1}{2}, 0 \leq \nu_{2} \leq \frac{3}{2}, 2 \nu_{2}-\nu_{1} \geq \frac{3}{2}\right\}$.

We also record the relevant decompositions and $W$-structure of the standard module. On $\nu_{2}=\frac{3}{2}, 0 \leq \nu_{1}<\frac{1}{2}$, the decomposition is

$$
X\left(A_{3}+2 A_{1},\left(\nu, \frac{3}{2}\right)\right)=\bar{X}\left(A_{3}+2 A_{1},\left(\nu, \frac{3}{2}\right)\right)+X\left(D_{4}+A_{1},\left(\nu, \frac{1}{2}\right)\right) .
$$

Moreover, $\bar{X}\left(A_{3}+2 A_{1},\left(\nu, \frac{3}{2}\right)\right)$ is self $I M$-dual.

On $\nu_{1}=\frac{1}{2}, 0 \leq \nu_{2}<\frac{1}{2}$ and $1<\nu_{2}<\frac{3}{2}$, the decomposition is

$$
X\left(A_{3}+2 A_{1},\left(\frac{1}{2}, \nu\right)\right)=\bar{X}\left(A_{3}+2 A_{1},\left(\frac{1}{2}, \nu\right)\right)+X\left(D_{4}\left(a_{1}\right)+A_{1},(\nu, \nu), \phi_{1}\right) .
$$

The $I M$-dual of $\bar{X}\left(A_{3}+2 A_{1},\left(\frac{1}{2}, \nu\right)\right)$ is a spherical module.

On $2 \nu_{2} \pm \nu_{1}=\frac{3}{2}$, we write the parameter as $\left(\nu_{1}, \nu_{2}\right)=\left(-\frac{1}{2}+2 \nu, \frac{1}{2}+\nu\right)$. Then it is unitary for $0<\nu<\frac{1}{2}$. Here, the decomposition is

$$
\begin{aligned}
X\left(A_{3}\right. & \left.+2 A_{1},\left(-\frac{1}{2}+2 \nu, \frac{1}{2}+\nu\right)\right) \\
= & \bar{X}\left(A_{3}+2 A_{1},\left(-\frac{1}{2}+2 \nu, \frac{1}{2}+\nu\right)\right)+X\left(A_{3}+A_{2}+A_{1}, \nu\right) .
\end{aligned}
$$

The $I M$-dual of $\bar{X}\left(A_{3}+2 A_{1},\left(-\frac{1}{2}+2 \nu, \frac{1}{2}+\nu\right)\right)$ is parameterized by $4 A_{1}$.

At $\left(\frac{1}{2}, \frac{1}{2}\right)$, the decomposition is

$$
\begin{aligned}
X\left(A_{3}+2 A_{1},\left(\frac{1}{2}, \frac{1}{2}\right)\right)= & \bar{X}\left(A_{3}+2 A_{1},\left(\frac{1}{2}, \frac{1}{2}\right)\right)+X\left(D_{4}\left(a_{1}\right) A_{1},\left(\frac{1}{2}, \frac{1}{2}\right), \phi_{1}\right) \\
& +X\left(A_{3} A_{2} A_{1}, 0\right), \\
\left.\bar{X}\left(A_{3}+2 A_{1},\left(\frac{1}{2}, \frac{1}{2}\right)\right)\right|_{W}= & 8_{3}+16_{1}+8_{2}+12_{1}+6_{2}+9_{3}+9_{4}+4_{5} .
\end{aligned}
$$

The similar decomposition (and identical $W$-structure) holds at $(1,1)$.

Finally, at the point $\left(\frac{1}{2}, \frac{3}{2}\right)$, the decomposition is

$$
\begin{aligned}
X\left(A_{3}+2 A_{1},\left(\frac{1}{2}, \frac{3}{2}\right)\right) & =\bar{X}\left(A_{3}+2 A_{1},\left(\frac{1}{2}, \frac{3}{2}\right)\right)+\bar{X}\left(D_{4}\left(a_{1}\right) A_{1},\left(\frac{3}{2}, \frac{3}{2}\right), \phi_{1}\right) \\
& +\bar{X}\left(D_{4} A_{1},\left(\frac{1}{2}, \frac{1}{2}\right)\right)+X\left(D_{5}\left(a_{1}\right) A_{1}, 0\right), \\
\left.\bar{X}\left(A_{3}+2 A_{1},\left(\frac{1}{2}, \frac{3}{2}\right)\right)\right|_{W} & =8_{3}+16_{1}+8_{2}+4_{4}+12_{1}+6_{1}+9_{3}+9_{4} .
\end{aligned}
$$

Note that $\bar{X}\left(A_{3}+2 A_{1},\left(\frac{1}{2}, \frac{3}{2}\right)\right)$ is the $I M$-dual of $\bar{X}\left(D_{4}\left(a_{1}\right) A_{1},\left(\frac{3}{2}, \frac{3}{2}\right), \phi_{1}\right)$.

$\left(\mathbf{A}_{\mathbf{3}}+\mathbf{A}_{\mathbf{1}}\right)^{\prime \prime}$ : The infinitesimal character is $\left(\frac{\nu_{1}+\nu_{2}}{2}, \frac{\nu_{1}-\nu_{2}}{2}, 1+\frac{\nu_{2}}{2},-1+\frac{\nu_{2}}{2}\right)$, the lowest $W$-type is $4_{2}$, and centralizer $B_{3}$. In the Langlands classification, the standard module is induced from the Steinberg representation on $\widetilde{A}_{1}$, so as a $W$-module, it is $\left.X\left(\left(A_{3}+A_{1}\right)^{\prime \prime}\right)\right|_{W}=\operatorname{Ind}_{W\left(\widetilde{A}_{1}\right)}^{W\left(F_{4}\right)}((11))$.

The restrictions of nearby $W$-types are: 


$\begin{array}{llllll}\text { Nilpotent } & \left(A_{3} A_{1}\right)^{\prime \prime} & A_{3} 2 A_{1} & D 4\left(a_{1}\right) A_{1} & D_{4}\left(a_{1}\right) A_{1} & A_{5}^{\prime \prime} \\ W \text {-types } & 4_{2} & 8_{3} & 9_{1} & 2_{1} & 8_{1} \\ \widetilde{A}_{1} \subset \widetilde{A}_{2} & (21) & 2 \cdot(21) & 3 \cdot(21) & (21) & 3 \cdot(21),\left(1^{3}\right) \\ A_{1} & (2) & (2),(11) & 2 \cdot(2),(11) & (2) & 3 \cdot(2),(11) \\ A_{1} \subset C_{2} & 1 \times 1 & 2(1 \times 1) & 2(1 \times 1), 11 \times 0 & 11 \times 0 & 2(1 \times 1), 2(11 \times 0) \\ W(\mathfrak{z}) & 3 \times 0 & 12 \times 0 & 2 \times 1 & 0 \times 3 & 3 \times 0+1 \times 2\end{array}$

We need a calculation from the Hecke algebra of type $C_{2}, 2 \Leftarrow 1$. The infinitesimal character is $(1+\nu,-1+\nu)$, and the standard module is induced from the Steinberg representation on $\widetilde{A}_{1}$. Then the reducibility points are $\nu=\frac{1}{2}, \frac{3}{2}$, and the operators are:

$$
1 \times 1: 1, \quad 11 \times 0: \frac{\frac{1}{2}-\nu}{\frac{1}{2}+\nu} .
$$

It follows that the reducibility hyperplanes for $X\left(\left(A_{3}+A_{1}\right)^{\prime \prime},\left(\nu_{1}, \nu_{2}\right)\right)$ are $\nu_{i}=$ 1,3 (from $A_{1} \subset C_{2}$ ), $\nu_{i} \pm \nu_{j}=1$ (from $\widetilde{A}_{1} \subset \widetilde{A}_{1}+A_{1}$ ), and $\nu_{1} \pm \nu_{2} \pm \nu_{3}=6$ (from $\left.\widetilde{A}_{1} \subset \widetilde{A}_{2}\right)$.

The matching of operators is with the spherical operators for $\mathbb{H}\left(B_{3}\right)$, the Hecke algebra of type $B_{3}$ with equal parameters. From the tables, we see that we can match the operators on all relevant $W\left(B_{3}\right)$-types, except $1 \times 2$. (The operator on $8_{1}$ fails to match it because of the restriction to $\widetilde{A}_{1} \subset \widetilde{A}_{2}$.) The representation $1 \times 2$ in $B_{3}$ is the only one which rules out the interval $\{(1+\nu, \nu,-1+\nu): 1<\nu<2\}$ in the spherical dual. It follows that the unitary set $\mathcal{U}\left(\left(A_{3} A_{1}\right)^{\prime \prime}\right)$ is included in the union $\mathcal{S U}\left(\mathbb{H}\left(B_{3}\right)\right) \cup\{(1+\nu, \nu,-1+\nu): 1<\nu<2\}$. We also note that the extra hyperplanes of reducibility of $X\left(\left(A_{3}+A_{1}\right)^{\prime \prime}\right)$ which do not correspond to $B_{3}$, intersect this union only in the point $(3,2,1)$. We analyze the parameters in $\mathcal{U}\left(\left(A_{3} A_{1}\right)^{\prime \prime}\right)$ partitioned by the nilpotent orbits in type $\mathfrak{s o}(7)$ (that is, in the same way we parameterize the spherical dual of $\left.\mathbb{H}\left(B_{3}\right)\right)$.

$\left(1^{7}\right)$ : The parameters are $\left(\nu_{1}, \nu_{2}, \nu_{3}\right)$; the spherical complementary series for $\mathbb{H}\left(B_{3}\right)$ is

$$
\left\{\nu_{1}+\nu_{2}<1\right\} \cup\left\{\nu_{1}+\nu_{3}>1, \nu_{2}+\nu_{3}<1, \nu_{1}<1\right\} .
$$

In these regions, $X\left(\left(A_{3}+A_{1}\right)^{\prime \prime}\right)$ is irreducible, and it is unitarily induced and unitary for $\nu_{3}=0$, so it is unitary in (5.3.4).

$\left(221^{3}\right)$ : The parameters are $\left(\frac{1}{2}+\nu_{1},-\frac{1}{2}+\nu_{1}, 2 \nu_{2}\right)$, unitary for $\left\{0 \leq \nu_{1}<\frac{1}{2}, 0 \leq\right.$ $\left.\nu_{2}<\frac{1}{2}\right\}$, being endpoints of the complementary series (5.3.4). The decomposition of the standard module in this region is

$$
\begin{aligned}
\left.X\left(\left(A_{3} A_{1}\right)^{\prime \prime}, \frac{1}{2}+\nu_{1},-\frac{1}{2}+\nu_{1}, 2 \nu_{2}\right)\right) & \left.=\bar{X}\left(\left(A_{3} A_{1}\right)^{\prime \prime}, \frac{1}{2}+\nu_{1},-\frac{1}{2}+\nu_{1}, 2 \nu_{2}\right)\right) \\
& +X\left(A_{3}+2 A_{1},\left(\nu_{1}, \nu_{2}\right)\right) .
\end{aligned}
$$

The $I M$-dual of $\left.\bar{X}\left(\left(A_{3} A_{1}\right)^{\prime \prime}, \frac{1}{2}+\nu_{1},-\frac{1}{2}+\nu_{1}, 2 \nu_{2}\right)\right)$ is parameterized by $4 A_{1}$.

$\left(31^{4}\right)$ : The parameters are $\left(\nu_{1}+\nu_{2}, \nu_{1}-\nu_{2}, 1\right)$, unitary for $\left\{\nu_{1}<\frac{1}{2}\right\}$, being endpoints of (5.3.4). The decomposition of the standard module is

$$
\begin{aligned}
X\left(\left(A_{3} A_{1}\right)^{\prime \prime},\left(\nu_{1}+\nu_{2}, \nu_{1}-\nu_{2}, 1\right)\right) & =\bar{X}\left(\left(A_{3} A_{1}\right)^{\prime \prime},\left(\nu_{1}+\nu_{2}, \nu_{1}-\nu_{2}, 1\right)\right) \\
& +X\left(D_{4}\left(a_{1}\right) A_{1},\left(\nu_{1}, \nu_{2}\right)\right) .
\end{aligned}
$$

The $I M$-dual of $\bar{X}\left(\left(A_{3} A_{1}\right)^{\prime \prime},\left(\nu_{1}+\nu_{2}, \nu_{1}-\nu_{2}, 1\right)\right)$ is a spherical module. 
(322): The parameters are $\left(\frac{1}{2}+\nu,-\frac{1}{2}+\nu, 1\right)$, unitary for $0 \leq \nu<\frac{1}{2}$ (again endpoints of (5.3.4). The standard module decomposes as

$$
\begin{aligned}
X\left(\left(A_{3} A_{1}\right)^{\prime \prime},\left(\frac{1}{2}+\nu,-\frac{1}{2}+\nu, 1\right)\right) & =\bar{X}\left(\left(A_{3} A_{1}\right)^{\prime \prime},\left(\frac{1}{2}+\nu,-\frac{1}{2}+\nu, 1\right)\right) \\
& +X\left(A_{3}+2 A_{1},\left(\nu, \frac{1}{2}\right)+X\left(D_{4}\left(a_{1}\right) A_{1},\left(\nu, \frac{1}{2}\right)\right) .\right.
\end{aligned}
$$

Moreover, $\bar{X}\left(\left(A_{3} A_{1}\right)^{\prime \prime},\left(\frac{1}{2}+\nu,-\frac{1}{2}+\nu, 1\right)\right)$ is self $I M$-dual.

(331): The parameters are $(1+\nu, \nu,-1+\nu)$. At $\nu=0$, we have a unitary module, endpoint of (5.3.4). (Note also that $\bar{X}\left(\left(A_{3} A_{1}\right)^{\prime \prime},(1,1,0)\right)$ is the $I M$-dual of $\bar{X}\left(A_{3}+2 A_{1},\left(\frac{1}{2}, \frac{1}{2}\right)\right)$.) In $\mathbb{H}\left(B_{3}\right)$, this is the only such parameter which is unitary. In $F_{4}$ however, by the observations preceding this analysis, we need to also consider the segment $1<\nu<2$. In this interval, the decomposition is

$$
\begin{gathered}
X\left(\left(A_{3}+A_{1}\right)^{\prime \prime},(1+\nu, \nu,-1+\nu)\right)=\bar{X}\left(\left(A_{3}+A_{1}\right)^{\prime \prime},(1+\nu, \nu,-1+\nu)\right) \\
+2 \cdot \bar{X}\left(A_{3}+2 A_{1},\left(-\frac{1}{2}+\nu, \frac{1}{2}+\frac{\nu}{2}\right)\right)+X\left(A_{3} A_{2} A_{1}, \frac{\nu}{2}\right), \\
\left.\bar{X}\left(\left(A_{3}+A_{1}\right)^{\prime \prime},(1+\nu, \nu,-1+\nu)\right)\right|_{W}=4_{2}+9_{1}+2_{1}+6_{2}+2 \cdot 8_{1}+4_{3} \\
+2 \cdot 9_{2}+12_{1}+16_{1}+8_{4}+1_{2} .
\end{gathered}
$$

This segment is isolated, so to prove that it is unitary, we compute explicitly the signatures on all the $W$-types which appear in the restriction of $\bar{X}\left(\left(A_{3}+A_{1}\right)^{\prime \prime},(1+\right.$ $\nu, \nu,-1+\nu))\left.\right|_{W}$. The $I M$-dual of $\bar{X}\left(\left(A_{3}+A_{1}\right)^{\prime \prime},(1+\nu, \nu,-1+\nu)\right)$ is a module parameterized by $A_{2}+3 A_{1}$, and our calculations will be confirmed by the unitarity of those duals.

(511): The parameters are $(\nu, 2,1)$, which are not unitary for $\nu>0$. At $\nu=0$, $\bar{X}\left(\left(A_{3} A_{1}\right)^{\prime \prime},(2,1,0)\right)$ is the $I M$-dual of $\bar{X}\left(A_{3}+2 A_{1},\left(\frac{1}{2}, 1\right)\right)$, and therefore it is unitary.

(7): The parameter is $(3,2,1)$. Then $\bar{X}\left(\left(A_{3}+A_{1}\right)^{\prime \prime},(3,2,1)\right)$ is the $I M$-dual of $X\left(E_{7}\left(a_{5}\right), \phi_{2}\right)$, and therefore it is unitary.

In conclusion, $\mathcal{U}\left(\left(A_{3}+A_{1}\right)^{\prime \prime}\right)=\mathcal{S U}\left(\mathbb{H}\left(B_{3}\right)\right) \sqcup\{(1+\nu, \nu,-1+\nu): 1<\nu<2\}$.

$\mathbf{A}_{2}+\mathbf{3} \mathbf{A}_{1}$ : The infinitesimal character is $\left(\frac{1}{2},-\frac{1}{2},-\frac{1}{2}, \frac{1}{2}\right)+\nu_{1}(2,1,1,0)+$ $\nu_{2}(1,1,0,0)$, the lowest $W$-type is $1_{3}$, and $\mathfrak{z}=G_{2}$. In terms of Langlands classification, the standard module is induced from the Steinberg representation on $A_{2}$, and as a W-module, $X\left(A_{2}+3 A_{1}\right)=\operatorname{Ind}_{W\left(A_{2}\right)}^{W\left(F_{4}\right)}((111))$.

The restrictions of nearby $W$-types are:

$\begin{array}{llllll}\text { Nilpotent } & A_{2} 3 A_{1} & A_{3} 2 A_{1} & A_{3} A_{2} A_{1} & D_{4} A_{1} & A_{5} A_{1} \\ W \text {-type } & 1_{3} & 8_{3} & 4_{4} & 9_{3} & 6_{1} \\ \text { Multiplicity } & 1 & 1 & 2 & 3 & 1 \\ A_{2} \subset B_{3} & 111 \times 0 & 11 \times 1 & 111 \times 0 & 111 \times 0,1 \times 11 & 11 \times 1 \\ & & & 11 \times 1 & 11 \times 1 & \\ A_{2} \subset A_{2}+\widetilde{A}_{1} & (2) & (2) & (2),(11) & 2 \cdot(2),(11) & (11) \\ W(\mathfrak{z}) & (1,0) & (1,3)^{\prime} & (2,1) & (1,0)+(2,2) & (1,6)\end{array}$

We need a calculation in the Hecke algebra $\mathbb{H}\left(B_{3}, 1,2\right)$. The infinitesimal character is $(\nu+1, \nu,-1+\nu)$, and the standard module is induced from the Steinberg representation on $A_{2}$. The reducibility points are $1,2,3$, and the relevant operators are

$$
111 \times 0: 1, \quad 11 \times 1: \frac{1-\nu}{1+\nu}, \quad 1 \times 11: \frac{(1-\nu)(2-\nu)}{(1+\nu)(2+\nu)} .
$$


Then the reducibility lines for $X\left(A_{2}+3 A_{1},\left(\nu_{1}, \nu_{2}\right)\right)$ are $\nu_{1}, \nu_{1}+\nu_{2}, 2 \nu_{1}+\nu_{2}=$ $1,2,3$, and $\nu_{2}, 3 \nu_{1}+\nu_{2}, 3 \nu_{1}+2 \nu_{2}=2$. The matching of operators is with $\mathbb{H}\left(G_{2}, 1,2\right)$, the Hecke algebra of type $G_{2}: 1<\equiv 2$. From the tables, it follows that we are able to match only the operators on $(1,0),(1,3)^{\prime}$, and $(2,1)$ in $G_{2}$. We will also use the operator on $9_{3}$ (which is the closest one to match $(2,2)$ ). The spherical unitary dual of $\mathbb{H}\left(G_{2}, 1,2\right)$ consists of the closed region $\left\{2 \nu_{1}+\nu_{2} \leq 1\right\}$, and the isolated points $\left(\frac{1}{2}, \frac{1}{2}\right)$ and $(1,2)$. The matched operators are sufficient to conclude that, when irreducible, $X\left(A_{2}+3 A_{1},\left(\nu_{1}, \nu_{2}\right)\right)$ is unitary only in the region $2 \nu_{1}+\nu_{2}<1$, but on the lines we need more information.

On the line $\nu_{1}=-1+\nu, \nu_{2}=2$, the decomposition is

$$
\begin{aligned}
X\left(A_{2}+3 A_{1},(-1+\nu, 2)\right)= & \bar{X}\left(A_{2}+3 A_{1},(-1+\nu, 2)\right)+X\left(A_{3}+A_{2}+A_{1}, \nu\right), \\
\left.\bar{X}\left(A_{2}+3 A_{1},(-1+\nu, 2)\right)\right|_{W}= & 1_{3}+8_{3}+4_{4}+6_{2}+16_{1}+2 \cdot 8_{2}+2 \cdot 9_{3} \\
& +12_{1}+9_{4}+2_{2}+4_{5} .
\end{aligned}
$$

To simplify notation, set $X_{1}(\nu)=X\left(A_{2}+3 A_{1},(-1+\nu, 2)\right)$. The matched operators give

$$
8_{3}:-\frac{\left(\frac{1}{2}-\nu\right)(2-\nu)}{\left(\frac{1}{2}+\nu\right)(2+\nu)}, \quad 4_{4}: \frac{2-\nu}{2+\nu},
$$

showing that $X_{1}(\nu)$ can only be unitary for $\frac{1}{2} \leq \nu \leq 2$. The operator on $9_{3}$ has two nonzero eigenvalues with product $-\frac{\left(\frac{1}{2}-\nu\right)(1-\nu)(2-\nu)^{2}(3-\nu)}{\left(\frac{1}{2}+\nu\right)(1+\nu)(2+\nu)^{2}(3+\nu)}$, so the only segment which can be unitary is $\frac{1}{2} \leq \nu \leq 1$. At $\nu=\frac{1}{2}, X_{1}(\nu)$ decomposes further:

$$
\begin{aligned}
& X_{1}\left(\frac{1}{2}\right)=\bar{X}\left(A_{2}+3 A_{1},\left(\frac{1}{2}, \frac{1}{2}\right)\right)+\bar{X}\left(A_{3}+2 A_{1},\left(\frac{1}{2}, \frac{1}{2}\right)\right) \\
& \left.\bar{X}\left(A_{2}+3 A_{1},\left(\frac{1}{2}, \frac{1}{2}\right)\right)\right|_{W}=1_{3}+4_{4}+8_{2}+9_{3}+2_{2} .
\end{aligned}
$$

Since $X\left(A_{2}+3 A_{1},\left(\frac{1}{2}, \frac{1}{2}\right)\right)$ is the $I M$-dual of $X\left(D_{4}\left(a_{1}\right) A_{1}, \phi_{2}\right)$ (which is tempered), it must be unitary. Also the other factor is unitary, because $\bar{X}\left(A_{3}+2 A_{1},\left(\frac{1}{2}, \frac{1}{2}\right)\right)$ is. Since $9_{2}$ appears in both factors, and the operator on it is positive for $\frac{1}{2}<\nu<1$, it follows that $X_{1}(\nu)$ is unitary for $\frac{1}{2}<\nu<1$. Note also that $X_{1}(\nu)$ is the $I M$-dual of $\bar{X}\left(\left(A_{3}+A_{1}\right)^{\prime \prime}, 1+2 \nu, 2 \nu,-1+2 \nu\right)$, which confirms its unitarity.

On the line $\nu_{1}=1, \nu_{2}=-\frac{3}{2}+\nu$, the decomposition is

$$
\begin{aligned}
X\left(A_{2}+3 A_{1},\left(1,-\frac{3}{2}+\nu\right)\right)= & \bar{X}\left(A_{2}+3 A_{1},\left(1,-\frac{3}{2}+\nu\right)\right) \\
& +\bar{X}\left(A_{3}+2 A_{1},\left(\frac{1}{2}, \nu\right)\right), \\
\left.\bar{X}\left(A_{2}+3 A_{1},\left(1,-\frac{3}{2}+\nu\right)\right)\right|_{W}= & 1_{3}+4_{4}+8_{2}+9_{3}+2_{2} .
\end{aligned}
$$

Denote $X_{2}(\nu)=\bar{X}\left(A_{2}+3 A_{1},\left(1,-\frac{3}{2}+\nu\right)\right)$. The intertwining operators calculations give us

$$
4_{4}: \frac{\frac{7}{2}-\nu}{\frac{7}{2}+\nu}, \quad 9_{3}: \frac{\left(\frac{5}{2}-\nu\right)\left(\frac{7}{2}-\nu\right)}{\left(\frac{5}{2}+\nu\right)\left(\frac{7}{2}+\nu\right)} .
$$

So the only interval on which $X_{2}(\nu)$ can be unitary is $0 \leq \nu \leq \frac{5}{2}$. But on this interval, $X_{2}(\nu)$ is the $I M$-dual of $X\left(D_{4}\left(a_{1}\right) A_{1},(\nu, \nu), \phi_{2}\right)$, so it is in fact unitary. Note that at the endpoint, $\nu=\frac{5}{2}, \bar{X}\left(A_{2}+3 A_{1},(1,1)\right)$ is the $I M$-dual of the discrete series $X\left(E_{7}\left(a_{4}\right), \phi_{2}\right)$. 
Finally, at the point $(1,2), \bar{X}\left(A_{2}+3 A_{1},(1,2)\right)$ is the $I M$-dual of the discrete series $X\left(E_{7}\left(a_{3}\right), \phi_{2}\right)$.

In conclusion, the unitary set $\mathcal{U}\left(A_{2}+3 A_{1}\right)$ is the one pictured in the Figure 3 , It is strictly larger than the spherical unitary dual $\mathcal{S U}\left(\mathbb{H}\left(G_{2}, 1,2\right)\right)$.

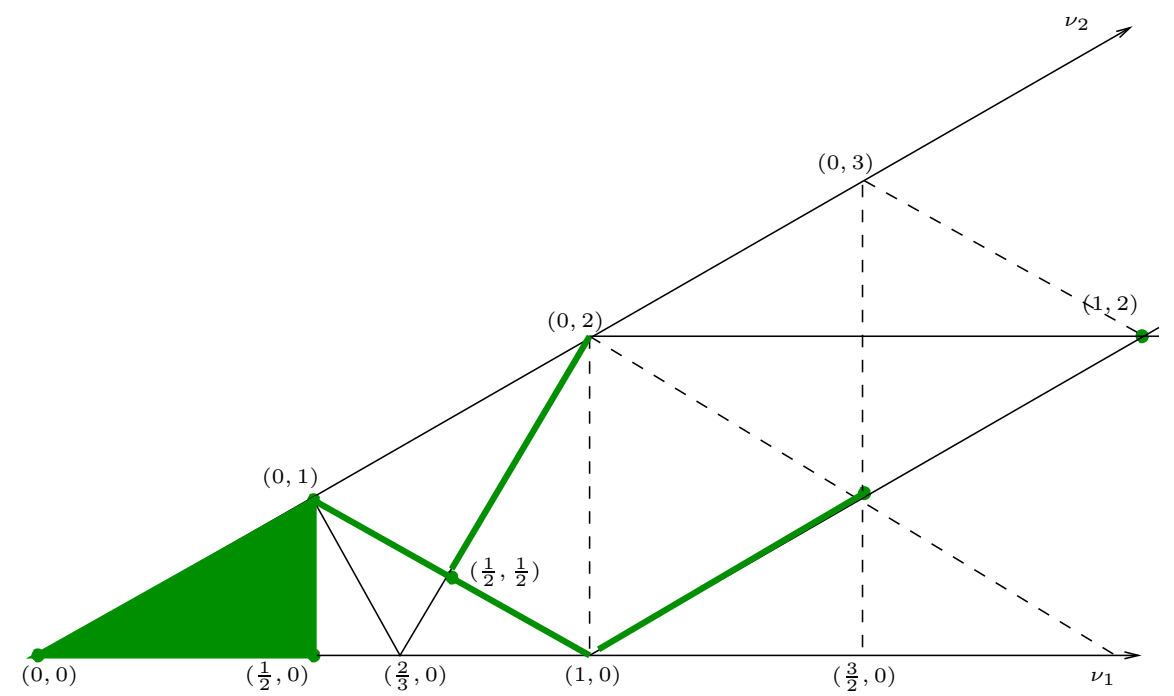

FiguRE 3. Unitary parameters and reducibility lines for $A_{2}+3 A_{1}$

$\mathbf{4 A}_{\mathbf{1}}$ : The parameters are $\left(\nu_{1}, \nu_{2}, \nu_{3}, \frac{1}{2}\right)$, the lowest $W$-type is $2_{3}$, and $\mathfrak{z}=C_{3} . \mathrm{In}$ terms of Langlands classification, the standard module is induced from the Steinberg representation on $A_{1}$, and as a W-representation, $X\left(4 A_{1}\right)=\operatorname{Ind}_{W\left(A_{1}\right)}^{W\left(F_{4}\right)}((11))$.

The restrictions of the nearby $W$-types are:

$\begin{array}{lllllll}\text { Nilpotent } & 4 A_{1} & \left(A_{3} A_{1}\right)^{\prime \prime} & A_{2} 3 A_{1} & A_{3} 2 A_{1} & D_{4}\left(a_{1}\right) A_{1} & A_{3} A_{2} A_{1} \\ W \text {-type } & 23 & 4_{2} & 1_{3} & 8_{3} & 9_{1} & 4_{4} \\ \text { Multiplicity } & 1 & 1 & 1 & 4 & 3 & 3 \\ C_{1} \subset C_{2} & 0 \times 2 & 1 \times 1 & 0 \times 2 & 2(0 \times 2) & 0 \times 2 & 2(0 \times 2) \\ & & & & 2(1 \times 1) & 2(1 \times 1) & 1 \times 1 \\ A_{1} \subset A_{2} & (21) & (21) & \left(1^{3}\right) & 3(21),\left(1^{3}\right) & 3(21) & (21), 2\left(1^{3}\right) \\ \widetilde{A}_{1} & & & & & & \\ W(\mathfrak{z}) & 3 \times 0 & 0 \times 3 & 3 \times 0 & 0 \times 3+2 \times 1 & 1 \times 2 & 2 \times 1\end{array}$

We need one calculation in the Hecke algebra $\mathbb{H}\left(C_{2}, 2,1\right)$ of type $C_{2}, 2 \Leftarrow 1$. The standard module is induced from the Steinberg representation on $C_{1}$, and it has infinitesimal character $\left(\nu, \frac{1}{2}\right)$. The operators are

$$
0 \times 2: 1, \quad 1 \times 1: \frac{\frac{3}{2}-\nu}{\frac{3}{2}+\nu}, \quad 0 \times 11: \frac{\left(\frac{3}{2}-\nu\right)\left(\frac{5}{2}-\nu\right)}{\left(\frac{3}{2}+\nu\right)\left(\frac{5}{2}+\nu\right)} .
$$

The matching of intertwining operators is with the spherical operators for $\mathbb{H}\left(C_{3}, 2,3\right)$, the Hecke algebra of type $C_{3}, 2-2 \Leftarrow 3$. The only $W$-types which give matchings are: 


$$
\begin{array}{ll}
2_{3} & \text { with } 3 \times 0 \\
4_{2} & \text { with } 0 \times 3 \\
9_{1} & \text { with } 1 \times 2 \\
8_{1} & \text { with } 0 \times 12
\end{array}
$$

The last one is irrelevant for the calculation. To get an inclusion of $\mathcal{U}\left(4 A_{1}\right)$ into $\mathcal{S U}\left(\mathbb{H}\left(C_{3}, 2,3\right)\right)$, we would have needed to find matchings for the $W\left(C_{3}\right)$-types $2 \times 1$ and $12 \times 0$ as well. The $W\left(F_{4}\right)$-type $8_{3}$ fails to match $2 \times 1$ because of its restriction to $A_{1} \subset A_{2}$, but we will have to use it in the calculation nevertheless. We will also use the operators on $1_{3}$ and $4_{1}$.

The hyperplanes of reducibility for $X\left(4 A_{1}\right),\left(\nu_{1}, \nu_{2}, \nu_{3}\right)$ are: $\nu_{i} \pm \nu_{j}=2, \nu_{i}=\frac{3}{2}$ (as in $\mathbb{H}\left(C_{3}, 2,3\right)$ ), but also $\nu_{i}=\frac{5}{2}$, and $\nu_{1} \pm \nu_{2} \pm \nu_{3}=\frac{3}{2}$. Especially the second extra family of hyperplanes of reducibility will affect the unitarity in an essential way.

The Hecke algebra $\mathbb{H}\left(C_{3}, 2,3\right)$ arises geometrically from a cuspidal local system on $S L(3)^{3}$ in $\operatorname{Spin}(13)$; its dual is parametrized by a subset of the nilpotent orbits in type $B_{6}$. We organize our analysis of the unitary set $\mathcal{U}\left(4 A_{1}\right)$ by infinitesimal characters corresponding to these orbits.

$\left(2^{6} 1\right)$ : In $\mathbb{H}\left(C_{3}, 2,3\right)$, these are parameters $\left(\nu_{1}, \nu_{2}, \nu_{3}\right)$ in the complement of the hyperplanes $\nu_{i} \pm \nu_{j}=2, \nu_{i}=\frac{3}{2}$. In $\mathbb{H}\left(C_{3}, 2,3\right)$ the unitary regions are:

(i) $\left\{\nu_{1}<\frac{3}{2}, \nu_{1}+\nu_{2}<2\right\}$.

(ii) $\left\{\nu_{1}<\frac{3}{2}, \nu_{1}+\nu_{3}>2, \nu_{2}+\nu_{3}<2\right\}$.

But the operators on the matched $W\left(C_{3}\right)$-types $3 \times 0,0 \times 3,1 \times 2$ (equivalently, on the $W\left(F_{4}\right)$-operators $2_{3}, 4_{2}$, respectively $\left.9_{1}\right)$ are also positive in the region

(iii) $\left\{\nu_{3}<\frac{3}{2}, \nu_{2}>\frac{3}{2}, \nu_{1}+\nu_{3}>2, \nu_{1}-\nu_{3}<2, \nu_{2}+\nu_{3}<2\right\}$.

In $F_{4}, X\left(4 A_{1}\right)$ becomes reducible also on the hyperplanes $\nu_{1} \pm \nu_{2} \pm \nu_{3}=\frac{3}{2}$, some of which cut the regions (i)-(iii). More specifically, region (i) is cut by the hyperplanes $\nu_{1}+\nu_{2}+\nu_{3}=\frac{3}{2}$ and $\nu_{1}+\nu_{2}-\nu_{3}=\frac{3}{2}$, and region (ii) is cut by $\nu_{1}+\nu_{2}-\nu_{3}=\frac{3}{2}$. We use the operators on $1_{2}$ and $8_{4}$ and these are indefinite in all the resulting (sub)regions except

$$
\left\{\nu_{1}+\nu_{2}+\nu_{3} \leq \frac{3}{2}\right\} \cup\left\{\nu_{1}+\nu_{2}-\nu_{3} \geq \frac{3}{2}, \nu_{1}<\frac{3}{2}, \nu_{1}+\nu_{2}<2\right\} .
$$

In these two remaining regions, one can deform $\nu_{3}$ to 0 , and $X\left(4 A_{1}\right)$ stays irreducible, and for $\nu_{3}=0$, it is unitarily induced and unitary. So the parameters in (5.3.6) are unitary. On the hyperplanes $\nu_{1}+\nu_{2} \pm \nu_{3}=\frac{3}{2}$, in (5.3.6), the decomposition is

$$
X\left(4 A_{1}\right)=\bar{X}\left(4 A_{1}\right)+X\left(A_{2}+3 A_{1}\right),
$$

and the factor $\bar{X}\left(4 A_{1}\right)$ is self $I M$-dual.

$\left(52^{4}\right)$ : The parameter is $\left(\nu_{1}, \nu_{2}, \frac{3}{2}\right)$. In $\mathbb{H}\left(C_{3}, 2,3\right)$, the corresponding parameters are unitary for $\left\{\nu_{1}<\frac{1}{2}\right\} \cup\left\{\nu_{2}>\frac{1}{2}, \nu_{1}+\nu<2\right\}$. However, out of the matched operators, the only nontrivial one is on $9_{1}$, and it is $\frac{\left(\frac{7}{2}-\nu_{1}\right)\left(\frac{7}{2}-\nu_{2}\right)}{\left(\frac{7}{2}+\nu_{1}\right)\left(\frac{7}{2}+\nu_{2}\right)}$. We also need the operators on $1_{3}$ and $4_{1}$ :

$$
1_{3}:\left\{\begin{array}{cl}
\frac{\left(3-\left(\nu_{1}-\nu_{2}\right)\right)\left(3-\left(\nu_{1}+\nu_{2}\right)\right)}{\left(3+\left(\nu_{1}-\nu_{2}\right)\right)\left(3+\left(\nu_{1}+\nu_{2}\right)\right)}, & \nu_{1} \neq \nu_{2}, \\
0, & \nu_{1}=\nu_{2},
\end{array} 4_{1}: \frac{\left(\frac{5}{2}-\nu_{1}\right)\left(\frac{5}{2}-\nu_{2}\right)\left(\frac{7}{2}-\nu_{1}\right)\left(\frac{7}{2}-\nu_{2}\right)}{\left(\frac{5}{2}+\nu_{1}\right)\left(\frac{5}{2}+\nu_{2}\right)\left(\frac{7}{2}+\nu_{1}\right)\left(\frac{7}{2}+\nu_{2}\right)} .\right.
$$

From these calculations, it follows that for $\nu_{1} \neq \nu_{2}$, the only unitary parameters are in the region $\left\{\nu_{1}+\nu_{2}<3, \nu_{1}<\frac{5}{2}\right\}$ (and its closure). In this region, the 
decomposition of the standard module is

$$
\begin{aligned}
X\left(4 A_{1},\left(\nu_{1}, \nu_{2}, \frac{3}{2}\right)\right) & =\bar{X}\left(4 A_{1},\left(\nu_{1}, \nu_{2}, \frac{3}{2}\right)\right)+\bar{X}\left(\left(A_{3} A_{1}\right)^{\prime \prime},\left(\nu_{1}+\nu_{2}, \nu_{1}-\nu_{2}, 1\right)\right) \\
& +X\left(D_{4}\left(a_{1}\right) A_{1},\left(\nu_{1}, \nu_{2}\right)\right) .
\end{aligned}
$$

Moreover, $\bar{X}\left(4 A_{1},\left(\nu_{1}, \nu_{2}, \frac{3}{2}\right)\right)$ is the $I M$-dual of $X\left(D_{4}\left(a_{1}\right)+A_{1}\right)$, so it is indeed unitary.

When $\nu_{1}=\nu_{2}=\nu$, the decomposition is

$$
\begin{aligned}
X\left(4 A_{1},\left(\nu, \nu, \frac{3}{2}\right)\right) & =\bar{X}\left(4 A_{1},\left(\nu, \nu, \frac{3}{2}\right)\right)+\bar{X}\left(\left(A_{3} A_{1}\right)^{\prime \prime},(2 \nu, 1,0)\right), \\
& \bar{X}\left(A_{2}+3 A_{1},\left(1,-\frac{3}{2}+\nu\right)\right)+X\left(D_{4}\left(a_{1}\right) A_{1},(\nu, \nu)\right) .
\end{aligned}
$$

The factor $\bar{X}\left(4 A_{1},\left(\nu, \nu, \frac{3}{2}\right)\right)$ is the $I M$-dual of $X\left(D_{4}\left(a_{1}\right) A_{1},(\nu, \nu), p h i_{1}\right)$, so it is unitary if and only if $0 \leq \nu \leq \frac{3}{2}$.

(44221): The parameters are $\left(\nu_{1}, 1+\nu_{2},-1+\nu_{2}\right)$. In $\mathbb{H}\left(C_{3}, 2,3\right)$, they are unitary in the region $\left\{\nu_{1}<\frac{3}{2}, \nu_{2}<\frac{1}{2}\right\}$. In $F_{4}$, the unitary set will be different. Generically, here the standard module decomposes as

$X\left(4 A_{1},\left(\nu_{1}, 1+\nu_{2},-1+\nu_{2}\right)\right)=\bar{X}\left(4 A_{1},\left(\nu_{1}, 1+\nu_{2},-1+\nu_{2}\right)\right)+X\left(A_{3}+2 A_{1},\left(\nu_{1}, \nu_{2}\right)\right)$,

and $\bar{X}\left(4 A_{1}\right)$ is the $I M$-dual of $\bar{X}\left(\left(A_{3}+A_{1}\right)^{\prime \prime},\left(\frac{1}{2}+\nu_{1},-\frac{1}{2}+\nu_{1}, 2 \nu_{2}\right)\right)$, which is unitary for $\left\{0 \leq \nu_{1}<\frac{1}{2}, 0 \leq \nu_{2}<\frac{1}{2}\right\}$.

Similar calculations as in the previous case $\left(\left(52^{4}\right)\right)$ show that the parameters $\left(\nu_{1}, 1+\nu_{2},-1+\nu_{2}\right)$ can be unitary only for $\left\{0 \leq \nu_{1}<\frac{1}{2}, 0 \leq \nu_{2}<\frac{1}{2}\right\}$ (by the remark in the previous paragraph, $\bar{X}\left(4 A_{1},\left(\nu_{1}, 1+\nu_{2},-1+\nu_{2}\right)\right)$ has to be unitary in this region), and on the segment $\nu_{1}+2 \nu_{2}=\frac{3}{2}$, for $\frac{1}{2} \leq \nu_{1} \leq \frac{3}{2}$. For this, we use, in addition to the matched $W$-types, the operators on $1_{3}, 4_{1}, 8_{3}$.

It remains to analyze the segment $\nu_{1}+2 \nu_{2}=\frac{3}{2}$. We rewrite the parameters as $\nu_{1}=\frac{1}{2}+2 \nu, \nu_{2}=\frac{1}{2}-\nu$. Then the factor $\bar{X}\left(4 A_{1},\left(\frac{1}{2}+2 \nu, \frac{1}{2}+\nu,-\frac{3}{2}+\nu\right)\right)$ is the $I M$-dual of $\bar{X}\left(A_{3}+2 A_{1},\left(-\frac{1}{2}+\nu, \frac{1}{2}+\nu\right)\right)$, which is unitary for $0 \leq \nu<\frac{1}{2}$, which is precisely the segment we were looking at. Therefore, this segment is also unitary for $\bar{X}\left(4 A_{1}\right)$.

(53221): The infinitesimal character is $\left(\nu, \frac{3}{2}, \frac{1}{2}\right)$. In $\mathbb{H}\left(C_{3}, 2,3\right)$, the corresponding spherical module is unitary for $0 \leq \nu<\frac{3}{2}$.

In $F_{4}$, the only nonzero matched operator is on $9_{1}$, and it is $\frac{\frac{7}{2}-\nu}{\frac{7}{2}+\nu}$. The operator on $1_{3}$ is $\frac{\left(\frac{5}{2}-\nu\right)\left(\frac{7}{2}-\nu\right)}{\left(\frac{5}{2}+\nu\right)\left(\frac{7}{2}+\nu\right)}$. So it remains to check the segment $0 \leq \nu \leq \frac{5}{2}$. For $0 \leq \nu \leq \frac{5}{2}$, $\bar{X}\left(4 A_{1},\left(\nu, \frac{3}{2}, \frac{1}{2}\right)\right)$ is the $I M$-dual of $X\left(D_{4}\left(a_{1}\right) A_{1},\left(\nu, \frac{1}{2}\right)\right)$, so it is indeed unitary.

(544): The infinitesimal character is $\left(1+\nu,-1+\nu, \frac{3}{2}\right)$. In $\mathbb{H}\left(C_{3}, 2,3\right)$, the corresponding spherical module is unitary for $0 \leq \nu<\frac{3}{2}$. In $F_{4}$, the matched operator on $9_{4}$ gives $\frac{\left(\frac{5}{2}-\nu\right)\left(\frac{9}{2}-\nu\right)}{\left(\frac{5}{2}+\nu\right)\left(\frac{9}{2}+\nu\right)}$. The operator on $1_{2}$ is $\frac{\frac{3}{2}-\nu}{\frac{3}{2}+\nu}$, which implies the only unitary parameters can be in $0 \leq \nu \leq \frac{3}{2}$. For $0 \leq \nu<\frac{3}{2}, \bar{X}\left(4 A_{1},\left(1+\nu,-1+\nu, \frac{3}{2}\right)\right)$ is the $I M$-dual of $X\left(D_{4}\left(a_{1}\right) A_{1},(1+\nu,-1+\nu)\right)$, so it is unitary.

(661): The infinitesimal character is $(2+\nu, \nu,-2+\nu)$. In $\mathbb{H}\left(C_{3}, 2,3\right)$, the corresponding spherical module is unitary for $0 \leq \nu<\frac{1}{2}$. In $F_{4}$, the matched operators give $4_{5}: \frac{\left(\frac{1}{2}+\nu\right)\left(\frac{3}{2}-\nu\right)\left(\frac{7}{2}-\nu\right)}{\left(\frac{1}{2}-\nu\right)\left(\frac{3}{2}+\nu\right)\left(\frac{7}{2}+\nu\right)}$, respectively, $9_{4}: \frac{\left(\frac{3}{2}-\nu\right)\left(\frac{7}{2}-\nu\right)}{\left(\frac{3}{2}+\nu\right)\left(\frac{7}{2}+\nu\right)}$. For $0 \leq \nu<\frac{1}{2}$, the factor 
$\bar{X}\left(4 A_{1},(2+\nu, \nu,-2+\nu)\right)$ is the $I M$-dual of $X\left(\left(A_{5}\right)^{\prime \prime},\left(1,-\frac{1}{2}+\nu\right)\right)$, therefore it is unitary.

(751): The infinitesimal character is $\left(\frac{5}{2}, \frac{3}{2}, \frac{1}{2}\right)$, which is unitary in $\mathbb{H}\left(C_{3}, 2,3\right)$. In $F_{4}, \bar{X}\left(4 A_{1},\left(\frac{5}{2}, \frac{3}{2}, \frac{1}{2}\right)\right)$ is unitary as well, being the $I M$-dual of $\bar{X}\left(D_{6}\left(a_{1}\right), \frac{1}{2}\right)$.

(922): The infinitesimal character is $\left(\nu, \frac{7}{2}, \frac{3}{2}\right)$, which in $\mathbb{H}\left(C_{3}, 2,3\right)$ is unitary for $0 \leq \nu<\frac{1}{2}$. In $F_{4}$, the matched operators are 0 , but the operators on $1_{2}$ and $8_{4}$ are $\frac{\left(\frac{1}{2}+\nu\right)\left(\frac{13}{2}-\nu\right)}{\left(\frac{1}{2}-\nu\right)\left(\frac{13}{2}+\nu\right)}$, respectively $\frac{\frac{13}{2}-\nu}{\frac{13}{2}+\nu}$.

For $0 \leq \nu<\frac{1}{2}$, the factor $\bar{X}\left(4 A_{1},\left(\nu, \frac{7}{2}, \frac{3}{2}\right)\right)$ is the $I M$-dual of $X\left(D_{6}\left(a_{1}\right), \nu\right)$, and therefore unitary; but also the point $\nu=\frac{13}{2}$ is unitary, since $\bar{X}\left(4 A_{1},\left(\frac{13}{2}, \frac{7}{2}, \frac{3}{2}\right)\right)$ is the $I M$-dual of $X\left(E_{7}\left(a_{1}\right)\right)$. (This point has no correspondent in $\mathbb{H}\left(C_{3}, 2,3\right)$.)

(931): The infinitesimal character is $\left(\frac{7}{2}, \frac{3}{2}, \frac{1}{2}\right)$, which is unitary in $\mathbb{H}\left(C_{3}, 2,3\right)$. It is also unitary in $F_{4}$, as $\bar{X}\left(4 A_{1},\left(\frac{7}{2}, \frac{3}{2}, \frac{1}{2}\right)\right)$ is the $I M$-dual of $\bar{X}\left(D_{6}\left(a_{1}\right), \frac{1}{2}\right)$.

(13): The infinitesimal character is $\left(\frac{11}{2}, \frac{7}{2}, \frac{3}{2}\right)$. In $\mathbb{H}\left(C_{3}, 2,3\right)$ the corresponding spherical module is the trivial representation; but $\bar{X}\left(4 A_{1},\left(\frac{11}{2}, \frac{7}{2}, \frac{3}{2}\right)\right)$ is not unitary as seen from the operators for (922) at $\nu=\frac{11}{2}$.

5.4. Spherical modules. The spherical modules are parameterized by the nilpotent orbit $\left(3 A_{1}\right)^{\prime \prime}$. If a spherical module does not contain the sign $W$-representation, via $I M$ its unitarity was already determined in the previous section. We record those results next.

TABLE 4: Spherical unitary modules for $\mathbb{H}\left(F_{4},(1,2)\right)$

\begin{tabular}{|c|c|c|}
\hline Nilpotent & Central character & Unitary \\
\hline$E_{7}$ & $\left(\frac{17}{2}, \frac{9}{2}, \frac{5}{2}, \frac{1}{2}\right)$ & \\
\hline$E_{7}\left(a_{2}\right)$ & $\left(\frac{11}{2}, \frac{5}{2}, \frac{3}{2}, \frac{1}{2}\right)$ & \\
\hline$D_{6}$ & $\left(\nu, \frac{9}{2}, \frac{5}{2}, \frac{1}{2}\right)$ & $\left\{0 \leq \nu \leq \frac{1}{2}\right\}$ \\
\hline$D_{5}+A_{1}$ & $\left(2+\nu,-2+\nu, \frac{5}{2}, \frac{1}{2}\right)$ & $\left\{0 \leq \nu \leq \frac{1}{2}\right\}$ \\
\hline$E_{7}\left(a_{5}\right)$ & $\left(\frac{5}{2}, \frac{3}{2}, \frac{1}{2}, \frac{1}{2}\right)$ & \\
\hline$D_{6}\left(a_{2}\right)$ & $\left(\nu, \frac{5}{2}, \frac{3}{2}, \frac{1}{2}\right)$ & $\left\{0 \leq \nu<\frac{1}{2}\right\}$ \\
\hline$A_{5}+A_{1}$ & $\left(\frac{1}{4}, \frac{7}{4},-\frac{1}{4},-\frac{9}{4}\right)+\nu\left(\frac{3}{2}, \frac{1}{2}, \frac{1}{2}, \frac{1}{2}\right)$ & $\left\{0 \leq \nu<\frac{1}{2}\right\}$ \\
\hline$\left(A_{5}\right)^{\prime \prime}$ & $\left(\nu_{2}+\frac{3 \nu_{1}}{2}+2+\frac{\nu_{1}}{2}, \frac{\nu_{1}}{2},-2+\frac{\nu_{1}}{2}\right)$ & $\begin{array}{c}\left\{3 \nu_{1}+2 \nu_{2}<1\right\} \\
\left\{2 \nu_{1}+\nu_{2}<1<3 \nu_{1}+\nu_{2}\right\}\end{array}$ \\
\hline$D_{4}+A_{1}$ & $\begin{array}{c}\left(\nu_{1}, \nu_{2}, \frac{5}{2}, \frac{1}{2}\right) \\
\left(\frac{1}{2}+\nu,-\frac{1}{2}+\nu, \frac{5}{2}, \frac{1}{2}\right)\end{array}$ & $\begin{array}{c}\left\{\nu_{1}+\nu_{2}<1\right\} \\
\left\{\nu_{1}-\nu_{2}>1, \nu_{1}<\frac{3}{2}\right\} \\
\left\{0 \leq \nu<\frac{3}{2}\right\}\end{array}$ \\
\hline$A_{3} A_{2} A_{1}$ & $\left(\frac{1}{2}, \frac{1}{2},-\frac{3}{2}, \frac{1}{2}\right)+\nu(2,1,1,0)$ & $\{0 \leq \nu<1\}$ \\
\hline$A_{3}+2 A_{1}$ & $\begin{array}{c}\left(\nu_{1}, 1+\nu_{2},-1+\nu_{2}, \frac{1}{2}\right) \\
\left(1+\nu,-1+\nu, \frac{1}{2}, \frac{1}{2}\right)\end{array}$ & $\begin{array}{c}\left\{0 \leq \nu_{1}<\frac{1}{2}, \nu_{1}+2 \nu_{2}<\frac{3}{2}\right\} \\
\left\{0 \leq \nu_{1}<\frac{1}{2}, 0 \leq \nu_{2}<\frac{3}{2}, 2 \nu_{2}-\nu_{1}>\frac{3}{2}\right\} \\
\left\{0 \leq \nu_{1}<\frac{1}{2}\right\} \cup\left\{1<\nu<\frac{3}{2}\right\}\end{array}$ \\
\hline$\left(A_{3}+A_{1}\right) "$ & $\begin{array}{c}\left(\frac{\nu_{1}+\nu_{2}}{2}, \frac{\nu_{1}-\nu_{2}}{2}, 1+\frac{\nu_{3}}{2},-1+\frac{\nu_{3}}{2}\right) \\
\left(\nu_{1}, \nu_{2}, \frac{3}{2}, \frac{1}{2}\right)\end{array}$ & $\begin{array}{c}\left\{0 \leq \nu_{3} \leq \nu_{2} \leq \nu_{1}<1-\nu_{2}\right\} \\
\left\{0 \leq \nu_{3} \leq \nu_{2}<1-\nu_{3}<\nu_{1}<1\right\} \\
\left\{0 \leq \nu_{2} \leq \nu_{1}<\frac{1}{2}\right\}\end{array}$ \\
\hline$A_{2}+3 A_{1}$ & $\begin{array}{c}\left(\frac{1}{2},-\frac{1}{2},-\frac{1}{2}, \frac{1}{2}\right)+\nu_{1}(2,1,1,0) \\
+\nu_{2}(1,1,0,0)\end{array}$ & $\left\{2 \nu_{1}+\nu_{2}<1\right\}$ \\
\hline $4 A_{1}$ & $\left(\nu_{1}, \nu_{2}, \nu_{3}, \frac{1}{2}\right)$ & $\begin{array}{c}\left\{\nu_{1}+\nu_{2}+\nu_{3}<\frac{3}{2}\right\} \\
\left\{\nu_{1}+\nu_{2}-\nu_{3}>\frac{3}{2}, \nu_{1}<\frac{3}{2}, \nu_{1}+\nu_{2}<2\right\}\end{array}$ \\
\hline$\left(3 A_{1}\right)^{\prime \prime}$ & $\left(\nu_{1}, \nu_{2}, \nu_{3}, \nu_{4}\right)$ & regions $\mathcal{F}_{1}-\mathcal{F}_{5}$ in section 5.5 \\
\hline
\end{tabular}


5.5. The 0-complementary series. In this section we determine the unitary irreducible spherical series $X(\nu)$. The parameter $\nu=\left(\nu_{1}, \nu_{2}, \nu_{3}, \nu_{4}\right)$ is assumed in the dominant Weyl chamber $\mathcal{C}$. This is partitioned by the hyperplanes

$$
\left\langle\alpha_{l}, \nu\right\rangle=1, \alpha_{l} \text { long root and }\left\langle\alpha_{s}, \nu\right\rangle=c, \alpha_{s} \text { short root. }
$$

We assume first that $c>1$ is arbitrary. The principal series $X(\nu)$ is reducible precisely when $\nu$ is on one of the hyperplanes in (5.5.1). If $\mathcal{F}$ is an open connected component of the complement of (5.5.1) in $\mathcal{C}$ (we call $\mathcal{F}$ a region), then all the intertwining operators $r_{\sigma}(\nu), \sigma \in \widehat{W}$, are invertible, and therefore have constant signature in $\mathcal{F}$. We say that the region $\mathcal{F}$ is unitary if $X(\nu)$ is unitary for all (equivalently, any) $\nu \in \mathcal{F}$. The walls of any region $\mathcal{F}$ are of the form (5.5.1), or of the form $\langle\alpha, \nu\rangle=0$, for $\alpha \in \Pi$.

Proposition. Consider the half-space $K=\left\{\nu:\left\langle\epsilon_{1}+\epsilon_{2}, \nu\right\rangle<c\right\}$. The unitary regions $\mathcal{F}$ in $\mathcal{C} \cap K$ are:

$\left(\mathcal{F}_{1}\right)\left\{2 \nu_{1}<1, \nu_{1}+\nu_{2}<c\right\}$;

$\left(\mathcal{F}_{2}\right)\left\{\nu_{1}+\nu_{2}+\nu_{3}+\nu_{4}>1, \nu_{1}+\nu_{2}+\nu_{3}-\nu_{4}<1, \nu_{1}+\nu_{2}<c\right\}$;

$\left(\mathcal{F}_{3}\right)\left\{\nu_{1}+\nu_{2}-\nu_{3}+\nu_{4}>1, \nu_{1}-\nu_{2}+\nu_{3}+\nu_{4}<1, \nu_{1}+\nu_{2}-\nu_{3}-\nu_{4}<1, \nu_{1}+\nu_{2}<c\right\}$;

$\left(\mathcal{F}_{4}\right)\left\{\nu_{1}-\nu_{2}+\nu_{3}+\nu_{4}<1,2 \nu_{2}>1, \nu_{1}+\nu_{2}<c\right\}$;

$\left(\mathcal{F}_{5}\right)\left\{2 \nu_{2}>1, \nu_{1}-\nu_{2}+\nu_{3}-\nu_{4}>1,2 \nu_{3}<1, \nu_{1}-\nu_{2}-\nu_{3}+\nu_{4}<1, \nu_{1}+\nu_{2}<c\right\}$;

$\left(\mathcal{F}_{6}\right)\left\{2 \nu_{2}>1, \nu_{1}-\nu_{2}-\nu_{3}-\nu_{4}>1,2 \nu_{3}<1, \nu_{1}+\nu_{2}<c\right\}$;

$\left(\mathcal{F}_{7}\right)\left\{\nu_{1}-\nu_{2}-\nu_{3}-\nu_{4}>1,2 \nu_{4}>1, \nu_{1}+\nu_{2}<c\right\}$.

Proof. The proof is a case by case analysis, which we sketch here.

Note that $\epsilon_{1}+\epsilon_{2}$ is the highest short root of $F_{4}$. The condition that $\mathcal{F}$ be in the half-space $K$ means that the walls of $\mathcal{F}$ can only be of the form $\left\langle\alpha_{l}, \nu\right\rangle=1$, $\langle\alpha, \nu\rangle=0$ (or $\nu_{1}+\nu_{2}=c$ ). From the partial order relation among the (long) roots, we see that there are 19 such regions. A case by case analysis gives that each of these regions has a wall of the form $\langle\alpha, \nu\rangle=0$.

If $\mathcal{F}$ has a wall of the form $\langle\alpha, \nu\rangle=0$, then on this wall, $X(\nu)$ is unitarily induced irreducibly from a principal series $X_{M}\left(\nu^{\prime}\right)$ for a Hecke subalgebra $H_{M}$, with $M$ Levi of type $B_{3}$ or $C_{3}$. In $[\mathrm{BC} 2$, theorems 3.4 and 3.6, the unitary irreducible spherical principal series for the Hecke algebras of type $B_{n} / C_{n}$ with arbitrary unequal parameters are determined, so in particular we know the unitarity of $X_{M}\left(\nu^{\prime}\right)$, and therefore of $X(\nu)$.

One of the results of $\overline{\mathrm{BC} 2}$, when the Hecke algebra $\mathbb{H}$ if of type $B_{n}$ with arbitrary parameter $c$, is that any region $\mathcal{F}$ on which the highest short root is greater than $c$, is necessarily not unitary. The similar statement is false in $F_{4}$, as seen in the following example.

Example. The region $\mathcal{F}_{8}=\left\{\nu_{1}-\nu_{2}-\nu_{3}-\nu_{4}>1,2 \nu_{4}>1, \nu_{1}+\nu_{3}>c, \nu_{1}+\nu_{4}<c\right\}$ is unitary.

Proof. The region $\mathcal{F}_{8}$ has a wall given by $\left\langle\epsilon_{2}-\epsilon_{3}, \nu\right\rangle=0$. We deform the parameter $\nu$ to this wall, i.e. $\nu_{2}=\nu_{3}$. The corresponding module $X(\nu)$ is unitarily induced irreducible from the principal series $X_{B_{3}}\left(\nu^{\prime}\right)$, where $\nu^{\prime}=\left(\nu_{1}^{\prime}, \nu_{2}^{\prime}, \nu_{3}^{\prime}\right)$ satisfy $\nu_{1}^{\prime}-\nu_{2}^{\prime}>$ $1, \nu_{2}^{\prime}-\nu_{3}^{\prime}>1$, and $\nu_{1}^{\prime}<c$. These parameters $\nu^{\prime}$ are unitary in $B_{3} ; c f$. [BC2, theorem 3.6. 
In order to show that the regions $\mathcal{F}_{1}-\mathcal{F}_{8}$ are the only unitary regions, we determined by brute force computer calculations the signatures of $r_{\sigma}(\nu)$, with $\sigma \in$ $\left\{1_{1}, 4_{2}, 9_{1}\right\}$, and $\nu \in \mathcal{F}$, for $\mathcal{F}$ any region in $\mathcal{C}$.

Corollary. In the case of the geometric Hecke algebra $\mathbb{H}\left(F_{4},(1,2)\right)$, the 0 -complementary series is given by the regions $\mathcal{F}_{1}-\mathcal{F}_{5}$ from proposition 5.5 .

(When $c=2$, the regions $\mathcal{F}_{6}-\mathcal{F}_{8}$, in the notation as before, become empty.)

5.6. $W$-structure. We record the $W$-structure of standard modules for $\mathbb{H}\left(F_{4},(1,2)\right)$ as follows from the intertwining operator calculations of previous sections. The correspondence is normalized so that the nilpotent $E_{7}$ corresponds to the sign representation $1_{4}$, and $\left(3 A_{1}\right)^{\prime \prime}$ to the trivial $1_{1}$. For induced representations and labeling of irreducible characters of the Weyl group of $F_{4}$; see for example [Al].

TABLE 5: $W$-structure of standard modules of $\mathbb{H}\left(F_{4},(1,2)\right)$

\begin{tabular}{|c|c|c|}
\hline $\mathcal{O}$ & $\mu(\mathcal{O}, \phi)$ & $W$-structure \\
\hline$E_{7}$ & $1_{4}$ & $1_{4}$ \\
\hline$E_{7}\left(a_{1}\right)$ & $2_{4}$ & $2_{4}$ \\
\hline$E_{7}\left(a_{2}\right)$ & $4_{5}$ & $4_{5}+1_{4}$ \\
\hline$E_{7}\left(a_{3}\right)$ & $8_{4}$ & $1_{2}$ \\
$E_{7}\left(a_{3}\right)$ & $1_{2}$ & $\operatorname{Ind}_{C_{3}}^{F_{4}}(0 \times 111)$ \\
\hline$D_{6}$ & $9_{4}$ & $4_{3}+1_{2}$ \\
\hline$E_{7}\left(a_{4}\right)$ & $4_{3}$ & $2_{2}$ \\
\hline$D_{6}\left(a_{1}\right)$ & $2_{2}$ & $\operatorname{Ind}_{C_{3}}^{F_{4}}(111 \times 0)$ \\
\hline$D_{5}+A_{1}$ & $8_{2}$ & $\operatorname{Ind}_{B_{3}}^{F_{4}}(0 \times 111)$ \\
\hline$E_{7}\left(a_{5}\right)$ & $12_{1}$ & $12_{1}+8_{4}+4_{5}+82+94+14$ \\
& $6_{2}$ & $6_{2}+45$ \\
\hline$D_{6}\left(a_{2}\right)$ & $16_{1}$ & $\operatorname{Ind}_{C_{3}}^{F_{4}}(1 \times 11+0 \times 111)$ \\
\hline$A_{5}+A_{1}$ & $6_{1}$ & $\operatorname{Ind}_{\widetilde{A}_{2}}^{F_{4}}+A_{1}((111) \otimes(11))$ \\
\hline$D_{5}\left(a_{1}\right)+A_{1}$ & $4_{1}$ & $\operatorname{Ind}_{B_{3}}^{F_{4}}(0 \times 12)$ \\
\hline$\left(A_{5}\right)^{\prime \prime}$ & $8_{1}$ & $\operatorname{Ind}_{\widetilde{A}_{2}}^{F_{4}}((111))$ \\
\hline$D_{4}+A_{1}$ & $9_{3}$ & $\operatorname{Ind}_{C_{2}}^{F_{4}}(0 \times 11)$ \\
\hline$A_{3} A_{2} A_{1}$ & $4_{4}$ & $\operatorname{Ind}_{A_{2}}^{F_{4}} \widetilde{A}_{1}((111) \otimes(11))$ \\
\hline$D_{4}\left(a_{1}\right)+A_{1}$ & $9_{1}$ & $\operatorname{Ind}_{B_{3}}^{F_{4}}(1 \times 2+0 \times 12)$ \\
\hline$A_{3}+2 A_{1}$ & $8_{3}$ & $\operatorname{Ind}_{B_{3}}^{F_{4}}(0 \times 3)$ \\
\hline$\left(A_{3}+A_{1}\right)^{\prime \prime}$ & $4_{2}$ & $\operatorname{Ind}_{A_{1}}^{F_{4}} \widetilde{A}_{1}((11) \otimes(11))$ \\
\hline$A_{2}+3 A_{1}$ & $1_{3}$ & $\operatorname{Ind}_{\widetilde{F}_{1}}((11))$ \\
\hline $4 A_{1}$ & $2_{3}$ & $\operatorname{Ind}_{A_{2}}^{F_{4}}((111))$ \\
\hline$\left(3 A_{1}\right)^{\prime \prime}$ & $1_{1}$ & \\
\hline \hline & & $\operatorname{Ind}_{4}((1))$ \\
\hline$F_{4}$ & \\
\hline$F_{4}$ & & \\
\hline & & \\
\hline
\end{tabular}

As mentioned in the introduction, we verified that the dimensions of the discrete series modules above agree with the dimensions obtained from the weight spaces in [Re1]. In [Re1, one uses the realization of this algebra in $E_{8}$, and employs the 
notation of Spa2 for nilpotent orbits, so to compare the two lists, one needs to add an " $A_{1}$ " to our notation of nilpotent orbits.

\section{ACKNOWLEDGMENTS}

The basis for this paper stems from joint work with D. Barbasch. Most of the ideas here can be traced there, and sometimes even before, to the work of Barbasch and Moy. I thank D. Barbasch for sharing his ideas with me.

I thank G. Lusztig for discussions about the generalized Springer correspondence, and P. Trapa for encouraging me to write up these calculations, and for his many helpful suggestions. I also thank M. Solleveld for a very helpful correspondence. This research was supported by the NSF grant FRG-0554278.

\section{REFERENCES}

[Al] D. Alvis Induce/Restrict matrices for exceptional Weyl groups, preprint.

[Ba] D. Barbasch Unitary spherical spectrum for split classical groups, preprint, arXiv: math/0609828.

[BC1] D. Barbasch, D. Ciubotaru, Whittaker unitary dual of affine graded Hecke algebras of type E, preprint, arXiv:0708.4036.

[BC2] - Spherical unitary principal series, Pure Appl. Math. Q. 1 (2005), no. 4, 755-789. MR.2200999 (2006m:22023)

[BM1] D. Barbasch, A. Moy, A unitarity criterion for p-adic groups, Invent. Math. 98, 1989, 19-38. MR:1010153 (90m:22038)

[BM2] Reduction to real infinitesimal character in affine Hecke algebras, J. Amer. Math. Soc. 6(3), 1993, 611-635. MR1186959 (93k:22015)

[BM3] _ Unitary spherical spectrum for p-adic classical groups, Acta Appl. Math. 44, 1996, 1-37. MR 1407038 (98k:22067)

[BM4] - Whittaker models with an Iwahori fixed vector, Representation theory and analysis on homogeneous spaces (New Brunswick, NJ, 1993), 101-105, Contemp. Math., 177, Amer. Math. Soc., Providence, RI, 1994. MR1303602 (95j:22024)

[BS] W. Beynon, N. Spaltenstein, Green functions of finite Chevalley groups of type $E_{n}(n=$ 6,7,8), J. Algebra 88 (1984), 584-614. MR7477534 (85k:20136)

[BW] A. Borel, N. Wallach, Continuous cohomology, discrete subgroups and representations of reductive groups, Annals of Mathematics Studies, vol. 94, Princeton University Press, Princeton, 1980. MR554917 (83c:22018)

[Ca] R. Carter, Finite groups of Lie type, Wiley-Interscience, New York, 1985. MR794307 (87d:20060)

[Ci1] D. Ciubotaru, The unitary $\mathbb{I}$-spherical dual for split p-adic groups of type $F_{4}$, Represent. Theory, vol. 9, 2005, 94-137. MR2123126 (2005k:22022)

[Ci2] Unitary I-spherical representations for split p-adic $E_{6}$, Represent. Theory 10 (2006), 435-480. MR2266699 (2008d:22017)

[Ev] S. Evens, The Langlands classification for graded Hecke algebras, Proc. Amer. Math. Soc. 124 (1996), no. 4, 1285-1290. MR1322921 (96g:22022)

[Ka] S. Kato, An exotic Deligne-Langlands correspondence for symplectic groups, preprint, arXiv:math/0601155.

[KL] D. Kazhdan, G. Lusztig, Proof of the Deligne-Langlands conjecture for Hecke algebras, Invent. Math. 87, 1987, 153-215. MR862716 (88d:11121)

[Kn] A.W. Knapp, Representation theory of semisimple groups. An overview based on examples, Princeton Mathematical Series, 36, Princeton University Press, 1986. MR855239 $(87 \mathrm{j}: 22022)$

[KR] C. Kriloff, A. Ram, Representations of graded Hecke algebras, Represent. Theory 6 (2002), 31-69. MR.1915086 (2003h:20013)

[LS] L. Lambe, B. Srinivasan, A computation of Green functions for some classical groups, Comm. Algebra 18 (1990), no. 10, 3507-3545. MR1063992 (91i:20041)

[Lu1] G. Lusztig, Affine Hecke algebras and their graded version, J. Amer. Math. Soc. 2, 1989, 599-635. MR 991016 (90e:16049) 
[Lu2] - Cuspidal local systems and graded algebras I, Publ. Math. de l'IHES 67, 1988, 145-202. MR 972345 (90e:22029)

[Lu3] - Cuspidal local systems and graded algebras II, Representations of groups (Banff, AB, 1994), Amer. Math. Soc., Providence, 1995, 217-275. MR.1357201 (96m:22038)

[Lu4] _ Cuspidal local systems and graded algebras III, Represent. Theory 6 (2002), 202242. MR:1927954 (2004k:20010)

[Lu5] - Intersection cohomology complexes on a reductive group, Invent. Math. 75 (1984), no. 2, 205-272. MR732546 (86d:20050)

[Lu6] Classification of unipotent representations of simple p-adic groups, Internat. Math. Res. Notices 1995, no. 11, 517-589. MR 1369407 (98b:22034)

[Lu7] - Character sheaves V, Adv. in Math. 61 (1986), 103-155. MR849848(87m:20118c)

[Mu] G. Muić, The unitary dual of p-adic $G_{2}$, Duke Math. J. 90 (1997), no. 3, 465-493. MR:1480543 (98k:22073)

[Op1] E. Opdam, On the spectral decomposition of affine Hecke algebras, J. Inst. Math. Jussieu 3 (2004), no. 4, 531-648. MR2094450(2005i:20008)

[Op2] — A generating function for the trace of the Iwahori-Hecke algebra, Progr. Math., 210, Birkhäuser Boston, 2003, 301-323. MR1985730 (2004f:20013)

[OS] E. Opdam, M. Solleveld, Discrete series characters for affine Hecke algebras and their formal degrees, preprint, arXiv:0804.0026.

[Re1] M. Reeder, Formal degrees and L-packets of unipotent discrete series representations of exceptional p-adic groups, J. Reine Angew. Math. 520 (2000), 37-93. MR1748271 (2001k:22039)

[Re2] Whittaker models and unipotent representations of p-adic groups, Math. Ann. 308, 587-592 (1997). MR1464911 (98h:22022)

[Sh1] T. Shoji, On the Green polynomials of classical groups, Invent. Math. 74 (1983), 239-267. MR723216 (85f:20032)

[Sh2] On the Green polynomials of a Chevalley group of type $F_{4}$, Commun. Algebra 10 (1982), 505-543. MR647835 (83d:20030)

[Spa1] N. Spaltenstein, On the generalized Springer correspondence for exceptional groups, Algebraic groups and related topics (Kyoto/Nagoya, 1983), 317-338, Adv. Stud. Pure Math., 6, North-Holland, Amsterdam, 1985. MR803340 (87g:20072b)

[Spa2] — Classes unipotentes et sous-groupes de Borel, Lecture Notes in Mathematics, 946, Springer-Verlag, Berlin, New York, 1982. MR672610(84a:14024)

[Spr1] T.A. Springer, A construction of representations of Weyl groups, Invent. Math. 44 (1978), no. 3, 279-293. MR0491988(58:11154)

[Spr2] — Trigonometric sums, Green functions of finite groups and representations of Weyl groups, Invent. Math. 36 (1976), 173-207. MR0442103 (56:491)

[Ta] M. Tadić, Classification of unitary representations in irreducible representations of general linear group (non-Archimedean case), Ann. Sci. École Norm. Sup. (4) 19 (1986), no. 3, 335382. MR 870688 (88b:22021)

[Ti] J. Tits, Reductive groups over local fields, Proceedings of Symposia in Pure Math., vol. 33 (1979), 29-69. MR546588(80h:20064)

[Vo1] D. A. Vogan, Jr., Unitarizability of certain series of representations, Ann. of Math. (2) 120 (1984), no. 1, 141-187. MR750719 (86h:22028)

[Vo2] - Representations of real reductive Lie groups, Progress in Mathematics, 15, Birkhäuser, Boston, MA, 1981. MR632407 (83c:22022)

Department of Mathematics, University of Utah, Salt Lake City, Utah 84112

E-mail address: ciubo@math.utah.edu 